Bo Hyung Cho

Dissertation submitted to the Faculty of the Virginia

Polytechnic Institute and State University in partial

fulfillment of the requirements for the degree of

DOCTOR OF PHILOSOPHY

in

ELECTRICAL ENGINEERING

APPROVED:

F. C. Lee!' Chairman

S. L. Hendricks

S. Rahman

R. H. VanLándingham

V. Vorperian

October, 1985

Blackburg, Virginia 


\section{MODELING AND ANALYSIS OF SPACECRAFT POWER SYSTEMS}

by

Bo Hyung Cho

\section{(ABSTRACT)}

A comprehensive large-scale power system modeling is developed to facilitate the design and analysis of present and future spacecraft power systems. A two-port coupling method is utilized to provide a modularity in model building and analysis of the system. The modular approach allows the model to be flexible, verifiable and computationally efficient. A methodology for the system level analysis is presented with the ability to focus on the performance characteristics of an arbitrary component or subsystem. The system performance parameters are derived explicitly in terms of the two-port hybrid g-parameter representation of the component or subsystem, and impedances of its terminating subsystems. From this, the stability of the system is analytically determined and the subsystem interaction criteria is observed. Also presented is a model development from the empirical data employing the complex curve fitting technique. The technique is especially powerful for large scale system modeling and analysis where certain components and subsystems are viewed as black boxes with measurable terminal characteristics. The technique can also be used to realize a reduced order model of a complex subsystem.

The Direct Energy Transfer (DET) spacecraft power system is modeled to demonstrate the versatility of the comprehensive system model by per- 
forming various DC, small-signal and large-signal analyses. Of particular interest is the analysis of the large-signal behavior of the nonlinear solar array system by employing the state-plane method. The analysis of the solar array system operation focused on the transition mode between the shunt mode and the battery discharging mode is presented. The subsystem interaction problems in the local component and global system are illustrated. A methodology for the design and trouble-shooting of a system dealing with the interaction problems using the g-parameters is described. Finally, a system level analysis of the DET system using an empirical data modeling technique is performed. 


\section{ACKNOWLEDGEMENTS}

I wish to express my deepest gratitude to my advisor, Professor F. C. Lee for his continuing encouragement and guidance throughout the course of this research work. He has cultivated an intellectually stimulating environment in the Power Electronics Research Group conducive to the progress of research.

I also appreciate the support received from entire Power Electronics Research Group who made my stay a very productive and enjoyable experience.

I gratefully acknowledge Naval Research laboratory and NASA Goddard Space Flight Center who sponsored this research work.

My thanks to friends who helped me in typing and proofreading this dissertation.

I thank my wife , who has been a constant source of love and attention. Without her understanding and encouragement, this work would not have been possible.

Finally, and most importantly, I would like to thank my parents. Throughout my life their support and encouragement have never wavered. This debt is truly unpayable, and it is to them that this dissertation is dedicated. 
$\begin{array}{lll}\text { CHAPTER } 1 & \text { INTRODUCTION } & 1\end{array}$

CHAPTER 2 METHODOLOGY OF MODELING AND ANALYSIS OF SPACECRAFT POWER SYSTEM

2.1 Introduction

2.2 Modeling approaches of large scale systems 7

2.3 Two-port transfer function models 12

2.4 System analysis approaches using the transfer $\begin{array}{ll}\text { function models } & 16\end{array}$

2.5 Conclusions 20

CHAPTER 3 SPACECRAFT COMPONENTS MODELING AND ANALYSIS 23

3.1 Introduction 23

3.2 Switching regulator modeling 25

3.3 Solar array modeling 58

3.4 Shunt regulator modeling $\quad 68$

3.5 Cable/filter modeling $\quad 74$

3.6 Payload modeling 76

$\begin{array}{lll}3.7 \text { Conclusions } & 77\end{array}$

CHAPTER 4 MODELING A DIRECT ENERGY TRANSFER SPACECRAFT $\begin{array}{ll}\text { POWER SYSTEM WITH EASY5 } & 78\end{array}$

4.1 Introduction 78

4.2 Direct energy transfer power system 78

4.3 EASY5 model generation and analysis 80

CHAPTER 5 ANALYSIS OF SOLAR ARRAY POWER SYSTEM MODES OF $\begin{array}{ll}\text { OPERATION } & 85\end{array}$

5.1 Introduction 85

5.2 Large-signal behavior of solar array operating points 87

5.3 Analysis of the system operating near the solar 103

5.4 Analysis of the solar array system in the 109

5.5 Analysis of modes of operation of the direct

$\begin{array}{ll}5.6 \text { Conclusions } & 134\end{array}$ 
$\begin{array}{lll}\text { CHAPTER } 6 & \text { SUBSYSTEM INTERACTION ANALYSIS } & 136\end{array}$

6.1 Introduction $\quad 136$

6.2 Load interaction analysis 137

6.3 Source interaction analysis 146

6.4 Conclusions 156

$\begin{array}{lll}\text { CHAPTER } 7 & \text { EMPIRICAL DATA MODELING AND ANALYSIS } & 158\end{array}$

7.1 Introduction 158

7.2 Model development from frequency response data 159

7.3 Review of existing complex curve fitting algorithms 161

7.4 Empirical data modeling and analysis of power systems 165

7.5 Conclusions

$\begin{array}{lll}\text { CHAPTER } 8 & \text { CONCLUSIONS } & 174\end{array}$

$\begin{array}{lr}\text { REFERENCES } & 179\end{array}$ 


\section{Chapter 1}

\section{INTRODUCTION}

The ever increasing demand on spacecraft power systems for improved efficiency and reliability, smaller size and lighter weight, coupled with continuous growth in dimension and complexity of the spacecraft's payloads, has focused attention on a major deficiency - the ability to design, test, and trouble-shoot large scale power systems.

During the past several decades, numerous efforts have been made to develop new and more powerful techniques for modeling and analysis of spacecraft power components (equipments) and subsystems. However, when the components and subsystems are interconnected to form a complex system, it is quite difficult to predict the total system response even though the behavior of individual components may be well understood and documented. This is due to many undesired interactions that exist among highly nonlinear components. The need of a comprehensive power system modeling tool is, therefore, most critical since the elaborate design verification through integrated systems hardware testing is prohibitively expensive or often impossible. This is particularly true in the future space station system since, due to the large dimension and complexity, the complete system can only be assembled in space. Therefore, a comprehensive computer model that can actually predict a system's local and global behaviors is most critical for the success of future missions.

Considering the size and complexity of todays spacecraft power 
processing systems, the digital computer is perhaps the only viable tool for system modeling and simulation. Two general classes of power system modeling programs appear in the literatures. These are :

- Generalized circuit and system analysis models.

- Dedicated models of specific systems.

The generalized circuit analysis models, as a class, are represented by programs such as SPICE, SCEPTRE, SYSCAP, ICAP, ASTAP and a number of others. All have similarities in that they offer a set of typical device modules which are assembled into a network of interconnecting nodes. This class of computer programs shares a common drawback, such that with an increasing number of components, the memory requirements increase dramatically. By use of dynamic memory management it may be possible to analyze a large-scale system, but only at the cost of a geometric increase in computation time. The labor involved in model-building is rather extensive and the resulting model, in general, lacks flexibility for future modifications. Furthermore, the analysis capability of these canned programs is limited and usually not effective for design aid or trouble-shooting.

Dedicated models of specific power systems have been used successfully but only on a limited basis. Typically this class of model is capable only of DC analysis. Execution speed and cost of such programs tend to be much less than that for generalized circuit and system routines [1]. 
Therefore, a comprehensive power system model must be capable of performing various analyses - DC, small signal and large signal transient. The model should have the following attributes :

- flexible for future modification of component models and system configuration,

- accurate to provide sufficient details about the behavior of individual components for design and troubleshooting,

- verifiable whenever equipment or subsystem experimental testing is possible,

- efficient to minimize computer core memory and computation time.

One way to develop a model with such attributes is to modularize various components of the power system. In Chapter 2, a methodology for modeling and analysis of a large scale power system is presented. A two-port subsystem coupling method is utilized and its interconnection law is defined. A system analysis technique is described that defines the total system as being a doubly terminated two-port network for an arbitrary subsystem. The system performance parameters are derived explicitly as functions of the two-port $g$-parameters of the subsystem (or component) and impedances of its terminating subsystems. 
In Chapter 3, modeling of various components of the spacecraft power system is presented. Each component is modeled as an unterminated twoport. For each nonlinear component, the time-domain simulation model and the linearized small signal model are developed. The transfer function models of the $g$-parameter of individual component and the small signal performance parameters of the terminated component are derived. 'Using the component models developed in Chapter 3, a sample system model - the direct energy transfer (DET) spacecraft power system is developed. The system model building with the host software system and its analysis capabilities are described in Chapter 4.

Using the DET system model, various types of system level analyses are performed in Chapters 5 through 7. In Chapter 5, various modes of operation of the DET system are described. The large signal behavior of the nonlinear solar array power system is analyzed using the state-plane method. The system behavior near the solar array maximum power point is discussed.

The operation of a battery discharger circuit and the design considerations suitable for a wide range of operations (i.e., from the no-load to full load) of a feedback compensator are described. The complete system mode of operation including the transition mode between the shunt mode and the battery discharging mode is illustrated.

In Chapter 6, subsystem interaction problems are illustrated. The local and global interactions are described and a methodology for the design and trouble-shooting of a system dealing with the interaction problems is presented. One of the most attractive approaches for modeling 
and analysis of complex large scale systems is the empirical data modeling technique. In chapter 7 , empirical data modeling employing a complex curve fitting technique is presented. The system level analysis using this technique is performed by defining an arbitrary subsystem as a black box for which the curve fitted analytical model is realized from the empirical data. The complex curve fitting technique is extended to derive a reduced order model for a high order subsystem. Conclusions are presented in Chapter 8 . 


\section{Chapter 2}

\section{METHODOLOGY OF MODELING AND ANALYSIS OF SPACECRAFT POWER SYSTEMS}

\subsection{Introduction}

This chapter discusses a modeling and analysis methodology of large scale systems. Section 2.2 uses the modular concept that a large scale system may be defined as an interconnection of many individual subsystems. This idea is particularly attractive for spacecraft power systems consisting of many individual components since each component may be supplied by a different vendor where performance parameters and design information are documented as a stand alone equipment (component). By defining standardized interconnection laws between each component, a system can be analyzed and synthesized using the information of the individual component modules. In Section 2.3 the terminal characteristics of a singly and doubly terminated two-port subsystem are derived as transfer function models for a system linear analysis. In Section 2.4 , based on the model representation derived in Section 2.3 , system stability is analyzed. This section further addresses the compatibility and incomparability of individual components in terms of their terminal characteristics and the interconnection schemes. Performance degradations and system instability as a result of undesired component interactions are illustrated. Conclusions are presented in Section 2.5. 


\subsection{Modeling approach of large scale systems}

As discussed in Chapter 1, defining a complete spacecraft power system entirely in terms of circuit elements is not feasible. A practical approach is to consider that a large scale system can be defined by the interconnection of many smaller subsystems. The system dynamics, therefore, stem from the individual subsystems and their interconnection laws that identify the signal flows among them. Due to the complexity of the individual components and their interconnection scheme it is usually very difficult to predict the system's overall behavior. A desirable analysis or synthesis procedure for a large scale system is the separation of these two levels (component and system). One thus prefers an analysis or synthesis procedure to be based, on one hand, on "local" subsystems and, on the other hand, on the "global" interconnection laws.

In order to utilize the modular concept, each component should be modeled as an unterminated two-port network because the terminal characteristics of both source and load are unknown until the complete system is configured. The interconnection law should not only describe accurately the signal flows between the subsystems but also be standardized. There exist several kinds of two-port representations such as impedance, admittance, chain, and hybrid matrices. The impedance or admittance matrices cannot be used as a standard interconnection law because their inputs and outputs are in alternate forms. The chain matrix is not as flexible as the hybrid matrix because it only characterizes a system in uni-direction. For example, the chain matrix can describe port-1 of a two-port subsystem as a function of its load (which may be connected in 
cascade in $n$ two-port subsystems) that is connected at port-2 as shown in Eq. (2.1).

$$
\left[\begin{array}{c}
e_{1}^{1} \\
f_{1}^{1}
\end{array}\right]=\left[c_{1}\right]\left[\begin{array}{l}
c_{2} \\
\end{array}\right]\left[\begin{array}{l}
c_{n} \\
f_{2} n
\end{array}\right]
$$

where the subscripts and superscripts represent the port numbers and the $j^{\text {th }}$ component, respectively.

However, quite often, it is necessary to analyze a two-port subsystem as functions of both its source and load. Thus, the information flow can be obtained in both directions. Fig.2.1 illustrates the interconnection law for unterminated modules. Each component, in general, receives two inputs $v_{1}$ and $i_{2}$ from the preceding and following modules, and provides two outputs $i_{1}$ and $v_{2}$ to the adjacent modules. The terminal characteristics of each component are described by the matrix equation called "hybrid g-parameters" in Eq. (2.2).

$$
\left[\begin{array}{l}
i_{1} \\
v_{2}
\end{array}\right]=\left[\begin{array}{ll}
g_{11} & g_{12} \\
g_{21} & g_{22}
\end{array}\right] \cdot\left[\begin{array}{l}
v_{1} \\
i_{2}
\end{array}\right]
$$

For modeling a two-port, one can always define the output and input variables as shown in Fig.2.1. For example, a single stage LC low-pass filter can be modeled with state equations as shown in Fig.2.2. Since these unterminated two-port components have the same interconnection law, 

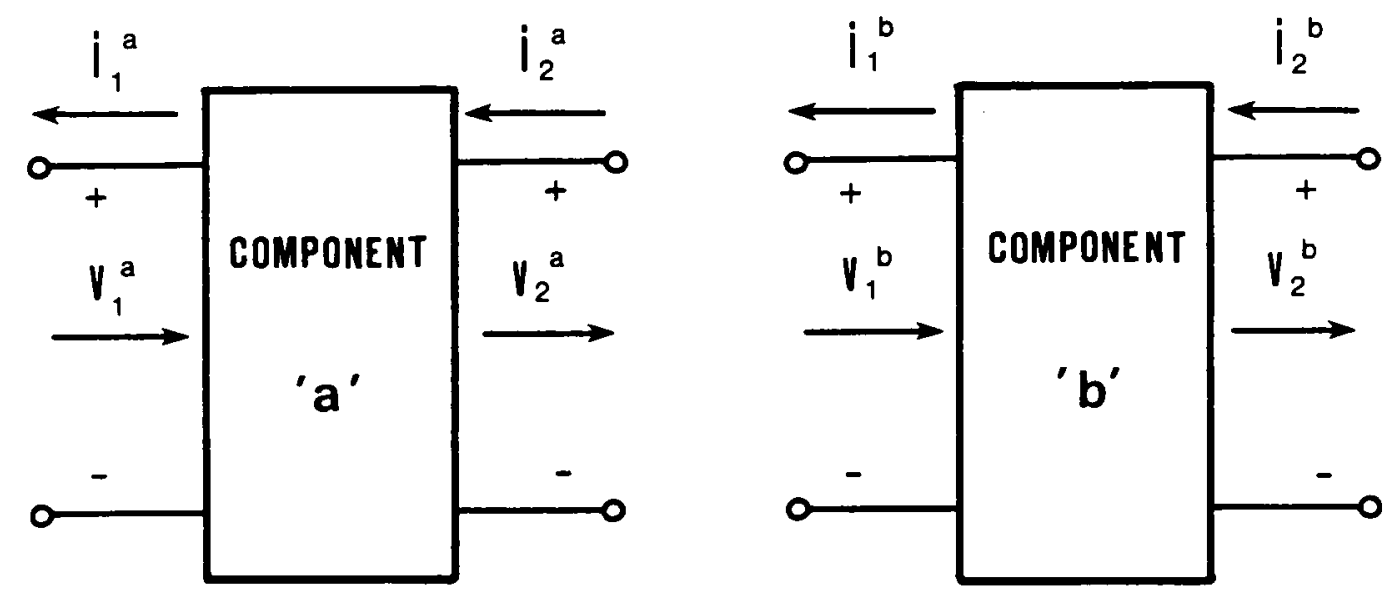

Figure 2.1 Two-port subsystem coupling using the hybrid G-matrix 


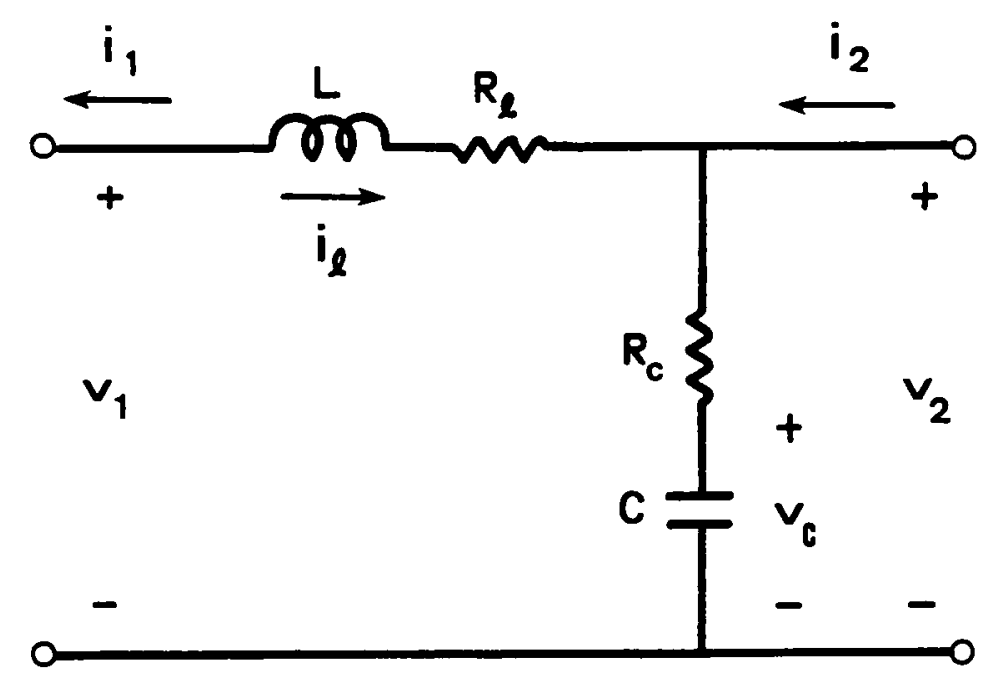

$\left[\begin{array}{c}i_{\ell} \\ v_{c}\end{array}\right]=\left[\begin{array}{cc}\frac{-\left(R_{\ell}+R_{c}\right)}{L} & \frac{-1}{L} \\ \frac{1}{C} & 0\end{array}\right]\left[\begin{array}{c}i_{\ell} \\ v_{c}\end{array}\right]+\left[\begin{array}{cc}\frac{1}{L} & \frac{-R_{c}}{L} \\ 0 & \frac{1}{C}\end{array}\right]\left[\begin{array}{c}v_{1} \\ i_{2}\end{array}\right]$

$\left[\begin{array}{l}i_{1} \\ v_{2}\end{array}\right]=\left[\begin{array}{ll}-1 & 0 \\ R_{c} & 0\end{array}\right]\left[\begin{array}{l}i_{\ell} \\ v_{c}\end{array}\right]+\left[\begin{array}{ll}0 & 0 \\ 0 & R_{c}\end{array}\right]\left[\begin{array}{l}v_{1} \\ i_{2}\end{array}\right]$

Figure 2.2 Example of an unterminated two-port modeling with state equations 
they can be interconnected arbitrarily to form a system.

Having identified the two-port component coupling approach, efforts have been made to find a software system which can be adapted to multiport component modeling and system integration. The program EASY5 developed by Boeing Computer Service has been selected as the host software system.

The EASY5 software [2] provides a modular approach to dynamic system model building and analysis. It was chosen particularly for its effective means of assembling complex component modules into a system. Predefined component modules can be stored in the EASY5 component library. The component modules can have several input and output ports and, in each port, information can flow into and out of the port. All detailed connections of signal paths between component blocks are programmed with minimal user intervention. More details about the EASY5 program will be described in Section 4.2 .

For a large scale spacecraft power system, the large signal performance evaluation is invariably limited to tactics closely identified with time-domain simulation techniques. The actual operating point trajectories or transient responses due to the large signal disturbances can be simulated by a digital computer. In this way, the existence of unstable solutions can be observed along with peak values and actual waveforms. Thus, the model development of each nonlinear component of a spacecraft power systems involves two separate models, the large signal time-domain simulation model and the small signal linearized model. 
For time-domain simulation, the EASY5 numerical integration routine calculates current values of the states and output variables for each component from the input values. It then passes along the output values according to the interconnection law. There are seven different types of numerical integration routines available in EASY5. Each offers different numerical stability and computational efficiency dependent on the type of nonlinearity of a system. To examine the approximate large signal behavior of a system analytically, rather than by time-domain simulation, one may simplify the system focusing on a particular component of interest. For instance, the large signal behavior of the solar array system's operating point can be analyzed by simplifying the system to a second order nonlinear system as illustrated in Chapter 5.

For the small signal linear analysis, various types of classical linear system theories can be adapted. The methodology of large scale system analysis is described in the following sections.

\subsection{Two-port transfer function models}

To analyze the two-port component or subsystem in Fig.2.1, one needs to identify the G-matrix in Eq.(2.2). From Eq.(2.2), the g-parameters can be described in the form of transfer functions. As Fig.2.3 illustrates, each parameter can be identified by using an appropriate sinusoidal current or voltage excitation. It is important to notice that the g-parameters are identified in terms of measurable (observable) quantities, thus the model can be verified. This modeling technique is particularly useful when the component is best treated as a black box due 

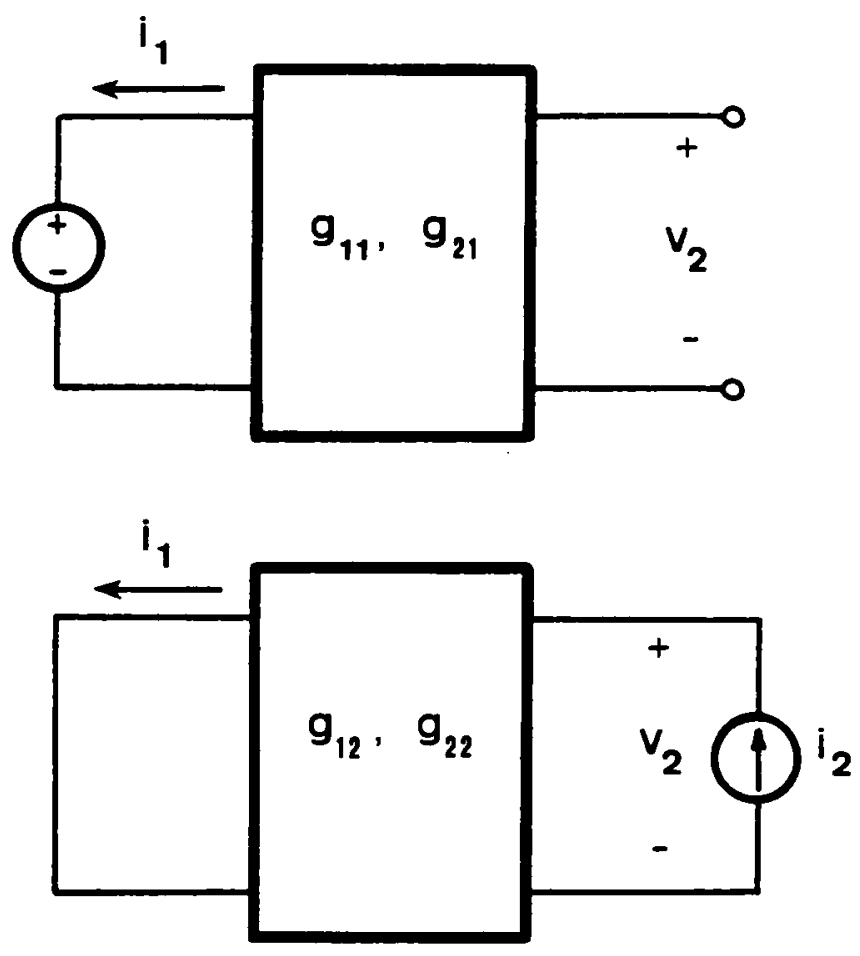

$$
\begin{aligned}
& g_{11}=\left.\frac{i_{1}}{v_{1}}\right|_{i_{2}=0} ; \text { open circuit input admittance } \\
& g_{21}=\left.\frac{v_{2}}{v_{1}}\right|_{i_{2}=0} ; \text { open circuit voltage trnasfer function } \\
& g_{12}=\left.\frac{i_{1}}{i_{2}}\right|_{1}=0 \quad ; \text { short circuit current transfer function } \\
& g_{22}=\frac{v_{2}}{i_{2}} \mid \begin{array}{ll}
v_{1}=0 & ; \text { short circuit output impedance }
\end{array}
\end{aligned}
$$


to its complexity or unknown nature. The empirical data of the two-port g-parameters can easily be obtained, from which an analytical closed-form model is realized employing the complex curve fitting technique. The empirical data modeling approach will be discussed in Chapter 7.

The two-port modeling approach allows one to analyze the complete system with a focus on the local behavior of a particular component or subsystem. As far as the two-port subsystem is concerned, the entire system can be considered as a doubly terminated two-port system as illustrated in Fig.2.4. The quantities $Z_{S}$ and $Z_{L}$ are the source and load impedances seen by the two-port subsystem. Notice that the equivalent circuit may be obtained by replacing the generator and $Z_{S}$ by its Norton equivalent in the circuit. As shown in Fig. 2.4 four important parameters can be realized in terms of the two port $g$-parameters $Z_{S}$ and $Z_{L}$.

$$
\begin{gathered}
G_{v}=\frac{g_{21} \cdot z_{L}}{Z_{L}-z_{S} \cdot \Delta G+g_{11} \cdot z_{S} \cdot Z_{L}} \\
G_{i}=\frac{g_{11} \cdot g_{22}+g_{12} \cdot g_{21}-Z_{L}}{g_{21}} \\
Y_{i}=\frac{g_{11}+\Delta G / Z_{L}}{1+g_{22} / Z_{L}} \\
Z_{0}=\frac{g_{22}-\Delta G Z_{S}}{1-g_{11} \cdot Z_{S}}
\end{gathered}
$$




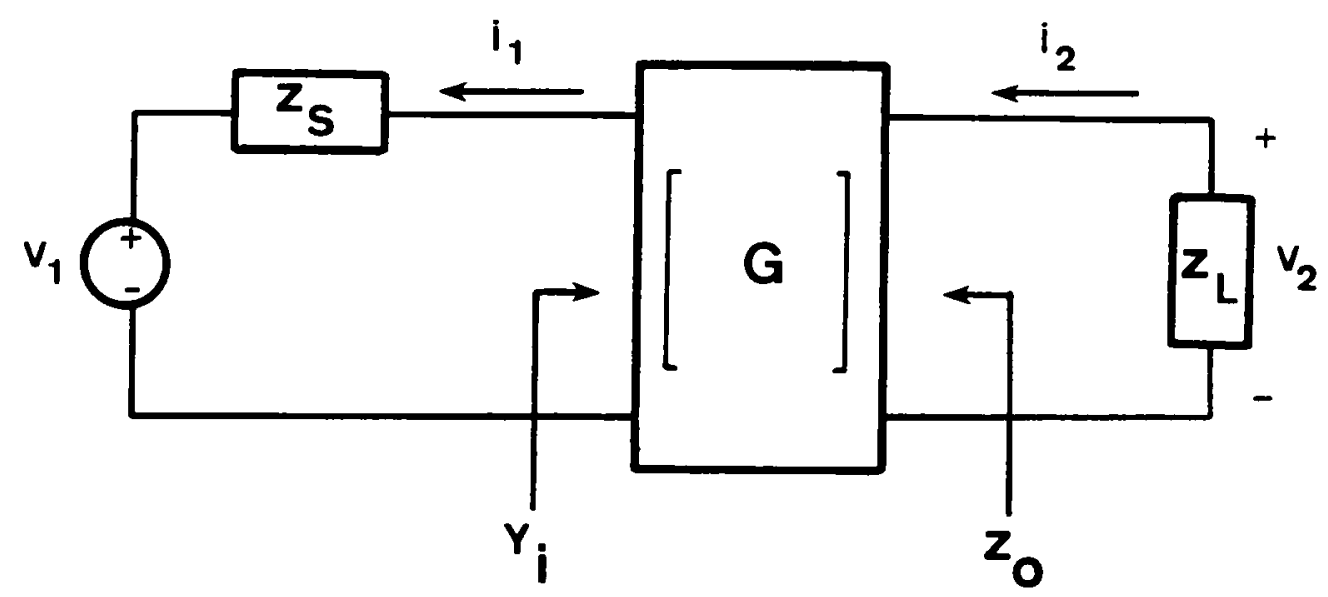

$$
\begin{array}{ll}
G_{v}=\frac{v_{2}}{v_{1}} & ; \text { forward voltage transfer function } \\
G_{i}=\frac{i_{1}}{i_{2}} & ; \text { reverse current transfer function } \\
Y_{i}=\frac{i_{1}}{v_{1}} & ; \text { load terminated input admittance } \\
Z_{0}=\frac{v_{2}}{i_{2}} & ; \text { source terminated output impedance }
\end{array}
$$

Figure 2.4 Transfer functions of the doubly terminated two-port 
where

$$
\Delta G=g_{11} g_{22}-g_{12} g_{21}
$$

These parameters play important roles in the analysis of the system dynamics especially the interactions among the various subsystems.

\subsection{System analysis approach using the transfer function models}

One of the major concerns in analyzing the large scale spacecraft power system deals with undesired interactions among components and subsystems. As a system becomes more complex, many undesired interactions occur and they create a large degree of uncertainty in the system response and stability even though each component may be well-behaved and understood. Using the presently existing modeling techniques it may be possible to simulate the entire system by a digital computer. However, the results obtained from the computer simulation often do not provide physical insight and thus give very little design information for future design changes and trouble-shootings. Based on the modeling approach described in Section 2.3 the system can be analyzed with an emphasis on either the local behavior or the global behavior. The analysis allows one to trouble-shoot the interaction problem at any system level.

From Eqs. (2.3) and (2.4) the stability of the total system can be directly analyzed by investigating the poles of the transfer function $G_{v}$ and $G_{i}$. However, since $G_{v}$ and $G_{i}$ may be of complex form, interactions among $Z_{S}, Z_{L}$, and the two-port subsystem may not be easily obtainable. 
Instead, one can investigate the source and load interaction problems using the quantities $Y_{i}$ and $Z_{0}$ in Eqs.(2.5) and (2.6), as illustrated in Fig. 2.5.

The line transfer functions in Fig.2.5 can be written as follows. From Fig.2.5a,

$$
\mathrm{H}_{\mathrm{L}}=\frac{\mathrm{v}_{2}}{\mathrm{v}_{\mathrm{s}}}=\frac{\mathrm{Z}_{\mathrm{L}}}{\mathrm{Z}_{\mathrm{O}}+\mathrm{Z}_{\mathrm{L}}}=\frac{1}{1+\mathrm{Z}_{\mathrm{o}} / \mathrm{Z}_{\mathrm{L}}} \equiv \frac{1}{1+\mathrm{T}_{\mathrm{L}}}
$$

and from Fig.2.5b,

$$
H_{S}=\frac{v_{1}}{v_{s}}=\frac{1 / Y_{i}}{1 / Y_{i}+Z_{S}}=\frac{1}{1+Z_{S} \cdot Y_{i}} \equiv \frac{1}{1+T_{S}}
$$

Eqs.(2.7) and (2.8) shows that the system characteristic equation is $1+$ $\mathrm{T}_{\mathrm{S}}=0$ or $1+\mathrm{T}_{\mathrm{L}}=0$, respectively. Hence, the system is stable if the quantity $\mathrm{T}_{\mathrm{S}}$ in Eq.(2.7) or $\mathrm{T}_{\mathrm{L}}$ in Eq. (2.8) satisfies the Nyquist criterion that is, the system is stable if the number of counterclockwise encirclements of the point $(-1,0)$ in $s-p l a n e$ of the polar plot of $T^{\prime} s$ is equal to the number of the right-half plane (RHP) poles in $T$. This statement still may be too general unless the number of RHP poles in $T_{S}$ or $\mathrm{T}_{\mathrm{L}}$ are identified. If we assume that the elements in Eqs.(2.7) and (2.8) are all stable quantities, i.e. no RHP poles, then the Nyquist 


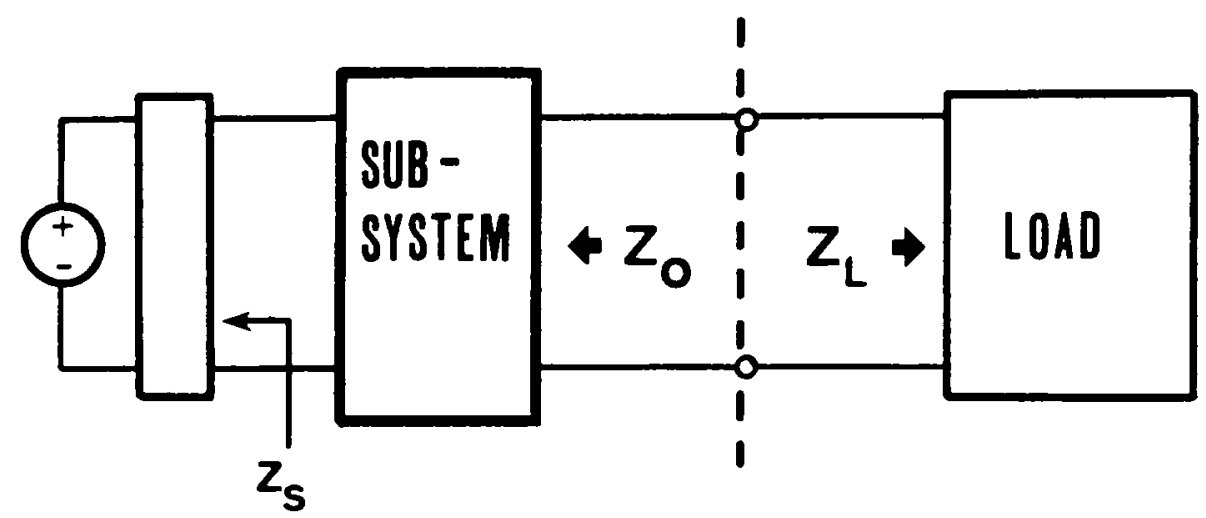

a.

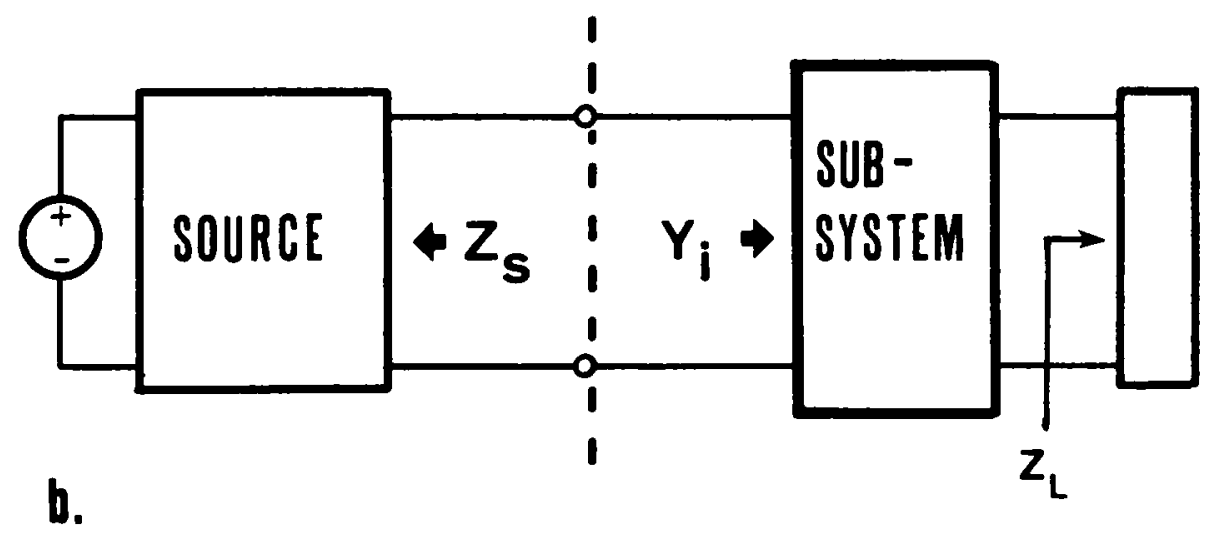

Figure 2.5 Subsystem interaction parameters

(a) load interaction ( $Z_{0}$ vs $Z_{L}$ )

(b) source interaction $\left(Z_{s} \vee s \quad Z_{j}\right)$ 
criterion can be restated as; the system is stable if the polar plot of $\mathrm{T}_{\mathrm{S}}$ or $\mathrm{T}_{\mathrm{L}}$ does not encircle the point $(-1,0)$.

To analyze the interaction problems of a two-port with $Z_{S}$ and $Z_{L}$, it is necessary to check the above assumption that the quantities $Z_{S}, Z_{L}$, $Y_{i}$ and $Z_{0}$ are all stable (i.e., no RHP poles). If any of these are unstable then an interaction has already occurred with an unstable quantity and the analysis should trace back until the point of the undesired interaction is found. To do this consider Fig.2.6 which elaborates the system in Fig.2.4. The quantities in Eqs.(2.3) through (2.6) of the $j^{\text {th }}$ component can be expressed in terms of the preceding $\left(j-1^{\text {th }}\right)$ and the following $\left(j+1^{\text {th }}\right)$ components.

$$
\begin{aligned}
& Z_{S}^{j}=z_{o}^{j-1} \\
& Z_{L}^{j}=1 / Y_{i}^{j+1}
\end{aligned}
$$

From Eqs. (2.5) and (2.6),

$$
\begin{aligned}
& Z_{0}^{j}=\frac{g_{22}^{j}-\Delta G^{j} \cdot z_{S}^{j}}{1-g_{11}^{j} \cdot z_{S}^{j}}=\frac{g_{22}^{j}-\Delta G \cdot z_{o}^{j-1}}{1-g_{11}^{j} \cdot Z_{o}^{j-1}} \\
& Y_{i}^{j}=\frac{g_{11}^{j}+\Delta G^{j} / Z_{L}^{j}}{1+g_{22}{ }^{j} \cdot z_{L}^{j}}=\frac{g_{11}^{j}+\Delta G \cdot Y_{i}^{j+1}}{1+g_{22}^{j} \cdot Y_{i}^{j+1}}
\end{aligned}
$$

From Eqs. (2.9) through (2.12) the stabilities of $Z_{S}, Z_{L}, Y_{i}$ and $Z_{0}$ for 


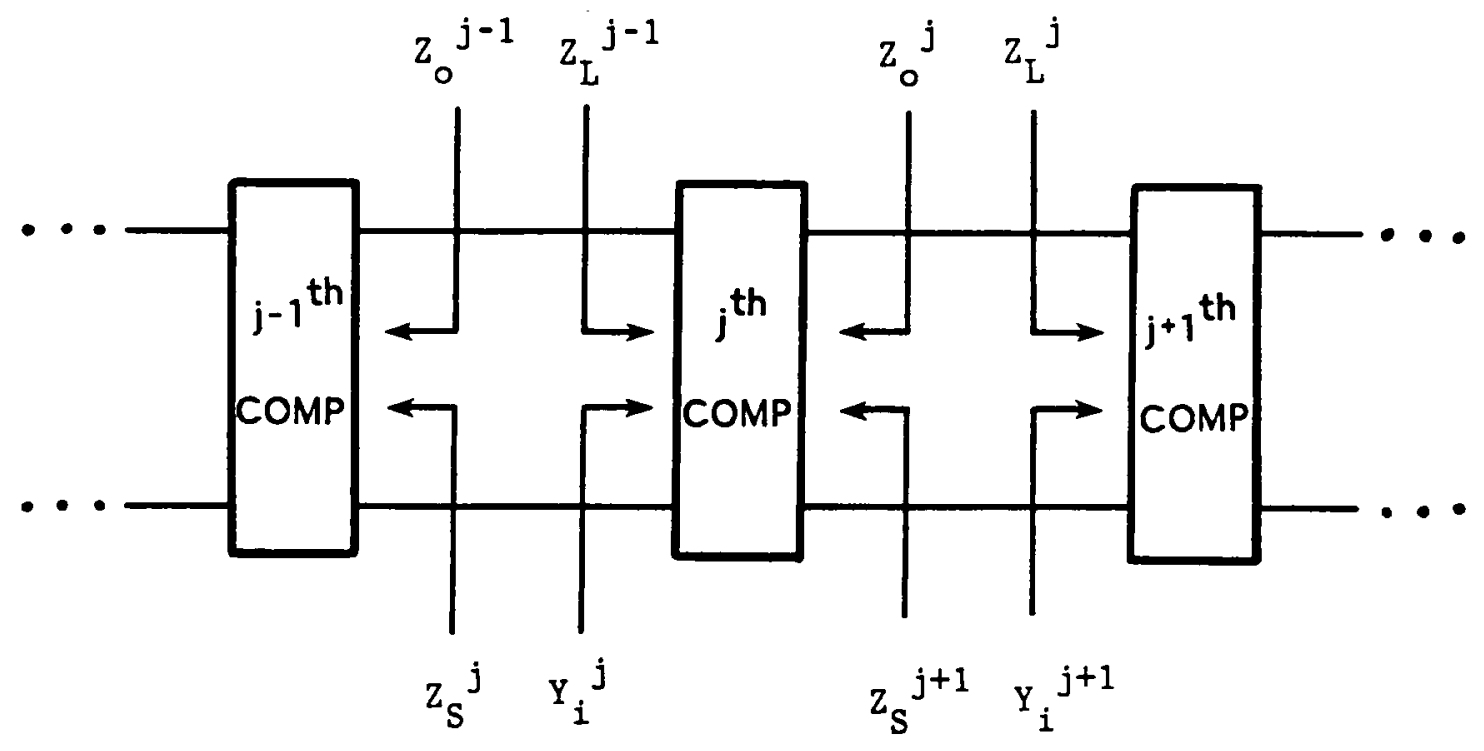

Figure 2.6 Realization of impedances for cascade components 
any two-port can easily be checked and the possible undesired interaction points detected. For instance, the stability of output impedance of the $j^{\text {th }}$ component $Z_{0}^{j}$ can be determined from Eq. (2.11) by applying the Nyquist criterion to the product of $-g_{11}{ }^{j}$ and $z_{0}^{j-1}$. Since each unterminated component ( $g$-parameters) is assumed to be stable and if $z_{0}^{j}$ is unstable while $Z_{0}{ }^{j-1}$ is stable, then the undesired interaction occurs between the $j^{\text {th }}$ component and its source impedance $z_{S}{ }^{j}$. The same argument holds for the load interactions such that if $Y_{i}{ }^{j}$ is unstable while $Y_{i}^{j+1}$ is stable then the undesired interaction occurs between the $j^{\text {th }}$ component and its load impedance $z_{L}^{j}$. In other words, the stability of the doubly terminated two-port component can be established as follows. First, using Eqs. (2.9) through (2.12) the stability of $Z_{S}$ and $Z_{L}$ must be examined then using Eqs. (2.11) and (2.12) the stability of $Y_{i}$ and $Z_{0}$ (singly terminated two-port) can be determined. If $Y_{i}$ and $Z_{0}$ are stable, which implies $Z_{S}$ and $z_{L}$ are also stable, then the stability of the doubly terminated twoport system can be established by applying the Nyquist criterion to $\mathrm{T}_{S}$ and $\mathrm{T}_{\mathrm{L}}$ in Eqs.(2.7) and (2.8).

\subsection{Conclusions}

In this chapter the proposed modeling and analysis techniques of large scale systems were discussed. A two-port subsystem coupling method is utilized such that a system is broken into manageable pieces. Each component is modeled as an unterminated two-port and the system is configured according to the interconnection law. 
The stability of a linearized large scale system can be analyzed with a focus on either local or global behavior. The analysis not only determines the stability of the total system but also identifies the undesired interactions which occur at various component levels. Thus, the design criteria for components and their integration can be established. The modular approach allows one to pinpoint the undesired interaction point to assist in trouble-shooting. The modular approach also allows the analysis to be performed with well-defined physical quantities (i.e., impedances) which are also observable and measurable both analytically and empirically. The analys is technique is based on well-known classical linear circuit theory, two-port network theory and Nyquist theory so that any practicing engineer can utilize the technique for design, analysis and trouble-shooting a large-scale system. Various types of the system level analyses for the Direct Energy Transfer spacecraft system are performed in Chapters 5 through 7. 


\section{Chapter 3}

\section{SPACECRAFT COMPONENTS MODELING AND ANALYSIS}

\subsection{Introduction}

Components of spacecraft power systems are inherently nonlinear. As a result, their behaviors due to large signal and small signal disturbances can be quite different. For the analysis of the small signal dynamics, such as stability, input/output impedances, and audiosusceptibility, the model can be developed by linearizing the nonlinear components about an operating point under the small signal assumptions. These small signal models are very useful since all of the well established linear system theories can be applied. Also their analytical closed forms provide useful information for designing the system. However, the small-signal models can not predict the large-signal behavior such as stability in large, initial start-up and step transients, and other nonlinear modes of operation (i.e., protection, saturation, etc.). Therefore, it is necessary to generate a small signal model and large signal model for these nonlinear components to analyze the following performance categories: dc, small signal ac, and large signal transient responses.

It is desirable that various components in the spacecraft power system be modeled with maximum flexibility, using any one of the following representations: state equations, transfer functions, empirical data, and combinations of those. One may choose one of these modeling techniques 
best suited for a particular component. For instance, quite often a component can be virtually a black box due to either complexity or unknown nature, then empirical data modeling is the only resort. For a component of piecewise linear nature, such as switching converters, the state space modeling is most convenient. For a linear component, the transfer function model often suits best because it provides more design insight. Integrating these component models in different forms can be done with minimum user's intervention employing the host software system EASY5. Details for the system modeling are discussed in Chapter 4.

As described in Chapter 2, characteristics of the g-parameters for each component should be analyzed as an unterminated two-port network. The system level analysis can then be carried out based on the g-parameters of individual components. In this chapter, modeling and analysis of the following spacecraft components are discussed.

- Switching regulator - Section 3.2

- Solar array - Section 3.3

- Shunt regulator - Section 3.4

- Filter / cable - Section 3.5

- Payload - Section 3.6 


\subsection{Switching regulator modeling}

Because of high efficiency, small size and light weight, switching regulators are widely used in spacecraft power systems. Switching regulators can be characterized, as shown in Fig.3.1, by the three basic functional blocks: power stage, analog feedback controller, and digital signal processor.

The power stage consists of energy storage elements and switches. Transfer of the input power to the load is controlled by the duty ratio of the switch (transistor). The analog feedback controller usually contains an error amplifier and a compensation network. For a single loop control it senses the output (load voltage), and for a multiple loop control it senses the output and states (i.e., load voltage, inductor current, capacitor voltage). The digital signal processor includes a ramp generator, a comparator, and latches. It takes the control voltage from the analog feedback and converts to the pulse width modulated (PWM) signal which controls the switches in the power stage switch.

Switching regulators are inherently nonlinear and discrete. The power stage has several different topological modes controlled by the switch(es) whose switching time is PWM controlled by the feedback controller. The state equations, in general, cannot be solved unless the system is linearized about an operating point. The linearized model can be used to analyze the small signal behavior about an operating point using the relatively simple linear system analys is techniques such as Bode plots, root locus, etc. 


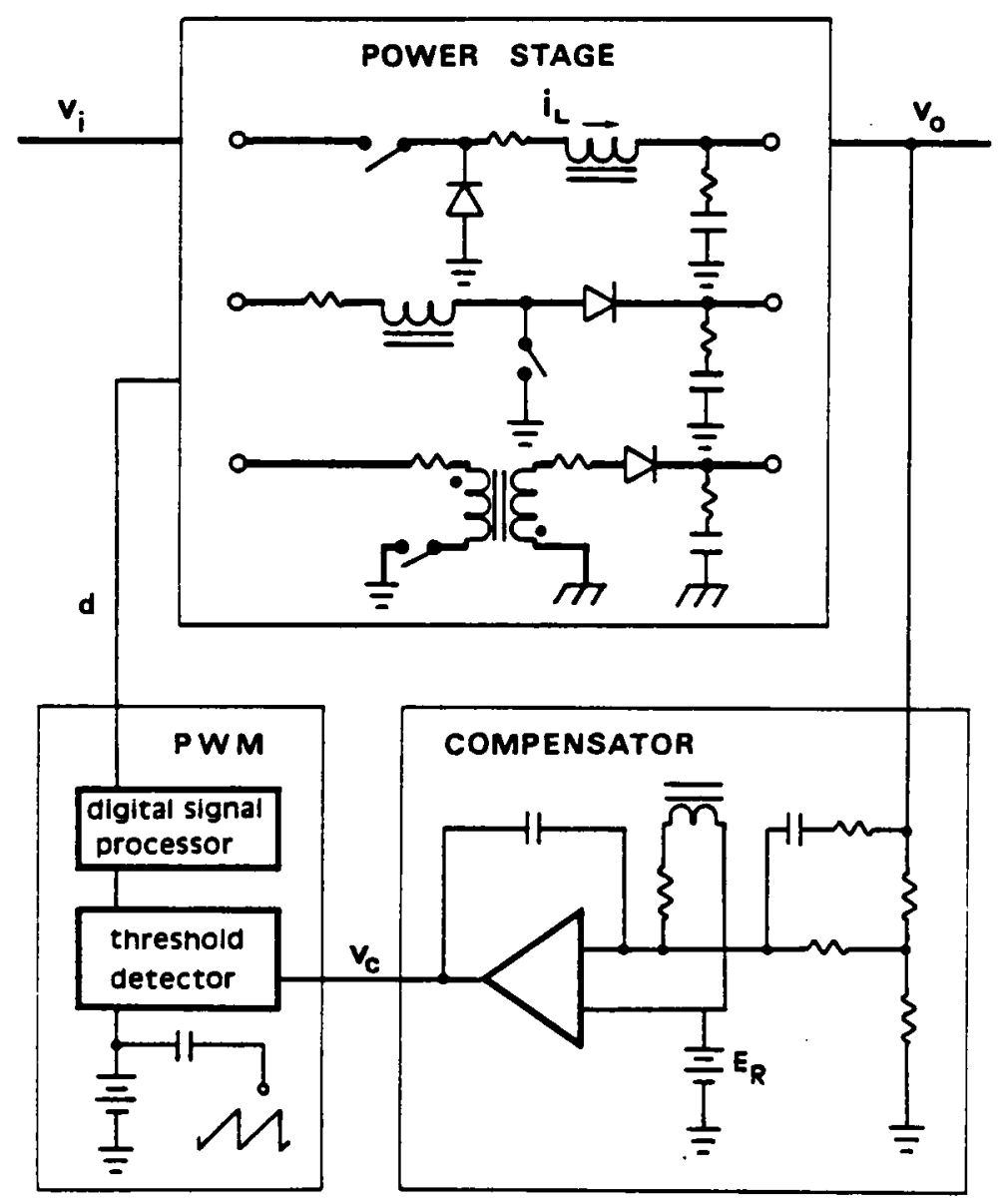

Figure 3.1 Switching regulator model 
Since there exist many different types of power stage topologies and control methods, the large signal behaviors of a regulator depend largely on the type of power circuit topology and control. Thus, for maximum flexibility, it is best to develop models for each functional block as independent modules. A regulator can then be configured by collecting appropriate pre-defined modules for each functional block. Also any of these block modules can be freely replaced with alternatives, it provides maximum flexibility in building a variety of regulators. Development of each functional block are as follows.

\subsubsection{Power stage modeling}

Generally, three different circuit topologies exist in the course of a complete switching cycle. A converter is characterized by three sets of state equations during a switching cycle as follows

$$
\begin{aligned}
& \dot{x}=A_{1} x+B_{1} u \\
& y=C_{1} x+E_{1} u, \text { for interval } d_{n 1} T_{s} \\
& \dot{x}=A_{2} x+B_{2} u, \\
& y=C_{2} x+E_{2} u, \text { for interval } d_{n 2} T s \\
& \dot{x}=A_{3} x+B_{3} u, \\
& y=C_{3} x+E_{3} u, \text { for interval } d_{n 3} T s
\end{aligned}
$$


Here $x$ is the state vector, $u$ the input vector and $T_{s}$ the switching period. $A_{i}$ and $B_{i}(i=1,2,3)$ are square matrices which describe the three circuit topologies and the effects of the input vector $u$. The switch duty ratio is represented by the fractional quantity $d_{n 1}$, and $d_{n 1}+d_{n 2}+d_{n 3}$ $=1$. These three matrix equation can be combined into one by using the three switching functions shown in Fig.3.2. With use of these switching functions, a single state equation suffices to describe the converter as in $\mathrm{Eq} \cdot(3.4)$.

$$
\begin{aligned}
& \dot{x}=\sum_{i=1}^{3}\left[A_{i} x+B_{i} u\right] d_{i}(t), \\
& y=\sum_{i=1}^{3}\left[C_{i} x+E_{i} u\right] d_{i}(t), \quad i=1,2,3
\end{aligned}
$$

where $d_{i}$ 's are either 0 or 1 depending on the specific time interval involved.

Eq. (3.4) is not only discrete but also nonlinear because $d_{i}(t)$ is a function of the state vector $x$ and the input vector $u$. The power stage model showing in Eq.(3.4) is directly implemented with the appropriate switching function for the time-domain model. The intervals defined above include the possible inductor current discontinuous conduction mode (DCM). For the continuous conduction mode (CCM), there are only two switching intervals, $d_{n 1} T_{s}$ and $d_{n 2} T_{s}$. In the time-domain model, these two different modes of operation (DCM or CCM) are naturally determined depending on circuit parameter values and input and output requirements. 


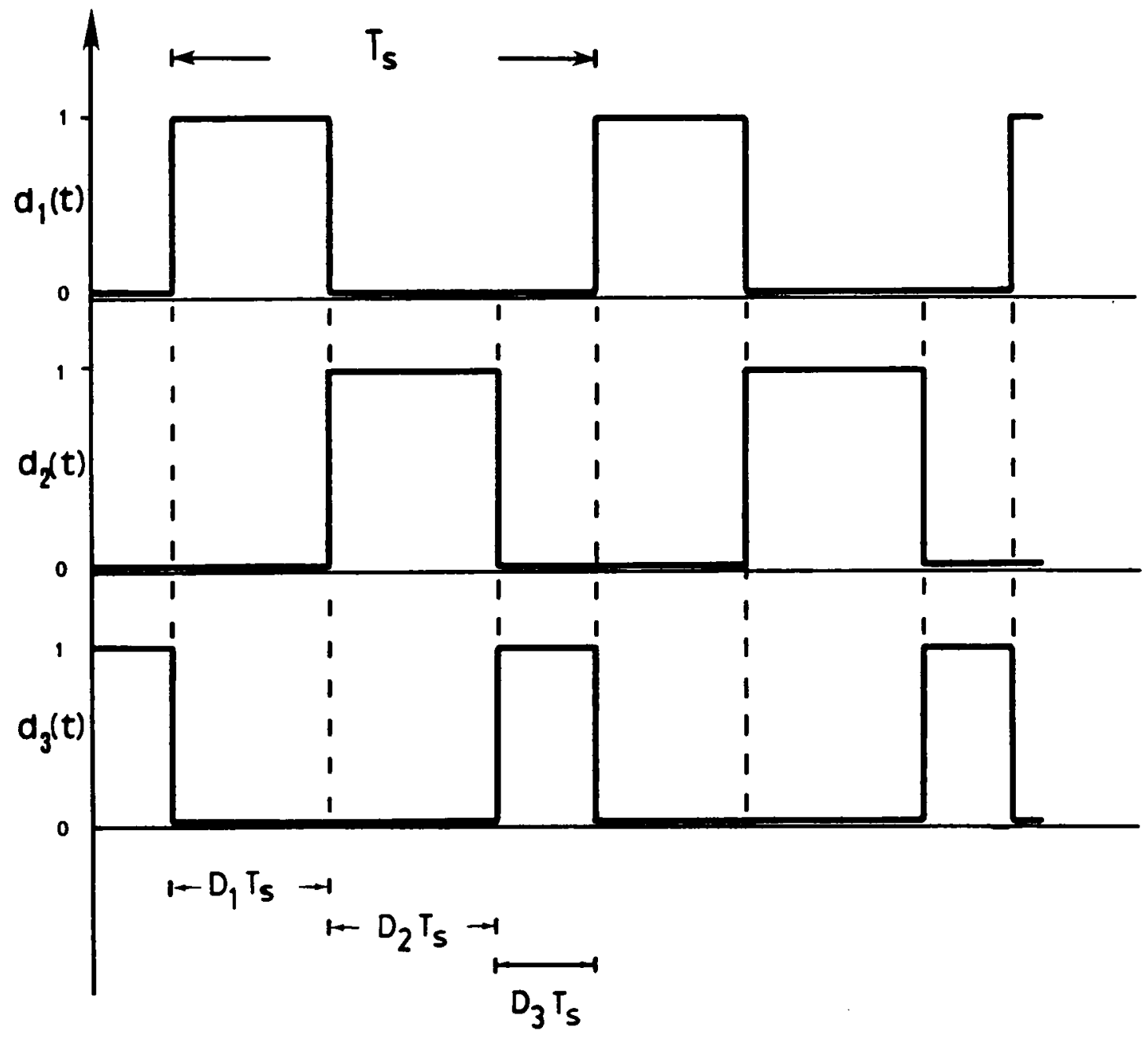

Figure 3.2 Switching functions 
However, the small signal models for CCM and DCM are quite different because of the additional constraints of the state,

$$
\frac{d i}{d t}=0
$$

and

$$
i=f\left(u, y, d_{n 1}, L, T_{s}\right)
$$

Therefore, it is necessary to include a step to determine whether the converter is operated in CCM or DCM for the small-signal model by checking the boundary condition between these two modes. From Eq. (3.4), the small signal power stage model can be generated as follows.

\section{Linear small signal modeling of switching converter power stage}

For the small signal model, there exist various types of techniques such as state space averaging [3], discrete-averaging [4] and discrete technique [5]. Among these, the state space averaging technique is chosen for this study because it is simple and provides physical insight into the system, thus it is better suited especially for large system analys is. The small signal modeling in this section will be carried out for the case of the constant frequency controlled regulator operating in the CCM. The fundamentals of the state-space averaging are reviewed as follows.

Averaging: First the switching functions $d_{i}(t)$ are averaged as shown in Fig.3.3. For the CCM where $d_{n 3}=0$, let the averaged values of the 
switching functions be

$$
\begin{aligned}
& \bar{d}_{1}(t)=d \\
& \bar{d}_{2}(t)=1-\bar{d}_{1}(t)=d^{\prime}
\end{aligned}
$$

Both dynamic and static equations for the two (in CCM) switched intervals in Eq. (3.4) are averaged by summing the equations for each interval $d_{n i} T_{s}$ multiplied by the averaged switching function $d_{i}(t)$. It results in the following continuous system which represents the average effect of the circuit across the whole period $T_{s}$.

$$
\begin{aligned}
& \dot{x}=\left(A_{1} d+A_{2} d^{\prime}\right) x+\left(B_{1} d+B_{2} d^{\prime}\right) u \\
& y=\left(C_{1} d+C_{2} d^{\prime}\right) x+\left(E_{1} d+E_{2} d^{\prime}\right) u
\end{aligned}
$$

where $\mathbf{x}$ and $\mathrm{y}$ represent the average state and output.

Perturbation : Suppose that the duty ratio changes from cycle to cycle, that is, $d=D+\hat{d}$ where $D$ is the steady state (dc) duty ratio and $\hat{\mathrm{d}}$ is a superimposed (ac) variation, as shown in Fig.3.3. The notation used throughout this section is that dc or steady state values are represented by upper case letters, and perturbations by lower-case with carets. The corresponding perturbations of other quantities can also be expressed as 


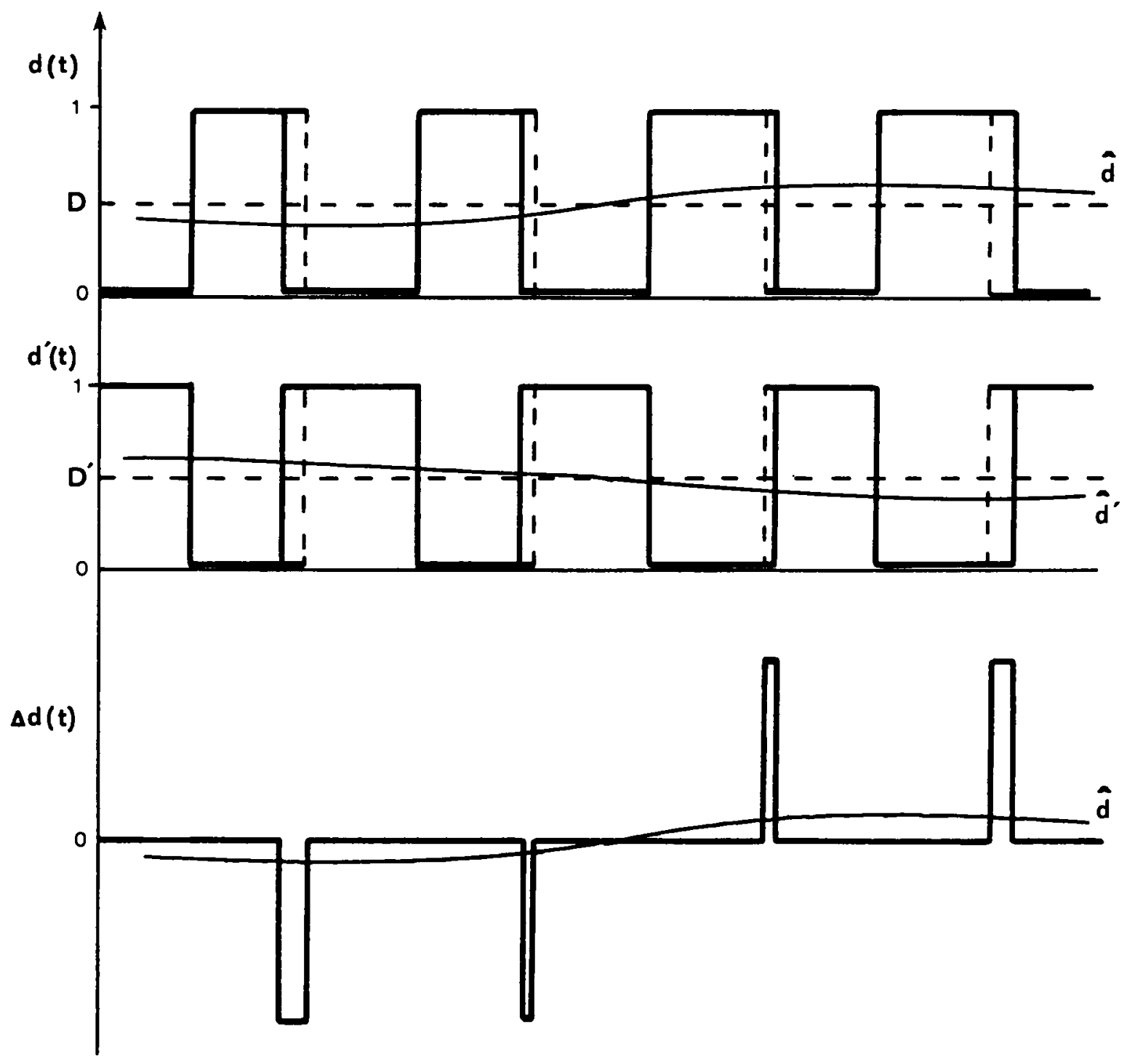

Figure 3.3 Averaging the switching functions

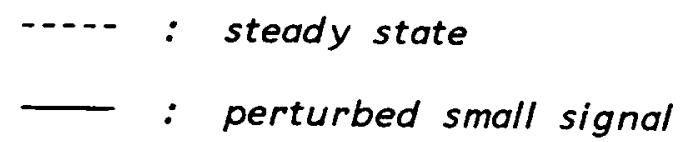




$$
\begin{aligned}
& \mathrm{d}^{\prime}=1-\mathrm{d}=(1-\mathrm{D})-\hat{\mathrm{d}}=\mathrm{D}^{\prime}-\hat{\mathrm{d}} \\
& \mathrm{x}=\mathrm{x}+\hat{\mathrm{x}}, \mathrm{u}=\mathrm{U}+\hat{\mathrm{u}}, \mathrm{y}=\mathrm{Y}+\hat{\mathrm{y}},
\end{aligned}
$$

Substituting these into the averaged equation ( 3.8$)$, it becomes

$$
\begin{aligned}
\dot{\hat{x}}=A X+B U+A \hat{x}+B \hat{u} & +\left[\left(A_{1}-A_{2}\right) \hat{x}+\left(B_{1}-B_{2}\right) U\right] \hat{d} \\
+ & {\left[\left(A_{1}-A_{2}\right) \hat{x}+\left(B_{1}-B_{2}\right) \hat{u}\right] \hat{d} } \\
\hat{y}=C X+E U+C \hat{x}+E \hat{u}+ & {\left[\left(C_{1}-C_{2}\right) x+\left(E_{1}-E_{2}\right) U\right] \hat{d} } \\
+ & {\left[\left(C_{1}-C_{2}\right) \hat{x}+\left(E_{1}-E_{2}\right) \hat{u}\right] \hat{d} }
\end{aligned}
$$

where

$$
\begin{aligned}
& A=D A_{1}+D^{\prime} A_{2}, \quad B=D B_{1}+D^{\prime} B_{2} \\
& C=D C_{1}+D^{\prime} C_{2}, \quad E=D E_{1}+D^{\prime} E_{2}
\end{aligned}
$$

The perturbed state-space description is nonlinear owing to the presence of the product of the two time dependent quantities $\hat{x}$ and $\hat{d}$.

Linearization: Let us now make the smal1 signal assumption such that

$$
\frac{\hat{\mathrm{u}}}{U} \ll 1, \frac{\hat{\mathrm{d}}}{\mathrm{D}} \ll 1, \frac{\hat{\mathrm{x}}}{\mathrm{X}} \ll 1 \text {, and } \frac{\hat{\mathrm{y}}}{\mathrm{Y}} \ll 1
$$


Using these small signal approximations, the nonlinear second order terms in Eq.(3.10) can be neglected, and the following linear time invariant system is obtained:

$$
\begin{aligned}
& \hat{x}=A \hat{x}+B \hat{u}+P \hat{d} \\
& \hat{y}=C \hat{x}+E \hat{u}+Q \hat{d}
\end{aligned}
$$

where

$$
\begin{aligned}
& P=\left(A_{1}-A_{2}\right) X+\left(B_{1}-B_{2}\right) U \\
& Q=\left(C_{1}-C_{2}\right) X+\left(E_{1}-E_{2}\right) U
\end{aligned}
$$

By collecting the dc terms from Eq.(3.10), the steady state solutions are

$$
\begin{gathered}
X=-A^{-1} B U \\
Y=\left(-C A^{-1} B+E\right) U
\end{gathered}
$$

The small signal solutions can be obtained by taking the Laplace transform of $\mathrm{Eq} \cdot(3.13)$.

$$
\begin{aligned}
& \hat{x}=H_{u x} \hat{u}+H_{d x} \hat{d} \\
& \hat{y}=H_{u y} \hat{u}+H_{d y} \hat{d}
\end{aligned}
$$


where

$$
\begin{aligned}
& \left.\frac{\hat{x}}{\hat{u}}\right|_{\hat{d}=0}=H_{u x}=(s I-A)^{-1} B \\
& \left.\frac{\hat{x}}{\hat{d}}\right|_{\hat{u}=0}=H_{d x}=(s I-A)^{-1} P \\
& \left.\frac{\hat{y}}{\hat{u}}\right|_{\hat{d}=0}=H_{u y}=C H_{u x}+E \\
& \frac{\hat{y}}{\left.\frac{\hat{d}}{~}\right|_{\hat{u}=0}=H_{d y}=C H_{d x}+Q}
\end{aligned}
$$

Eqs.(3.16 - 3.17) describe the power stage of switching converters with unterminated input and output. The differences between the above model and the conventional models for the load terminated case are illustrated in the following example.

Example: Let us consider a boost converter, as shown in Fig.3.4. For simplicity, let us assume the switch is ideal and $R_{c}=0, R_{\ell}=0$. The two switched models are as shown in Fig.3.5. For choice of state vector $x^{T}=\left[\begin{array}{lll}i_{1} & v_{2}\end{array}\right]$, the state equations become:

$$
\begin{gathered}
\text { i) interval } d_{1} T_{s} \\
A_{1}=0, B_{1}=\left[\begin{array}{cc}
-\frac{1}{L} & 0 \\
0 & \frac{1}{C}
\end{array}\right] \quad A_{2}=\left[\begin{array}{rr}
0 & \frac{1}{L} \\
-\frac{1}{C} & 0
\end{array}\right], B_{2}=\left[\begin{array}{rr}
-\frac{1}{L} & 0 \\
0 & \frac{1}{C}
\end{array}\right]
\end{gathered}
$$




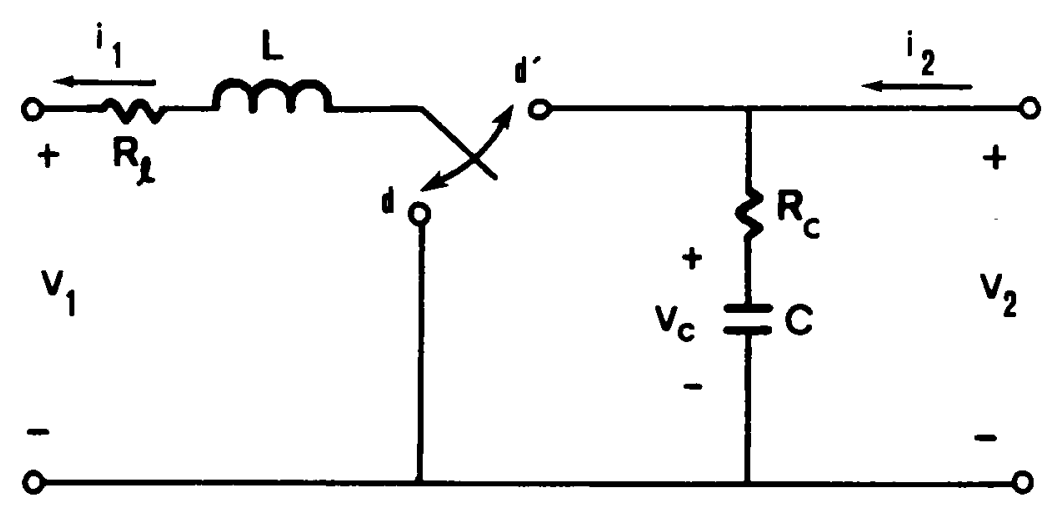

Figure 3.4 Boost converter power stage

i) interval $d T_{S}$

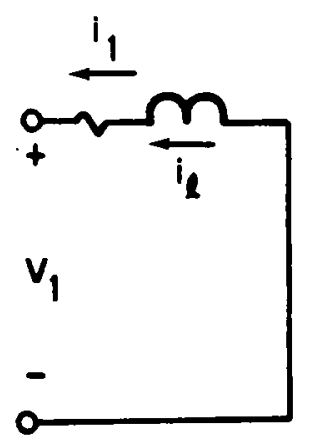

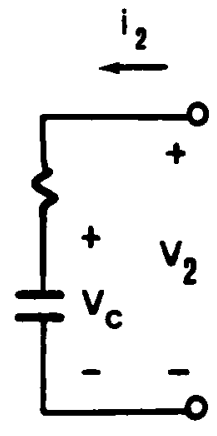

ii) interval $d^{\prime} T_{s}$

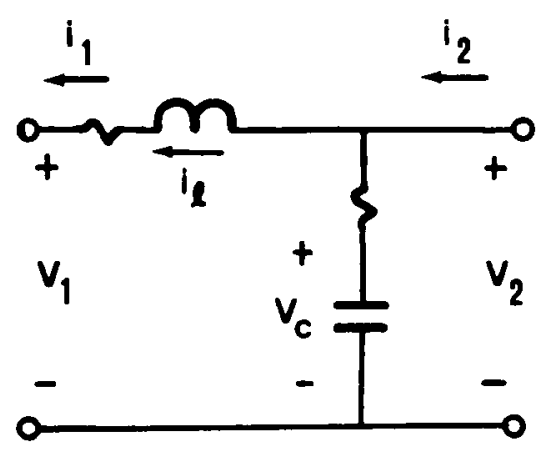

Figure 3.5 Two switched circuit models of the circuit in Fig.3.4 


$$
C_{1}=C_{2}=I, \quad E_{1}=E_{2}=0
$$

(Note that, for simplicity, the sign convention of the states and outputs are purposely chosen so that the states are identical to outputs.)

From Eq.(3.11) the averaged power stage coefficient matrices are

$$
A=\left[\begin{array}{cc}
0 & \frac{D^{\prime}}{L} \\
-\frac{D^{\prime}}{C} & 0
\end{array}\right], \quad B=\left[\begin{array}{cc}
-\frac{1}{L} & 0 \\
0 & \frac{1}{C}
\end{array}\right], \quad C=I
$$

Using Eq.(3.15), the steady state solution is

$$
Y=X=\left[\begin{array}{l}
I_{1} \\
V_{2}
\end{array}\right]=\frac{1}{D^{\prime}}\left[\begin{array}{l}
I_{2} \\
V_{1}
\end{array}\right]
$$

The control coefficient matrices using Eq.(3.14) become

$$
P=\left[\begin{array}{c}
-\frac{V_{1}}{D^{\prime} L} \\
\frac{I_{2}}{D^{\prime} C}
\end{array}\right], \quad Q=0
$$

The small signal model for the power stage is obtained from Eqs.(3.18) through (3.21) and using (3.23) through (3.25) results in 


$$
\begin{aligned}
& H_{u y}=\frac{\hat{y}}{\hat{u}}=\frac{1}{\Delta}\left[\begin{array}{ccc}
-\frac{C}{D^{\prime 2}} & \frac{1}{D^{\prime}} \\
\frac{1}{D^{\prime}} & \frac{L}{D^{\prime 2}} s
\end{array}\right] \\
& H_{d y}=\frac{\hat{y}}{\hat{d}}=\frac{1}{\Delta}\left[\begin{array}{c}
\frac{I_{2}}{D^{\prime 2}}\left(1-\frac{C V_{1}}{D^{\prime} I_{2}} s\right) \\
\frac{V_{1}}{D^{\prime 2}}\left(1+\frac{L I_{2}}{D^{\prime} V_{1}} s\right)
\end{array}\right]
\end{aligned}
$$

where

$$
\Delta=1+s^{2} / \omega_{0}^{2}, \quad w_{0}=D^{\prime} / \sqrt{L C}
$$

It is noticed that the open loop transfer functions in Eq. (3.26) and (3.27) have infinite Q (with ideal elements), which clearly implies the unterminated system. However, the control to output transfer function in Eq. (3.27) carries the dc input information, $V_{1}$ and $I_{2}$. This is due to earlier assumption that the model describes the behavior of the regulator about a known steady state operating condition. From the statespace averaged model, the A-matrix does not carry steady state information while the control matrix $P$ does. This may be seen more clearly in the linear equivalent canonical circuit model in Fig.3.6 which can be generated either from the state-space equations or from the circuit averaging 


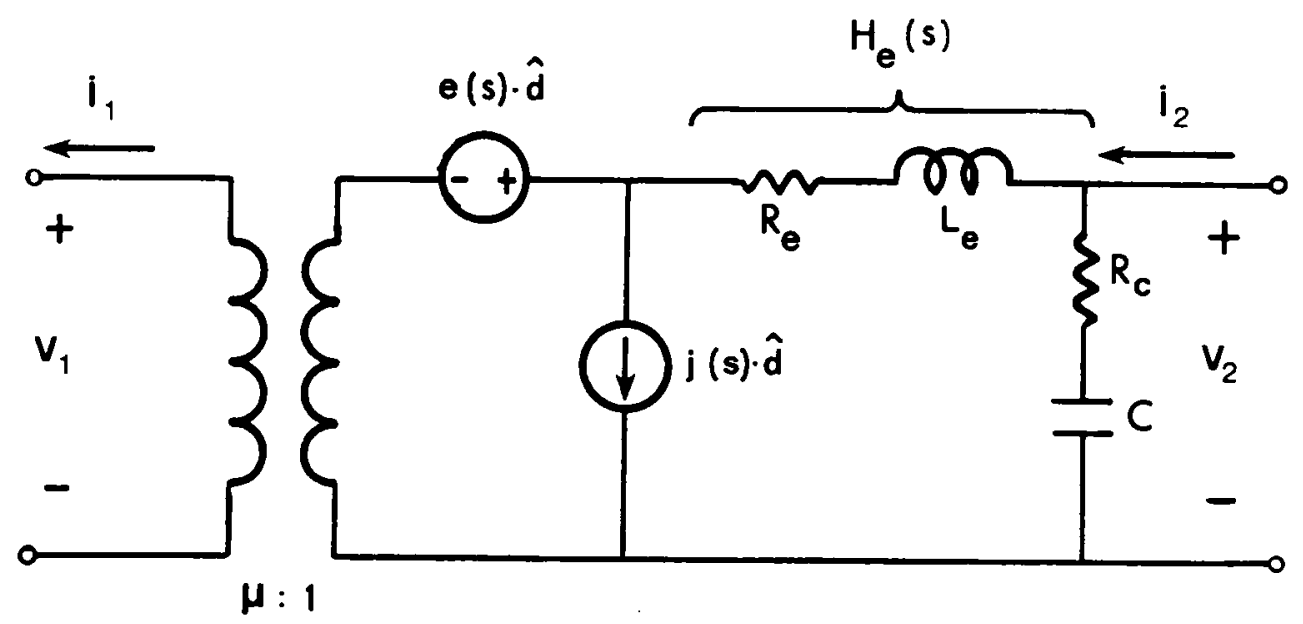

For the boost converter,

$$
\begin{gathered}
L_{e}=\frac{L}{D^{\prime 2}}, \quad R_{e}=\frac{R_{\ell}}{D^{\prime 2}}, \mu=D^{\prime} \\
e(s)=\frac{V_{1}}{D^{\prime 2}}\left(1+\frac{L}{D^{\prime}} \cdot \frac{I_{2}}{V_{1}} s\right)=\frac{V_{1}}{D^{\prime 2}}\left(1-\frac{L}{D^{\prime 2} R_{L}} s\right) \\
j(s)=\frac{I_{2}}{D^{\prime}}=\frac{-V_{2}}{D^{\prime} R_{L}}
\end{gathered}
$$

where

$$
R_{L}=-\frac{V_{2}}{I_{2}} \text { or }\left.Z_{L}(s)\right|_{s \rightarrow 0}
$$

Figure 3.6 Linear equivalent canonical circuit model of the switching converter power stage 
technique [3]. In the circuit model the control dependent sources e(s) and $j(s)$ are functions of the steady state input values while the effective filter transfer function, $H_{e}(s)$, is still unterminated. Hence, if a regulator is terminated with a resistive load $R_{L}$, the same $R_{L}$ would appear in $e(s), j(s)$ and $H_{e}(s)$. However, if a regulator is terminated with a frequency dependent load $Z_{L}$, only the steady state dc value of $Z_{L}$ (i.e., $R_{L}$ or $V_{2} / I_{2}$ ) appears in $e(s)$ and $j(s)$ while $H_{e}(s)$ is terminated with $Z_{L}$.

This can be shown from the load terminated open loop transfer functions. For instance, the input voltage to output voltage transfer function becomes

$$
\mathrm{H}_{\mathrm{vL}}=\frac{\hat{\mathrm{v}}_{2}}{\hat{\mathrm{v}}_{1}} \mid \hat{\mathrm{d}=0, \mathrm{Z}_{\mathrm{L}}}=\frac{1}{\mathrm{D}^{\prime}} \cdot \frac{1}{\left(1+\frac{\mathrm{L}}{\mathrm{D}^{\prime 2} \mathrm{Z}_{\mathrm{L}}} \mathrm{s}+\frac{\mathrm{s}^{2}}{\omega_{\mathrm{O}}^{2}}\right)} \equiv \frac{1}{\mathrm{D}^{\prime}} \mathrm{H}_{\mathrm{e}}(\mathrm{s})
$$

and the control to output voltage transfer function is

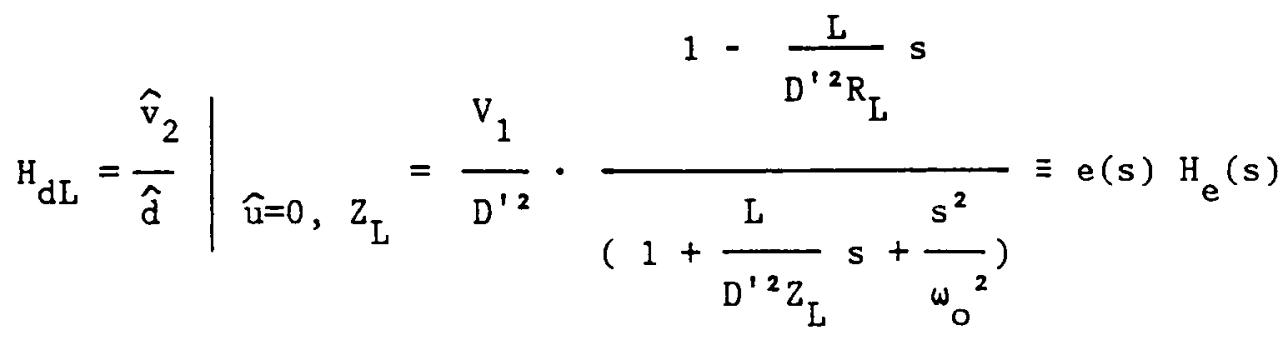

where

$$
R_{L}=-V_{2} / I_{2}
$$


The analysis of the complete regulator using the above results is presented in Section 3.4 .

\subsubsection{Analog feedback controller}

The commonly used analog feedback controller is basically linear for normal modes of operations. The controller can be modeled by either transfer functions or state equations.

The transfer function model, in Eq. (3.31) provides more design insight so that both analysis and synthesis of the controller can be done quite easily. Pole-zero compensations of the closed loop system can be directly implemented using the Bode plot technique.

$$
F(s)=\frac{\omega_{m}}{s} \cdot \frac{\left(1+s / \omega_{21}\right)\left(1+s / \omega_{22}\right)}{\left(1+s / \omega_{p 1}\right)}
$$

However, the second order effects of the feedback, such as the steady state switching ripple component, is lost.

Modeling with state equations can describe the circuit in more detail, and can easily include the nonlinearities such as op-amp saturation, soft starter and protection circuits. However, the circuit topology must be pre-fixed and generally, the model requires more memory space. Also, the model does not provide direct information about the location of poles and zeros to facilitate design of feedback compensator. 


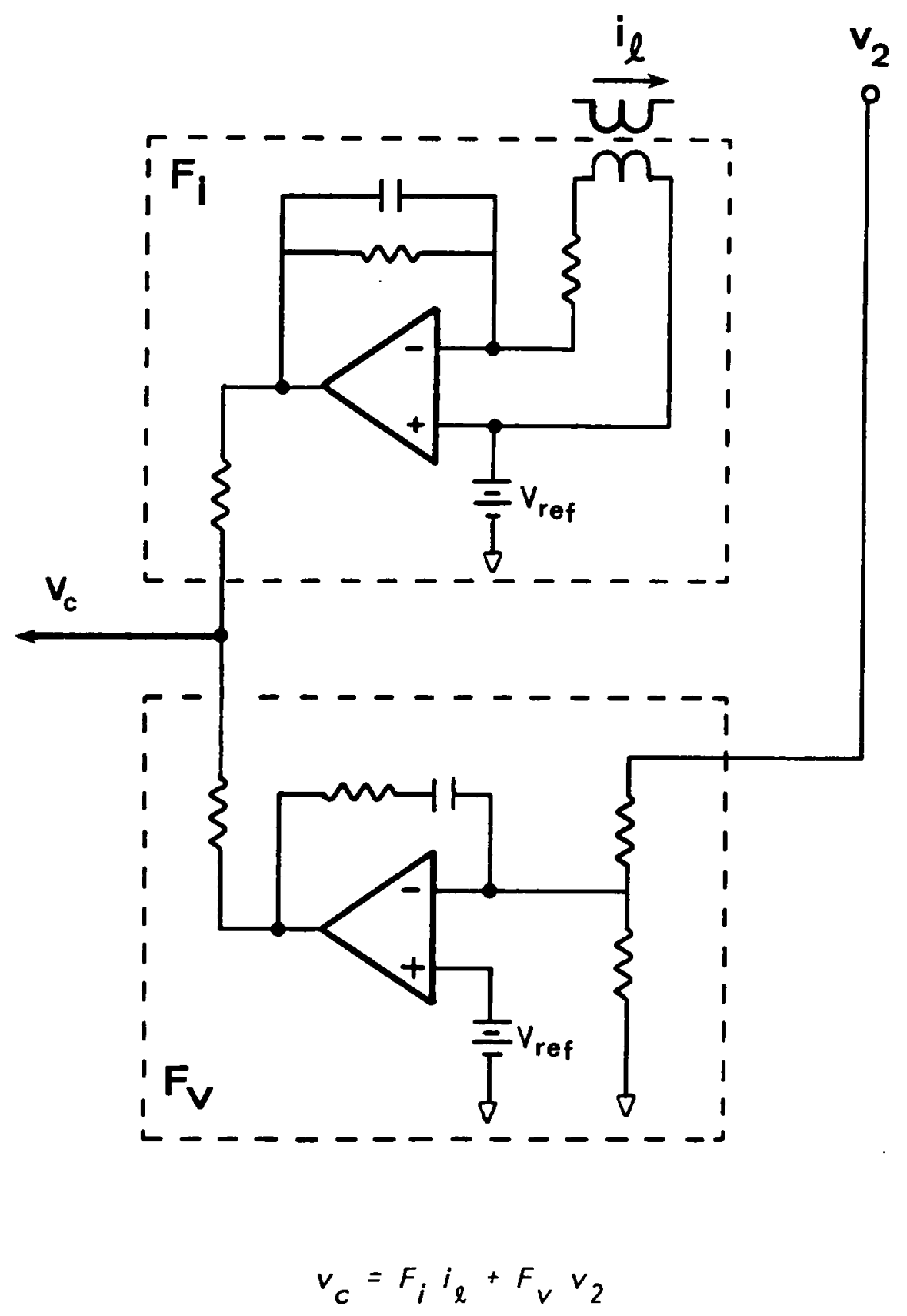

Figure 3.7 A feedback compensator (two-loop) 
The choice of model depends on its objective. For design and frequency domain analysis the transfer function model suits better; and for more accurate time-domain analysis and simulation the state equation model may be the better choice. The model developed for this study includes both modeling techniques.

Fig.3.7 illustrates a typical multiple loop controller for switching regulators. The controller senses the state (inductor current) and output (output voltage). The sum of the state and output compensators $\left(F_{x}\right.$ and $F_{Y}$ ) is the control voltage, $v_{c}$, as described in Eq. (3.32).

$$
v_{c}=-\left(F_{x} x+F_{y} y\right)
$$

The compensators for a regulator employing the inductor (or switch) current and output voltage sensed feedback become

$$
F_{x}=\left[\begin{array}{ll}
F_{i} & 0
\end{array}\right], \quad F_{y}=\left[\begin{array}{ll}
0 & F_{v}
\end{array}\right]
$$

\subsubsection{Digital Signal Processor}

The digital signal processor (DSP) converts the output control signal $v_{c}$ from the analog feedback controller into discrete-time pulses to control the ON-OFF of the power switch. The DSP includes the threshold detector (comparator), the ramp function and the latch circuit. 
The threshold detector compares the input control signal, $v_{c}$, and the reference signal (either dc or the ramp) to generate the trigger signal for the transistor OFF command. The latch circuit then generates the PWM signal, $d(t)$, as shown in Fig.3.2 to the power transistor. For the multiple loop control, the input control signal, $v_{c}$, is the sum of the outputs of each feedback loop. In this case $v_{c}$ may include the ramp function (from the inductor current or switch current feedback), then the external ramp function can be optional, which quite often is needed to stabilize the system operating more than $50^{\circ}$ duty ratio. Fig.3.8 illustrates this multiple loop case. For each different duty ratio control law such as constant frequency, constant $T_{\text {on }}$ and constant $T_{\text {off }}$, the DSP model should be different. These different modules are stored in the component module library, as in the case of power stage models.

For the small signal model, the transfer function from the control signal $v_{c}$ to the duty cycle modulation $d$ is obtained as in Eq.(3.34) using the describing function technique.

$$
\mathrm{d}=\mathrm{FM} \cdot \mathrm{v}_{\mathrm{c}}
$$

\subsubsection{Switching regulator small signal modeling and analysis}

Connecting the power stage, analog feedback controller and the digital controller (Figs. 3.6, 3.7 and 3.8) together completes the small signal regulator model as shown in Fig.3.9. This unterminated regulator is ready 


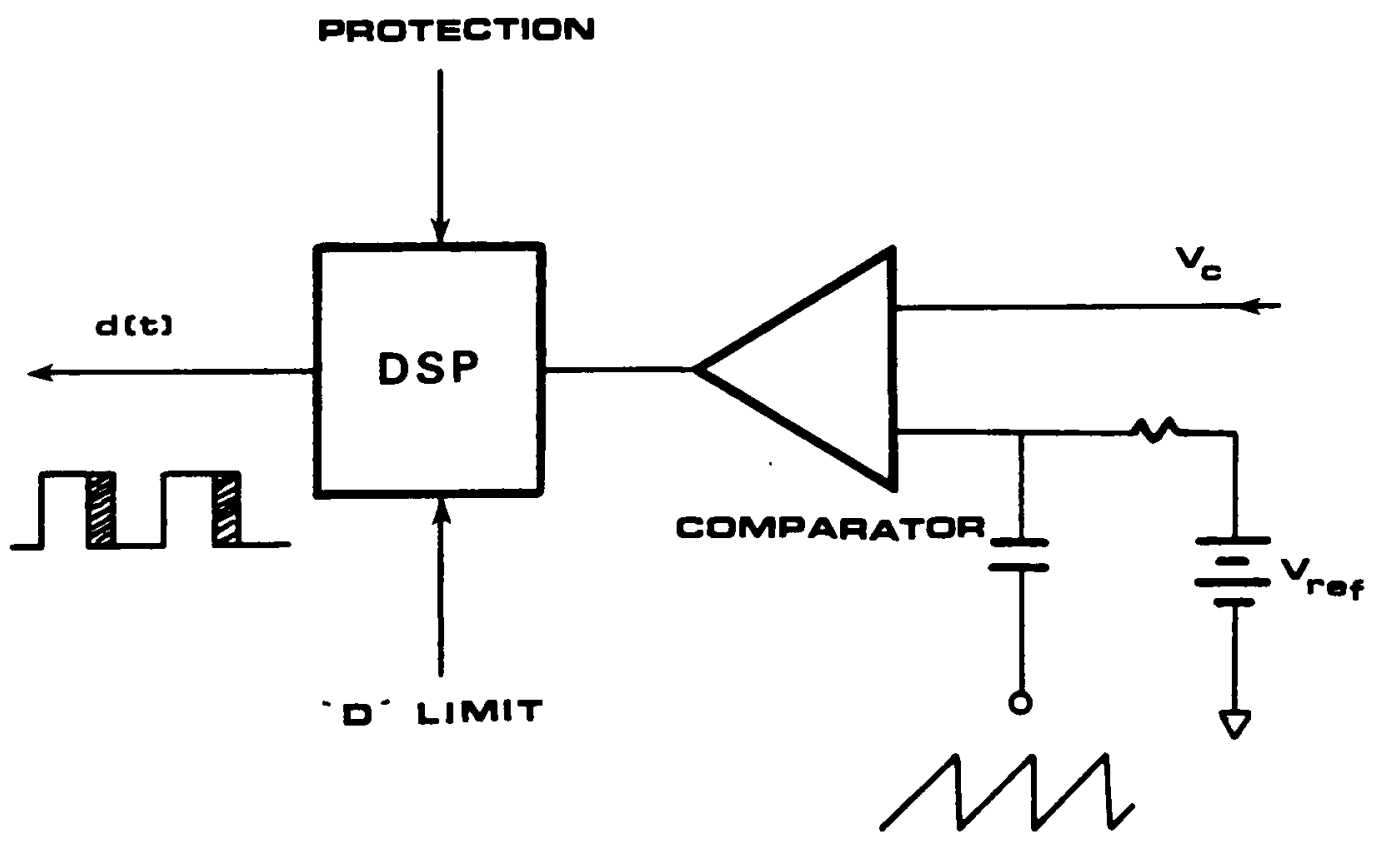

Figure 3.8 Pulse-width-modulator (PWM) 
to be connected into a system. Analysis of the doubly terminated regulator can be carried out using the g-parameters of the regulator. The g-parameters can be derived either from the circuit model shown in Fig. 3.9 or from the block diagram representation in Fig.3.10. The block diagram model in Fig.3.10 which uses transfer functions can describe any type of converter topology and associated controls. While the model shown in Fig.3.9 is circuit oriented, and it does provide physical insight into the system.

The closed loop transfer functions from the the input vector $\hat{u}$ to the output vector $\hat{y}$, the G-matrix of the regulator, can be directly derived from the transfer function model in Fig.3.10.

$$
\frac{\hat{y}}{\hat{u}}=\left[I+T_{e q}\right]^{-1} \cdot\left[H_{u y}-F M \cdot H_{d y} \cdot F_{x} \cdot\left[\left[I+T_{x}\right]^{-1} \cdot H_{u x}\right]\right]
$$

where

$$
\begin{gathered}
T_{e q}=F M \cdot H_{d y} \cdot\left[F_{y}-F_{x} \cdot\left[\left[I+T_{x}\right]^{-1} \cdot H_{d x} \cdot F_{y} \cdot F M\right]\right] \\
T_{x}=H_{d x} \cdot F_{x} \cdot F M
\end{gathered}
$$

Using the g-parameters in Eq. (3.35), performance parameters of the regulator operating in the system such as stability, audiosusceptibility, input and output impedances etc. can be derived as functions of impedances of its terminating subsystems. 


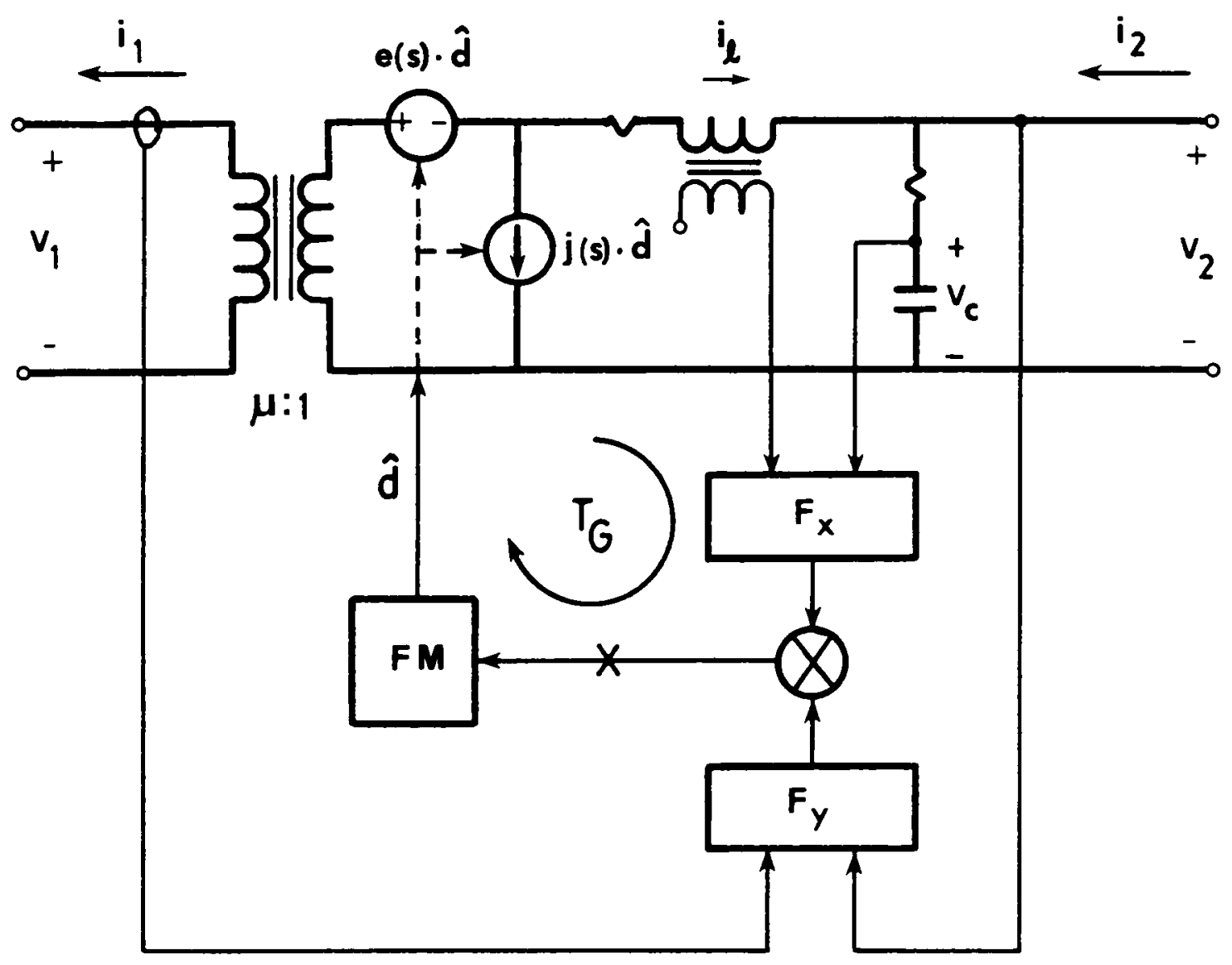

Figure 3.9 Small signal model of switching regulators 


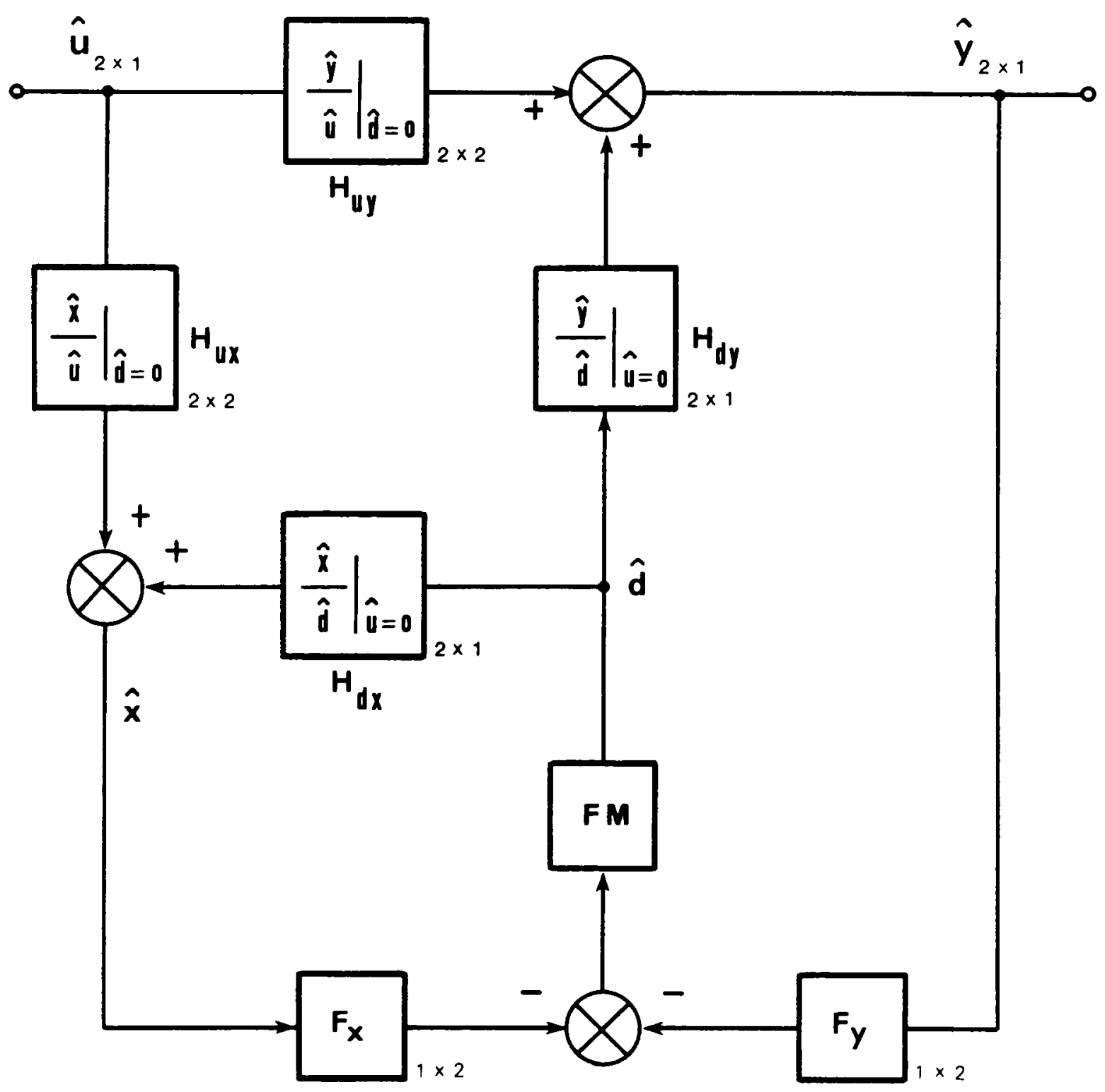

Figure 3.10 Small signal transfer function model of switching regulator 
Analysis of a complete regulator is illustrated in the following example using the boost converter power stage derived in Section 3.2.1. For simplicity, a single loop (output voltage feedback) control is used.

Example: Analysis of the boost regulator using the g-parameters

For single loop output feedback control, the compensators are

$$
F_{x}=0, \quad F_{y}=\left[\begin{array}{ll}
0 & F_{v}
\end{array}\right]
$$

and let the PWM gain be FM.

The closed loop input to output transfer functions in Eq. (3.35) becomes

$$
G=\left[I+F M H_{d y} F_{y}\right]^{-1} H_{u y}
$$

Using the results of the power stage equations in Section 3.2 .1 (Eqs. (3.26) and (3.27)), the g-parameters in Eq. (3.39) are

$$
\begin{aligned}
& g_{11}=-\frac{1}{\Delta\left(1+T_{G}\right) D^{\prime 2}}\left[\left(1+T_{G}\right) C s+\frac{F M}{\Delta} \cdot \frac{I_{2}}{D^{\prime}}\left(1-C \frac{V_{1}}{I_{2}} s\right) F_{v}\right] \\
& g_{12}=\frac{1}{\Delta\left(1+T_{G}\right) D^{\prime}}\left[\left(1+T_{G}\right) \frac{1}{D^{\prime}}-\frac{F M}{\Delta} \cdot \frac{I_{2}}{D^{\prime 3}} L(1-C-s) F_{v}\right]
\end{aligned}
$$




$$
\begin{aligned}
& g_{21}=\frac{1}{\Delta\left(1+T_{G}\right)} \frac{1}{D^{\prime}} \\
& g_{21}=\frac{1}{\Delta\left(1+T_{G}\right)} \frac{L}{D^{\prime 2}} s
\end{aligned}
$$

where

$$
\begin{gathered}
\Delta=1+s^{2} / \omega_{0}^{2} \\
T_{G}=\frac{F M V_{1}}{\Delta D^{\prime 2}}\left(1+\frac{L}{D^{\prime} V_{1}} s\right) F_{V}
\end{gathered}
$$

Here, $1 / \Delta$ is the effective filter transfer function $H_{e}(s) . T_{G}$ is the loop gain defined at Point $X$ for the unterminated regulator shown in Fig.3.9. The stability of the unterminated regulator can be determined using the quantity $T_{G}$. The Bode plot technique can be used to check the Nyquist stability criterion. Using Eqs. (3.40) through (3.45), the total system can be treated as a doubly terminated two-port system focusing on the switching regulator. The system analysis can then be carried out by defining the source and load impedances seen by the regulator.

As described in Section 2.4, a doubly terminated regulator (two-port) can be analyzed in two-steps. First, by considering the load terminated regulator (one-port) with ideal source and then including the source impedance $Z_{S}$. These steps when compared to the expression for a doubly terminated two-port system in Eqs.(2.3) and (2.4), provide more physical insight into the system analysis especially for the interactions from 
either the source or the load to the regulator.

\section{Singly (load) terminated regulator:}

Suppose that the regulator is terminated by a load impedance of $Z_{L}$, which is frequency dependent with a dc value of $R_{L}$, as shown in Fig.3.11. The line transfer function from $\hat{v}_{1}$ to $\hat{v}_{2}$ is

$$
G_{v L}=\frac{\hat{v}_{2}}{\hat{v}_{1}}=\frac{g_{21}}{1+g_{22} / Z_{L}} \equiv \frac{g_{21}}{1+T_{L}}
$$

where the values of $g$-parameters are in Eqs.(3.40) through (3.43).

Before we analyze Eq.(3.46), let us expand this equation for comparison with the regulator terminated with resistive load $R_{L}$. Knowing the steady state operating condition (i.e., dc output power), the steady state input values can be expressed in terms of the regulated dc output voltage and the dc output power.

$$
\mathrm{V}_{1}=\mathrm{V}_{2} / \mathrm{D}^{\prime}, \mathrm{I}_{2}=-\mathrm{V}_{2} / \mathrm{R}_{\mathrm{L}}
$$

where

$$
R_{L} \equiv V_{2}^{2} / P_{\text {out }}
$$

Expanding Eq.(3.46), the line voltage transfer function becomes 


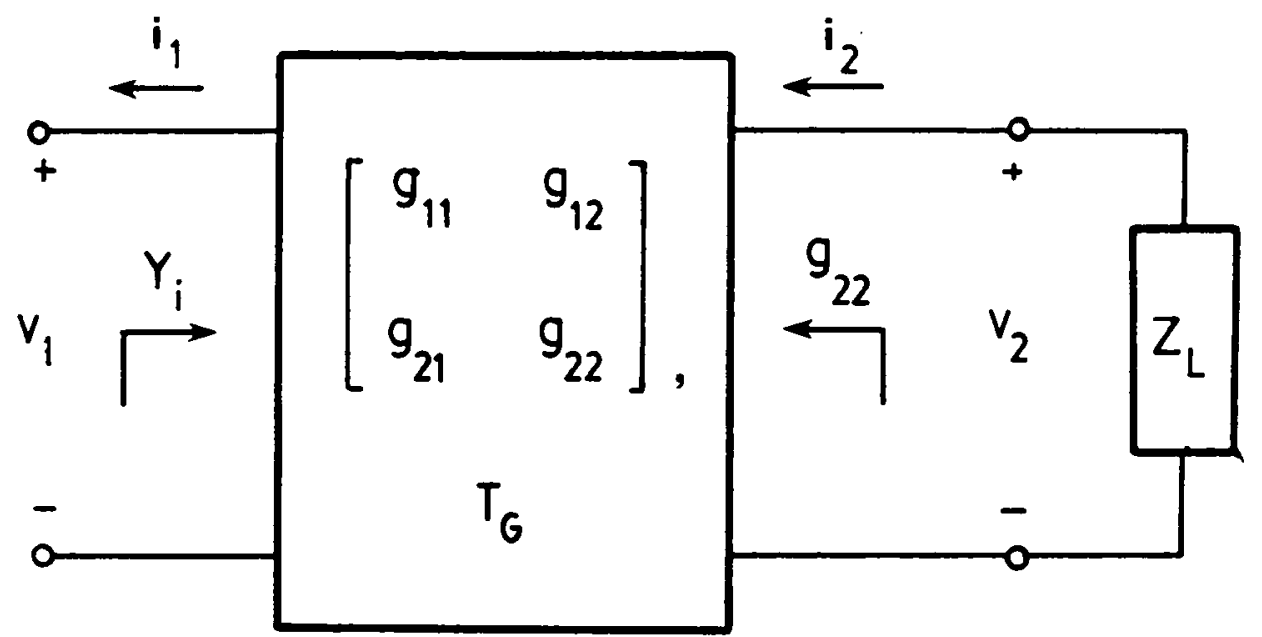

Figure 3.11 Load terminated switching regulator 


$$
\frac{\hat{v}_{2}}{\hat{v}_{1}}=\frac{1}{D^{\prime}} \frac{1}{\left[1+\frac{L}{D^{\prime 2} Z_{L}} s+\frac{s^{2}}{\omega_{0}^{2}}\right]+\left[\frac{V_{2}}{D^{\prime}}\left(1-\frac{L}{D^{\prime 2} R_{L}} s\right) F M F_{V}\right]}
$$

It should be noted that in Eq. (3.48) the term (1) includes $Z_{L}$ and the term (2) includes $R_{L}$. This again can be seen clearly from Fig.3.6 where the effective filter in the power stage model (the term (1)) is now terminated with $Z_{L}$, while the control source is a function of the dc operating condition (the term (2)).

If the load impedance, $Z_{L}$, is complex frequency dependent, it is neither easy to obtain design insight nor to analytically determine the stability of the regulator using Eq.(3.48). However, in Eq. (3.46), $Z_{L}$ is factored out so that the stability of the regulator can be determined using the quantity $\mathrm{T}_{\mathrm{L}}$. The load interaction criteria of the regulator can also be examined from $T_{L}$. For instance, if one assumes that $g_{21}$ or $\mathbf{g}_{22}$ is stable, then the Nyquist stability condition is simplified such that the regulator is stable if $T_{L}$ does not encircle the point $(-1,0)$. The condition can easily be checked using the Bode plot technique. Furthermore, under the above assumptions the sufficient condition for stability is simply $\left|g_{22}\right|<\left|Z_{L}\right|$ for all frequency.

Using Eq.(2.5), the input impedance $Z_{i}=1 / Y_{i}$ of the load terminated regulator shown in Fig.3.11 can be derived as 


$$
Y_{i}=-\frac{g_{11}+\Delta G / Z_{L}}{1+g_{22} / Z_{L}} \equiv \frac{Y_{\text {eq }}}{1+T_{L}}
$$

The stability of the regulator can also be determined from Eq. (3.49) using $\mathrm{T}_{\mathrm{L}}$. Analysis of the doubly terminated regulator taking into account of the source interactions can be carried out using $Y_{i}$. Eq. (3.49) can be expanded done as in the line transfer function in Eq.(3.48). At low frequencies where $\Delta G$ and $g_{22}$ are vanishingly small and $Y_{i}$ becomes $-\left.g_{11}\right|_{s \rightarrow 0}$, the input impedance characteristic is

$$
Y_{i}=-\frac{1}{D^{\prime 2} R_{L}}
$$

This exhibits the negative input resistance characteristic as expected for all switching regulators.

\section{Doubly terminated regulator:}

Fig.3.12 shows the complete (doubly terminated) regulator system. The performance parameters of the system are derived using the results from the load terminated regulator as follows: first, the two line transfer functions defined in Fig.3.12 are expressed as

$$
G_{s 1}=\frac{\hat{v}_{2}}{\hat{v}_{s}}=\frac{z_{s}}{z_{s}+z_{i}}=\frac{1}{1+z_{s} / z_{i}} \equiv \frac{1}{1+T_{s}}
$$




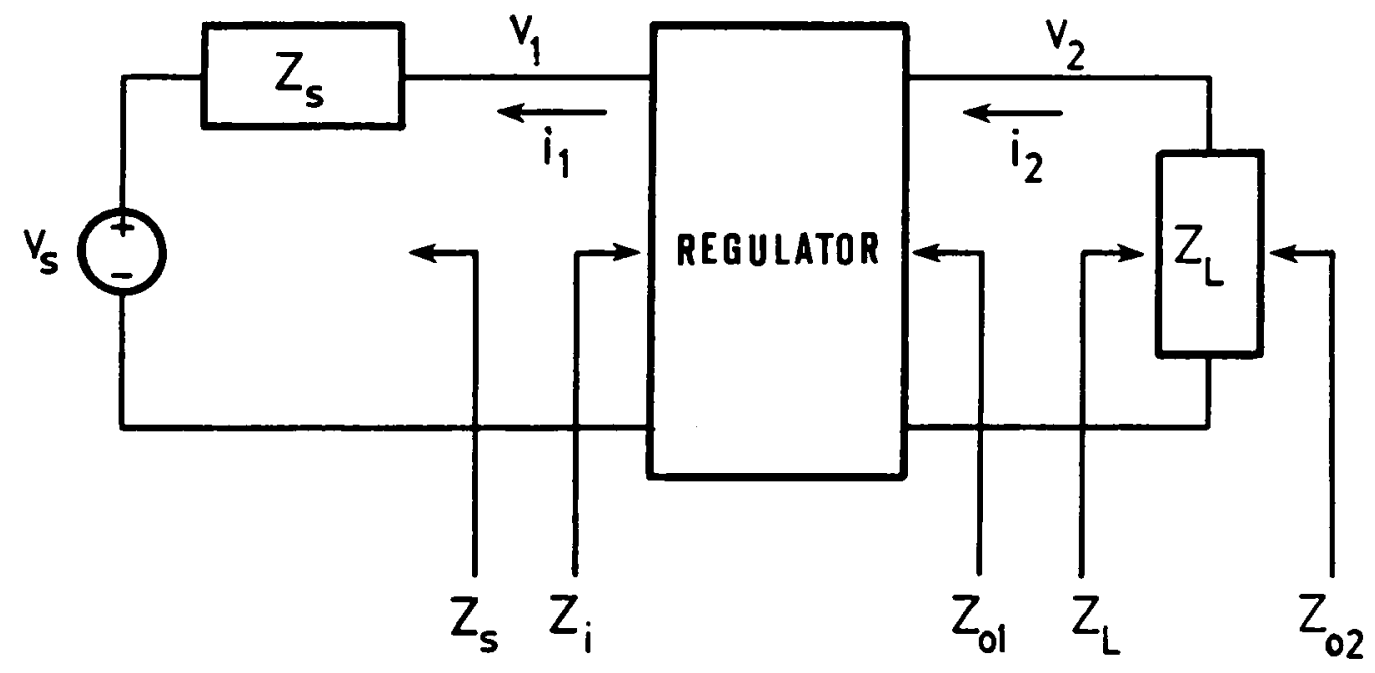

Figure 3.12 Doubly terminated switching regulator 


$$
G_{s 2}=\frac{\hat{v}_{2}}{\hat{v}_{s}}=\frac{v_{1}}{v_{s}} \cdot \frac{v_{2}}{v_{1}}=G_{s 1} \cdot G_{v L}
$$

where $G_{v L}$ is in Eq. $(3.46)$

From Eqs. (3.51) and (3.52), the stability of the doubly terminated regulator can be determined using $T_{S}$. If $G_{v L}$ is stable, which implies $Y_{i}$ does not have RHP poles, then the Nyquist stability condition can be checked the by Bode plots of $\mathrm{T}_{\mathrm{S}}$. Also, the source interactions to the regulator can be examined using $\mathrm{T}_{\mathrm{S}}$.

Secondly, the output impedances of the regulator can be defined as $Z_{01}$ and $Z_{\circ 2}$ as shown in the figure. $Z_{\circ 1}$ is used for analyzing the interactions between the regulator terminated with $Z_{s}$ and the external load. $\mathrm{Z}_{\circ 2}$ is used to characterize the regulator behavior due to the load disturbance, such as transient analysis due to a step load.

$$
\begin{gathered}
Z_{\circ 1}=\frac{g_{22}-\Delta G Z_{s}}{1-g_{11} Z_{s}} \\
Z_{\circ 2}=z_{\circ 1} \| Z_{L}
\end{gathered}
$$

The analysis for the boost regulator example shows that, using the g-parameters, singly or doubly terminated regulator performance parameters can easily be obtained along with the interaction information between 
the regulator and its source and load. The analysis can be applied to any two-port subsystem. Thus, one can focus on a particular subsystem and its interactions with the remainder of a total system. Some examples analyzing the source and load interactions of switching regulators are discussed in detail in Chapter 6. 


\subsection{Solar Array Modeling}

Solar arrays made of silicon solar cells are the main source of electrical power for spacecraft power systems. Because the individual silicon cell used on these arrays is small and produce very little power, many solar cells are connected in series and parallel combination to provide necessary power to the spacecraft.

Several approaches have been proposed for modeling a solar array [6]. First, it is possible to model an array by an interconnection of individual solar cells (individual cell model approach). Advantages of this approach are: the parameters for the individual cell model are readily obtained using established measurement and calculation procedures, also the effects of parameter variation can be easily included. Shadowing effects as well as cell faults can easily be inserted into the array. However, the major disadvantage of this approach is that an extremely large amount of computation time is required, which might easily exceed the total computer capacity.

Another approach is to use a single cell model to simulate the entire array (macro model approach). The major advantage to this approach is its ability to minimize the computer time and memory space needed to analyze array behavior. Parameters can be obtained by incorporating the measurement/calculation of the individual cells and their interconnection scheme, or simply by making terminal measurements of the array itself. Neither of these methods takes much more effort than would be needed for the individual cell model. One disadvantage to the macro model approach 
is the fact that a new set of macro model parameters needs to be developed for each different array configuration. This tends to make the model less flexible than is desired. Furthermore, shadowing and individual cell faults cannot be easily included in the macro model without, again, a redevelopment of model parameters.

An approach which uses a combination of the macro model and the individual cell model could be very advantageous. This approach retains the flexibility of the individual cell model approach while reducing the necessary computer time and capacity. Any fault that occurs in an individual cell can be simulated. Shadowing effects can be accounted for in much the same way as would be done in the individual model. Sensitivity of parameters can also be easily analyzed by varying individual cell parameters. The disadvantage of using the combination model is the need to determine at least two sets of model parameters -- one set for the group of individual cells and one set for the group of macro models.

The choice of modeling approaches depends on the particular modeling objective(s). In the present study, the macro model approach shown in Fig.3.13 is used for performing an overall system analysis. With this type of analysis it is presumed that the model of the solar array need not have great detail.

\subsubsection{Solar array DC model}

For this study, two types of DC models are generated. One is the analytical macro model and the other is the empirical data model. For the 


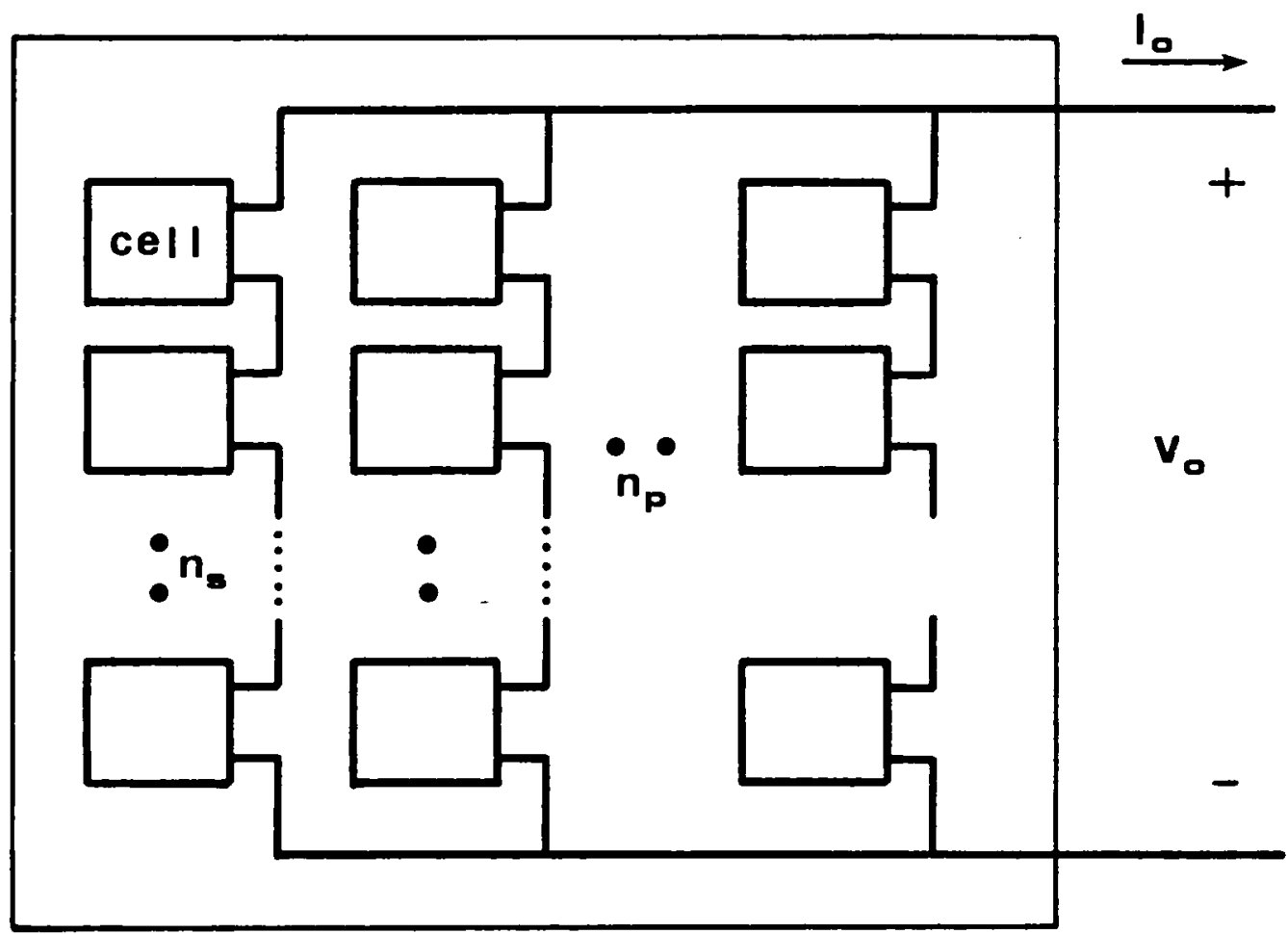

Figure 3.13 Solar array macro modeling 
analytical solar array macro model, the standard solar cell DC model shown in Fig.3.14 is used [7]. It provides very good first order predictions of the DC behavior of the silicon cell. A great deal of literature is available for determining its parameter values. An analytical expression that describes the terminal characteristics of the solar cell model in Fig. 3.14 is

$$
I_{0}=I_{s}-I_{\text {do }}\left[\exp \left(\frac{Q V_{D}}{K T}\right)-1\right]-\frac{V_{D}}{R_{s h}}
$$

Based on the cell model, a solar array macro model is constructed by assembling solar cells in parallel and series connections as shown in Fig.3.13. In Fig.3.15, the resulting equivalent circuit model is illustrated where its elements are lumped parameters and are functions of the number of cells in series, $n_{s}$, and the number of parallel strings, $n_{p}$. The analytical expression for the terminal I-V characteristic of the solar array model in Fig. 3.15 is then

$$
I_{0}=n_{p}\left[I_{s}-I_{d o}\left[\exp \left(\frac{Q V_{D}}{K T}\right)-1\right]-\frac{V_{D}}{R_{s h}}\right]
$$

where

$$
V_{D}=\frac{V_{0}}{n_{s}}+\frac{R_{s} \cdot I_{0}}{n_{p}}
$$

From Eqs. (3.56) and (3.57) the solar array operating conditions are set by the load power demand and shunt regulator limiting voltage. Other more elaborate analytical DC models exist, such as multiple element models 


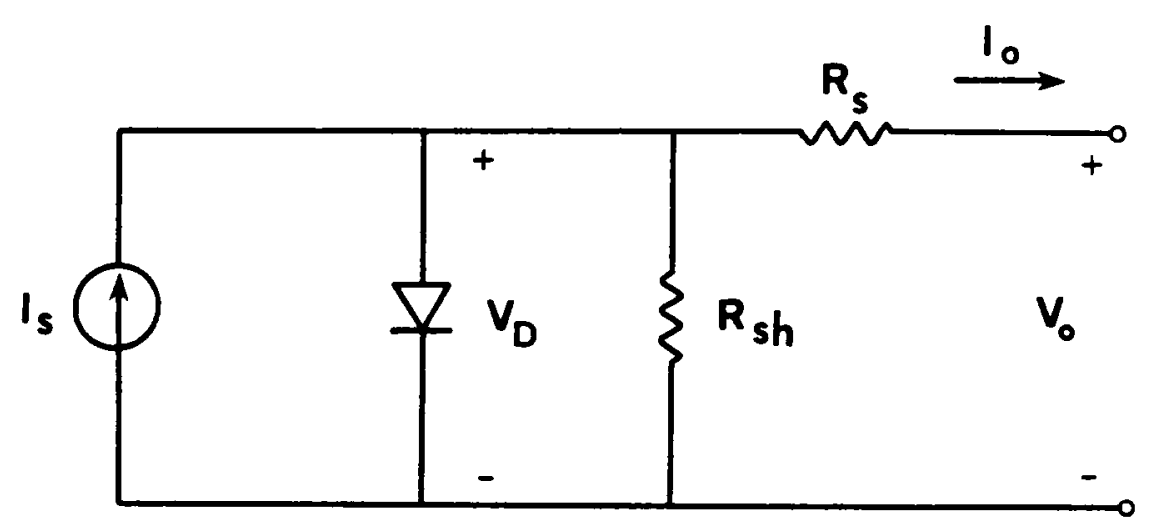

Figure $3.14 D C$ model of solar cell

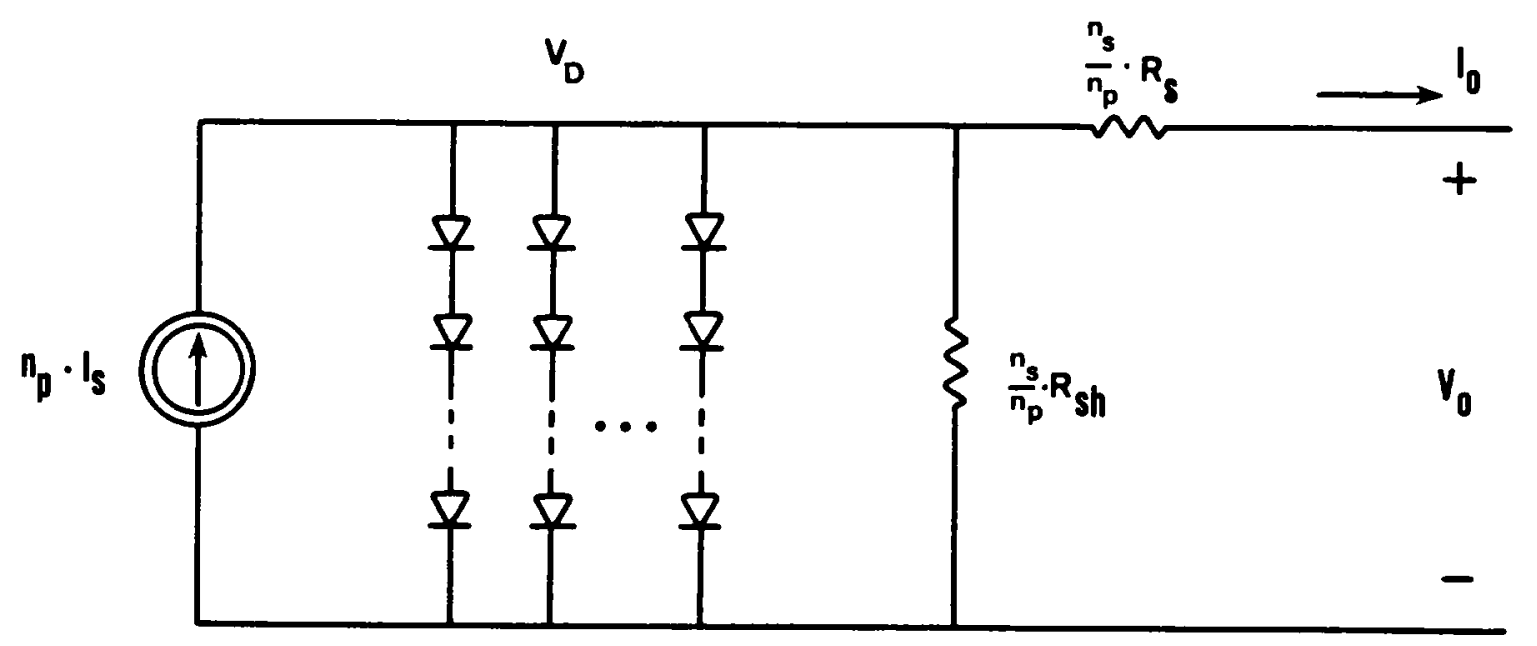

Figure 3.15 DC macro model of solar array 
which include second order effects [8]. Those multiple-element models are used to model accurately the effects of distributed series resistance, complex voltage dependencies and radiation. Due to the complexities of the model it is not practical to incorporate these second order effects in the system study.

The other model generated for this study is based on the empirical data about the solar array terminal I-V characteristics. The solar array illumination level and temperature are chosen as the dependent variables. Based on four dimensional empirical data, both linear and high order interpolation techniques are employed to define the array $I-V$ characteristic curve for any arbitrary temperature and illumination. Fig.3.16 shows the interpolated I-V characteristics based on two interpolated empirical data curves for two different illumination levels. In these data, temperature is assumed constant. Other dependent variables for the array characteristics can be added, provided their effects can be accounted for by the measurement. If the measurement data is available, this approach is simple and accurate.

The solar array dc output resistance, $r_{o}$ is the tangential slope of its terminal $I-V$ characteristic.

$$
r_{0}=-\left.\frac{\Delta V}{\Delta I}\right|_{I_{0}, V_{0}}
$$

$r_{0}$ can be also analytically derived by replacing the diode in the solar array dc model in Fig. 3.15 by the nonlinear diode dynamic resistance $\mathrm{r}_{\mathrm{d}}$. 


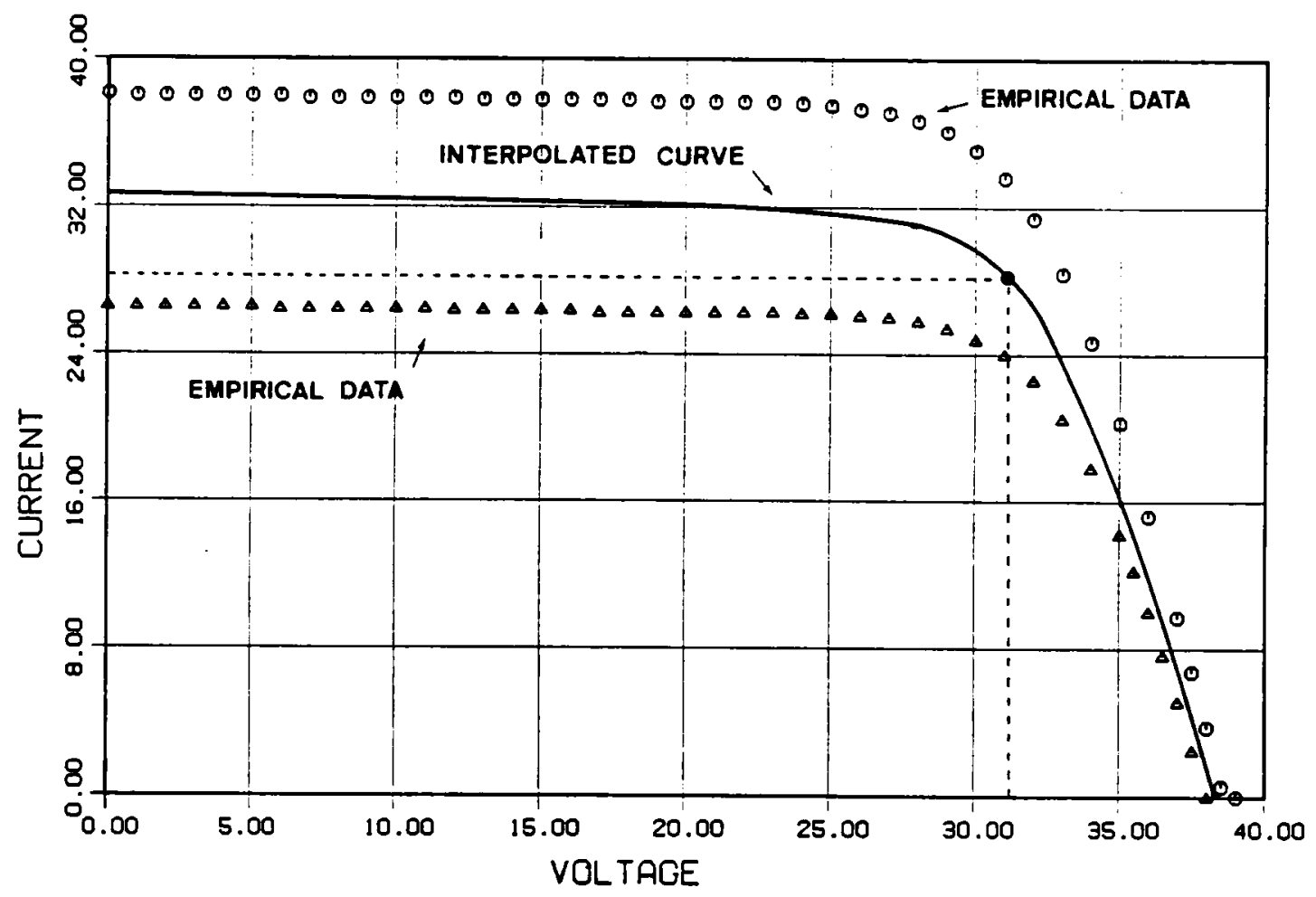

Figure 3.16 Empirical data modeling of the solar array 
Differentiating the diode equation in Eq.(3.56) and substituting Eq. (3.57), the value of $r_{d}$ at a particular solar array operating point is obtained

$$
r_{d}\left|I_{o}, V_{o}=n_{p} I_{d o} \frac{Q}{k T} \exp \left(\frac{Q V_{D}}{k T}\right)\right| V_{D}=V_{o} / n_{s}+R_{s} I_{0} / n_{p}
$$

Then the output resistance is

$$
r_{o}=R_{s}+r_{d} \| R_{s h}
$$

The characteristic of the solar array $I-V$ curve is mainly determined by $r_{d}$ and its value widely varies depending on the operating point.

\subsubsection{Solar array small signal model}

The standard small signal AC solar cell model is shown in Fig.3.17. Like the DC model, this model is simple and produces good first order results. Various techniques are given in the literatures for determination of the model parameter values. For the first order AC model, the parameters are dependent on output voltage and current, cell temperatures, cell illumination, and physical cell dimension. It is, therefore,

necessary to take these dependencies into account when model parameters are calculated. It is especially important to characterize the dynamic resistance whose value varies widely with the operating point on the solar array dc I-V characteristic. With a procedure similar to the DC model 


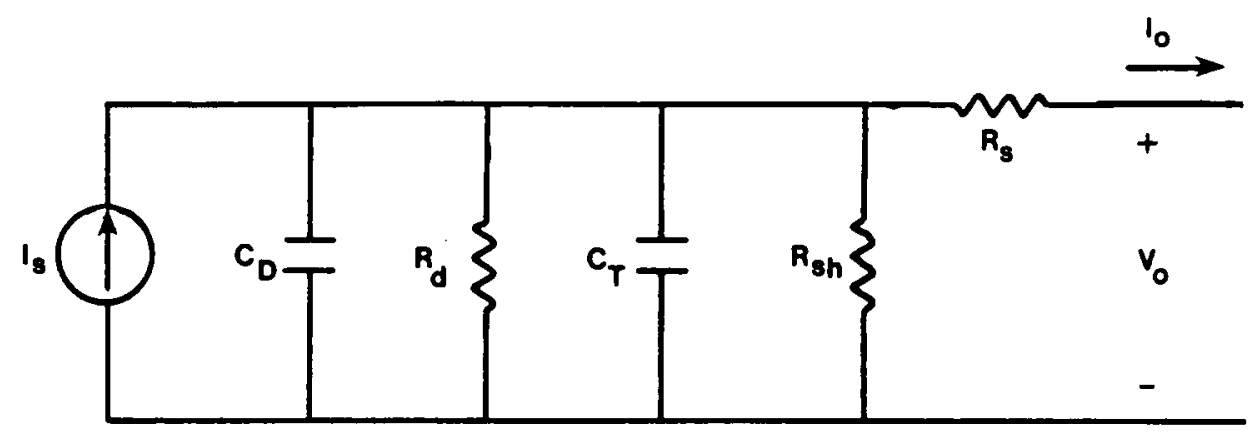

Figure 3.17 Small signal AC model of solar cell

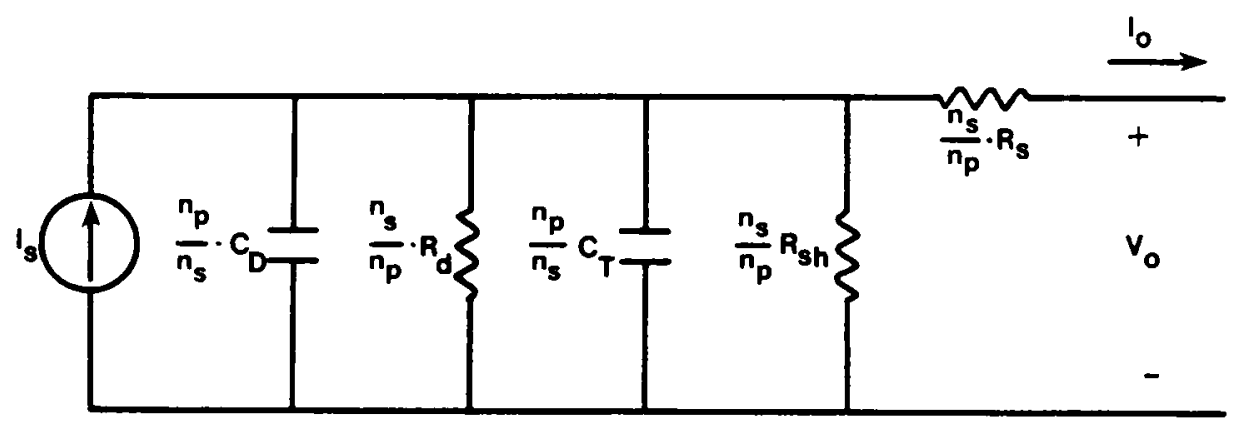

Figure 3.18 Small signal AC macro model of solar array 
generation, an equivalent small signal macro model is developed as shown in Fig.3.18. For an array model, additional circuit elements should be included to incorporate the cell interconnection. These parameter values, of course, depend on the physical construction of an array. In the present model, they are described as lumped $R, L$, and $C$ which are functions of the number of cells in series and parallel, $n_{s}$ and $n_{p}$, respectively.

From Fig. 3.18 , the output impedance is

$$
Z_{o}=R_{s}+R_{e q} \|\left(1 / s_{e q}\right)
$$

where

$$
\begin{gathered}
R_{e q}=r_{d} \| R_{s h} \\
C_{e q}=C_{T}+C_{D}
\end{gathered}
$$

As mentioned in the previous section, the characteristic of the solar array output impedance, $Z_{0}$, varies widely depending on the its dc operating condition. This nonlinear output impedance coupled with a load impedance, $Z_{L}$, may result in a system instability as the dc operating point is changed. This is particularly critical when $Z_{L}$ represents a switching regulator. The constant power characteristic of switching regulators coupled with the solar array $I-V$ curve can create multiple equilibrium points. Stability of each equilibrium point depends on the dc operating region of the solar array. The detailed stability analysis of the solar array operating point is presented in Section 5.2. 


\subsection{Shunt regulator modeling}

Shunt regulators are used to clamp the power bus voltage during periods when excess solar array power is available. There exist basically two types of shunt regulator circuits, linear type and PWM type. Modeling techniques for the PWM type have received a great deal of attention in the last two decades. A discussion of the modeling and analysis of the PWM regulator is contained in the section on Switching Regulator Modeling. The shunt regulator configurations can also be categorized by their functions, full shunt and partial shunt. In a full shunt configuration, the shunt elements are placed in parallel with the load. In a partial shunt configuration, the shunt elements only shunt part of the solar array cells. Each solar array segment is tapped by a shunt element. In this dissertation, the full shunt configuration is modeled.

Fig.3.19 illustrates the simplified block diagram of a typical shunt regulator. The basic circuit functions are as follows. A divided-down sensed bus voltage is compared to a reference voltage. The error voltage is then amplified to control the current through the shunt elements. A resistor of suitable value (depending on the maximum design current for the shunt) is used in the shunt current path to monitor the current that is flowing through the shunt elements. Before the actual shunt circuit is described, the linear analysis using the simplified circuit in Fig.3.19 will be carried out in the following section. 


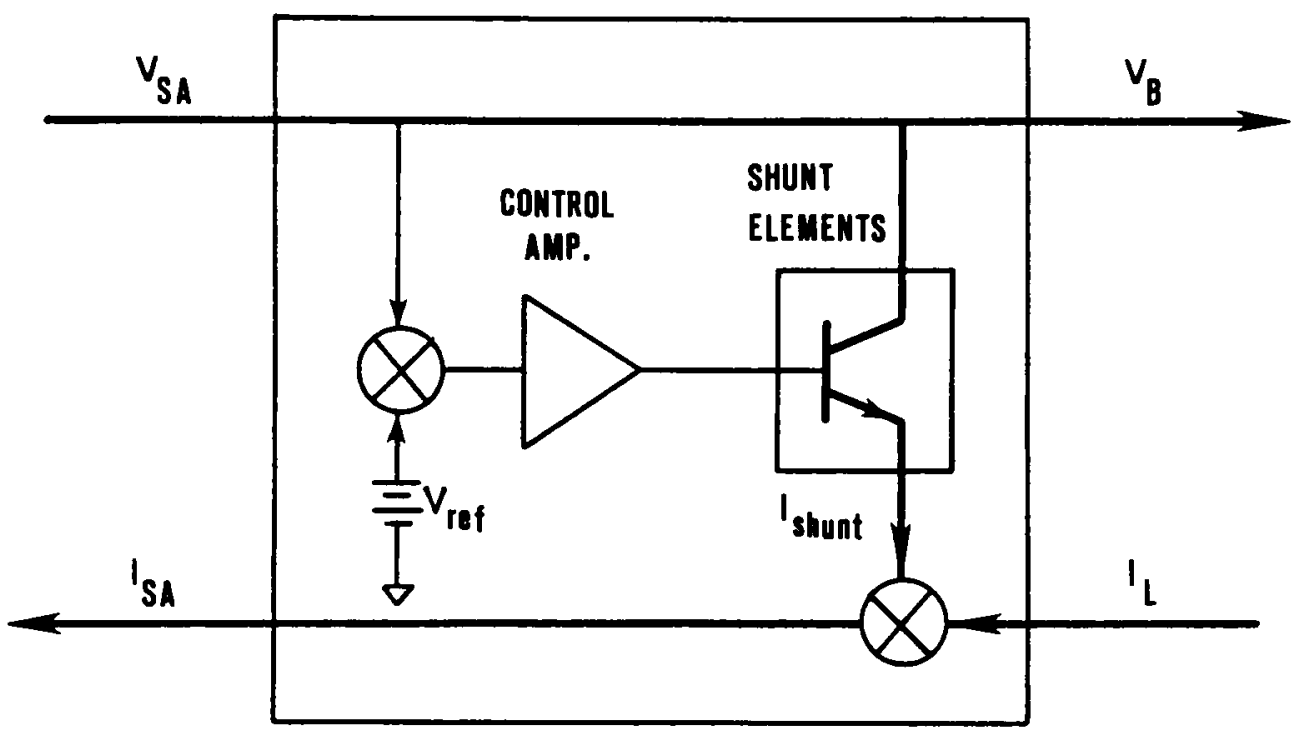

Figure 3.19 Simplified shunt regulator model

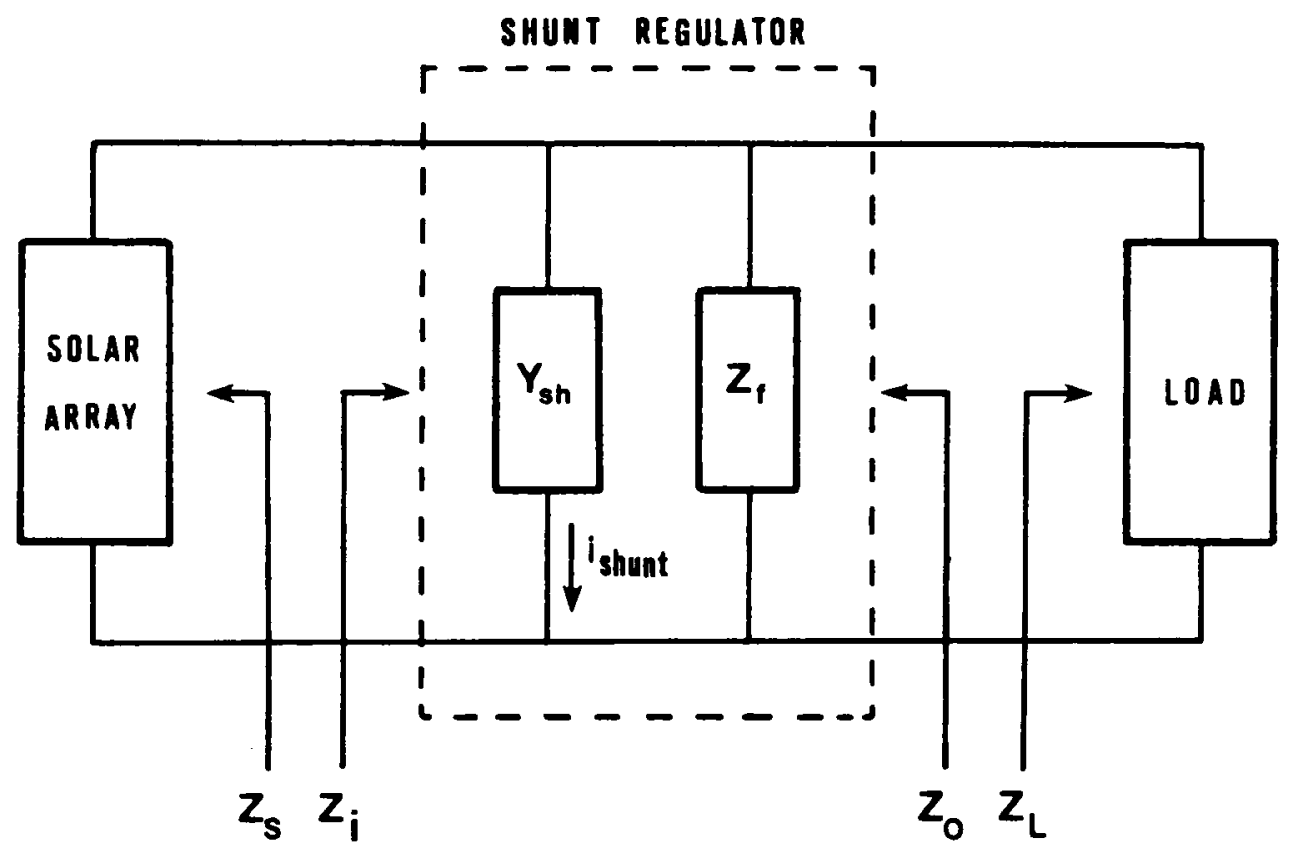

Figure 3.20 Doubly terminated shunt regulator system 


\subsubsection{Linear analysis of the shunt regulator}

Using the quantity $\mathrm{Y}_{s h}$, the transadmittance from the bus voltage to the shunt current, the impedance seen by the bus for the unterminated shunt regulator is

$$
Z_{B}=\frac{Z_{f}}{1+Z_{f} Y_{s h}}
$$

and the loop gain of the regulator is

$$
T_{G}=Z_{f} Y_{s h}
$$

The performance parameters for the doubly terminated regulator shown in Fig. 3.20 are derived as

$$
\begin{aligned}
Z_{B}= & \frac{Z_{f} \|\left(Z_{S} \| Z_{L}\right)}{1+Y_{s h}\left[Z_{f} \|\left(Z_{S} \| Z_{L}\right)\right]} \\
T & =Y_{s h}\left[Z_{f} \|\left(Z_{S} \| Z_{L}\right)\right] \\
& =T_{G} \frac{1}{1+Z_{f} /\left(Z_{S} \| Z_{L}\right)}
\end{aligned}
$$

For the load $\left(Z_{L}\right)$ terminated regulator, the input impedance is 


$$
z_{i}=\frac{z_{f} \| z_{L}}{1+Y_{s h}\left(z_{f} \| z_{L}\right)}
$$

and the loop gain of the load terminated regulator can be defined as

$$
T_{L}=Y_{s h}\left(Z_{f} \| Z_{L}\right)=T_{G} \frac{1}{1+z_{f} / Z_{L}}
$$

For the source $\left(Z_{S}\right)$ terminated regulator, the output impedance in Fig.3.20 is

$$
Z_{o}=\frac{Z_{f} \| Z_{s}}{1+Y_{s h}\left(Z_{f} \| Z_{S}\right)}
$$

and the loop gain of the source terminated regulator is

$$
T_{S}=Y_{s h}\left(Z_{f} \| Z_{S}\right)=\frac{Y_{s h} Z_{S}}{1+Z_{S} / Z_{f}}
$$

As described in [9], design and analys is of the unterminated shunt regulator performance parameters in Eqs.(3.62) and (3.63) are fairly straightforward. However, as Eqs. (3.64) through (3.69) show, stability of the regulator depends on its terminating impedances. Since the source impedance, $Z_{S}$, is the output impedance of the solar array, and the shunt regulator is supposed to be operated in the constant voltage region of the solar array, where the magnitude of $\mathrm{Z}_{\mathrm{S}}$ is small, the source 
interaction is not as critical as the load interaction and is quite predictable using Eq. (3.69).

The load characteristics of the power conditioning equipment create two areas of interaction concern in the stabilization of the shunt regulator feedback loop. First, the resonance of the input filters required the power conditioning equipment create a low impedance on the bus $\left(Z_{L}\right)$ at the resonant frequency, which may cause an oscillation or an undesirable transient response. Secondly and more importantly, because the typical load (equipment) for shunt regulator is (a) switching regulator(s), the phase of the loop gain $\mathrm{T}_{\mathrm{L}}$ in Eq. (3.67) can be shifted more than $180^{\circ}$ when its magnitude is still greater than unity due to the negative input impedance characteristic of the switching regulator. This fact established the need to have the output impedance of the shunt regulator, $Z_{B}$ and the load impedance, $Z_{L}$, well defined over the bandwidth of the loop gain.

\subsubsection{Modeling of the shunt regulator circuit}

A block diagram of an existing shunt regulator as shown in Fig.3.21 has been modeled in detail. The computer model takes into account salient dynamics and nonlinearities including op-amp feedback, op-amp saturation and darlington cut-off. All diodes, including the transistor's base-toemitter junction, have an infinite reverse impedance. The first order model is used for transistors with their parameter values (such as current gain and base-emitter junction voltage, $v_{B E}$ ) as the user's inputs. The LC low-pass filter is added, which can be considered as the input filter 

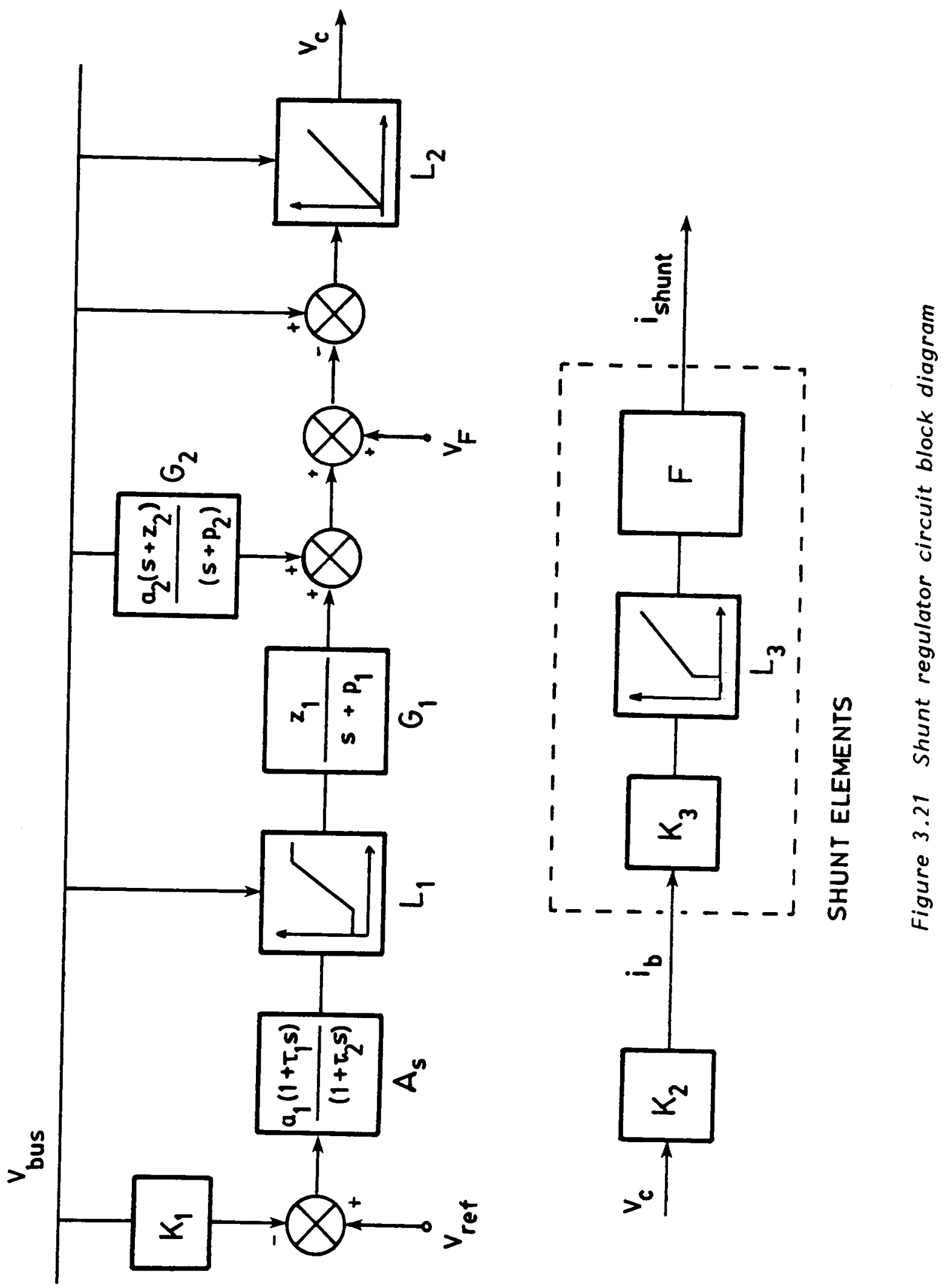
and/or the cable impedance from the solar array to the shunt regulator. It also allows preservation of the standard interconnection law providing four terminal variables.

The analytical derivation of the transadmittance $Y_{\text {sh }}$ from Fig.3.21 results

$$
Y_{s h}=\left[K_{1} A_{s} G_{1}+G_{2}\right] K_{2} K_{3} F
$$

where $K_{1}$ is the sensed voltage divider factor, $A_{s}$ the error amplifier transfer function, $G_{1}$ and $G_{2}$ the filter transfer functions, $K_{2}$ the gain from the base voltage of the darlington transistor to the base current of the shunt elements, $K_{3}$ the 12 sections of shunt elements, and $F$ the current gain of the shunt elements.

\subsection{Filter / Cable Modeling}

Spacecraft power components usually require both input and output filters to decrease andiosusceptibility and to reduce switching current ripple seen by the main bus. This is particularly important for switching converters having pulsating input or output current. Power distribution cables can also be realized in the filter model.

Fig.3.22 shows the single stage low pass filter configuration that can be used for both filter and cable models. Other multiple stage filter or n-section cable models can be constructed by cascading the model. Parameter estimation can be quite difficult for a cable model. An at- 


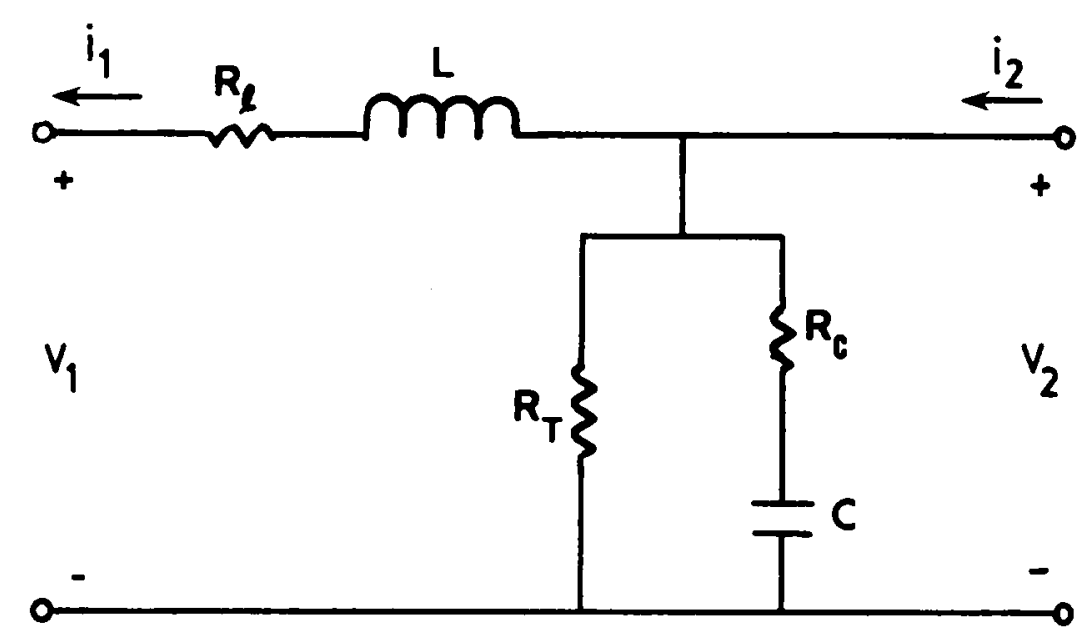

Figure 3.22 Single section of cable/filter model 
attractive modeling approach using empirical data modeling of such components is discussed in Chapter 7. From Fig.3.22, the two-port gparameters are derived as follows.

$$
G=\frac{1}{Z_{1}+z_{2}}\left[\begin{array}{cc}
-1 & z_{L} \\
z_{L} & z_{1} \cdot z_{2}
\end{array}\right]
$$

where

$$
\begin{aligned}
& \mathrm{Z}_{1}=\mathrm{R}_{\ell}+\mathrm{sL} \\
& \mathrm{Z}_{2}=\mathrm{R}_{\mathrm{T}} \|\left(\mathrm{R}_{\mathrm{c}}+1 / \mathrm{sC}\right)
\end{aligned}
$$

\subsection{Payload modeling}

Due to the diversity of the spacecraft's payloads, flexibility of the modeling technique is very important. As described in Chapter 2, the modeling techniques can use state equations, transfer functions, empirical data, or a combination of these. For experimental data modeling, the payload can be characterized by two types of data, frequency domain and time domain. The frequency domain data can be further realized with the analytical model by using the complex curve fitting technique. Details of this approach are described in Chapter 7. The time-domain data model can be obtained in tabular form when the payload is a time varying function where the dependent variable is not directly a function of system electrical operating conditions but of some external variables such as temperature, light intensity, aging, etc. 


\subsection{Conclusions}

In this chapter, components of spacecraft power systems are modeled. Each component is described with the two-port hybrid g-parameters and its interconnection law.

A component level analysis is performed by means of unterminated, singly terminated (one-port), and doubly terminated (two-port) component. The important performance parameters such as line transfer functions, input and output impedances and feedback loop gains for regulators are derived in analytical closed forms in which the terminating elements, $Z_{S}$ and $Z_{L}$ are explicitly factored out. Based on the derivations in this chapter, the system level analysis focusing on the subsystem interactions can easily be carried out as will be shown in Chapter 6 . 


\section{Chapter 4}

\section{MODELING A DIRECT ENERGY TRANSFER POWER SYSTEM WITH EASY5}

\subsection{Introduction}

Based on the component models generated in Chapter 3, a complete system can be configured by interconnecting the component models. The particular spacecraft power system under investigation is a direct energy transfer power system [10] which is described in Section 4.2. In Section 4.3, system model building with the host software EASY5 and its analysis capabilities are described.

Using the system model generated in this chapter, the capabilities of the model are demonstrated in Chapter 5 through Chapter 7, by performing various types of the system level analysis.

\subsection{Direct energy transfer spacecraft power system}

The system being modeled in this study is a direct energy transfer (DET) system whereby the primary power source, a solar array, is coupled through a main distribution bus directly to the spacecraft electrical loads. The various power-conditioning components, as shown in Fig. 4.1, are activated only as needed, thus requiring the system to process only the amount of power needed to maintain the bus at the specified voltage level. A system mode of operation is determined by the power system control unit. The control unit continuously monitors appropriate signals to activate the power conditioning equipment. The control unit provides 


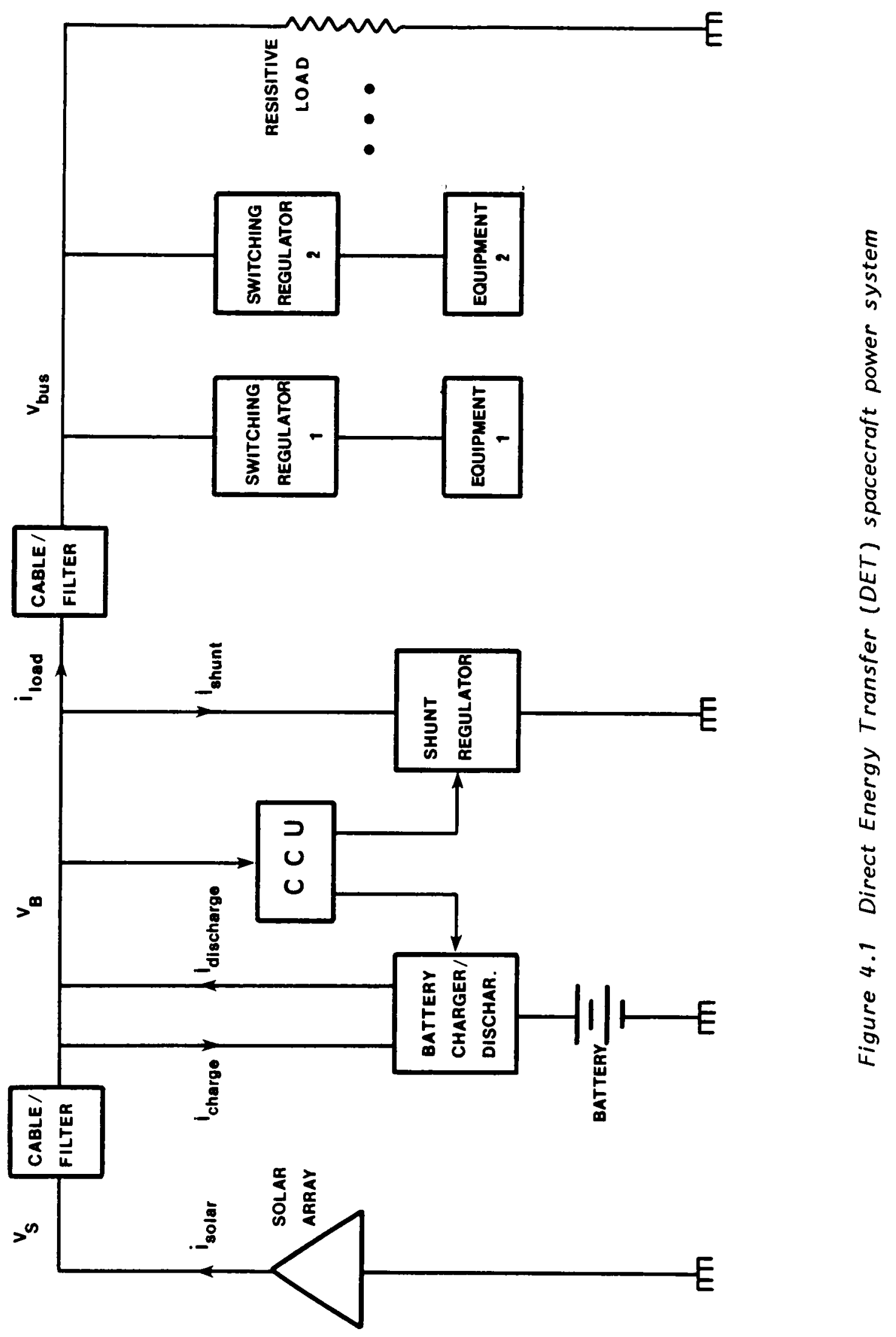


signals that control the disposition of the solar array, battery and shunt currents to maintain the bus voltage regulation. When the bus voltage exceeds the specified level, the control signal activates the shunt regulator, and the excessive solar array power is absorbed by the shunt elements. In this mode, the solar array also supplies the charging current to the battery if it is necessary. When the bus voltage becomes less than the specified level, the battery discharging electronics are activated and the load power is supplied by the solar array and the battery in shared mode. The battery discharging current is controlled by the bus voltage.

In this study, modeling of the battery and the battery charging electronic are omitted, thus, the battery is treated as an ideal voltage source. A boost type switching regulator is used for the battery discharger, which will be described in detail in Section 5.4 .

The system modes of operation of the DET system are described in Chapter 5. Since the system is configured by interconnection of various components, complex interactions among components and subsystems may occur that can cause the total system to oscillate. In Chapter 6, various analyses are performed to demonstrate that the system can be unstable due to the undesirable interactions among the individually well behaved components.

\subsection{EASY5 system model generation and analysis}

Once all the necessary components are modeled, they are stored in EASY5 macro component library. As shown in Fig. 4.2, the EASY5 consists of two 


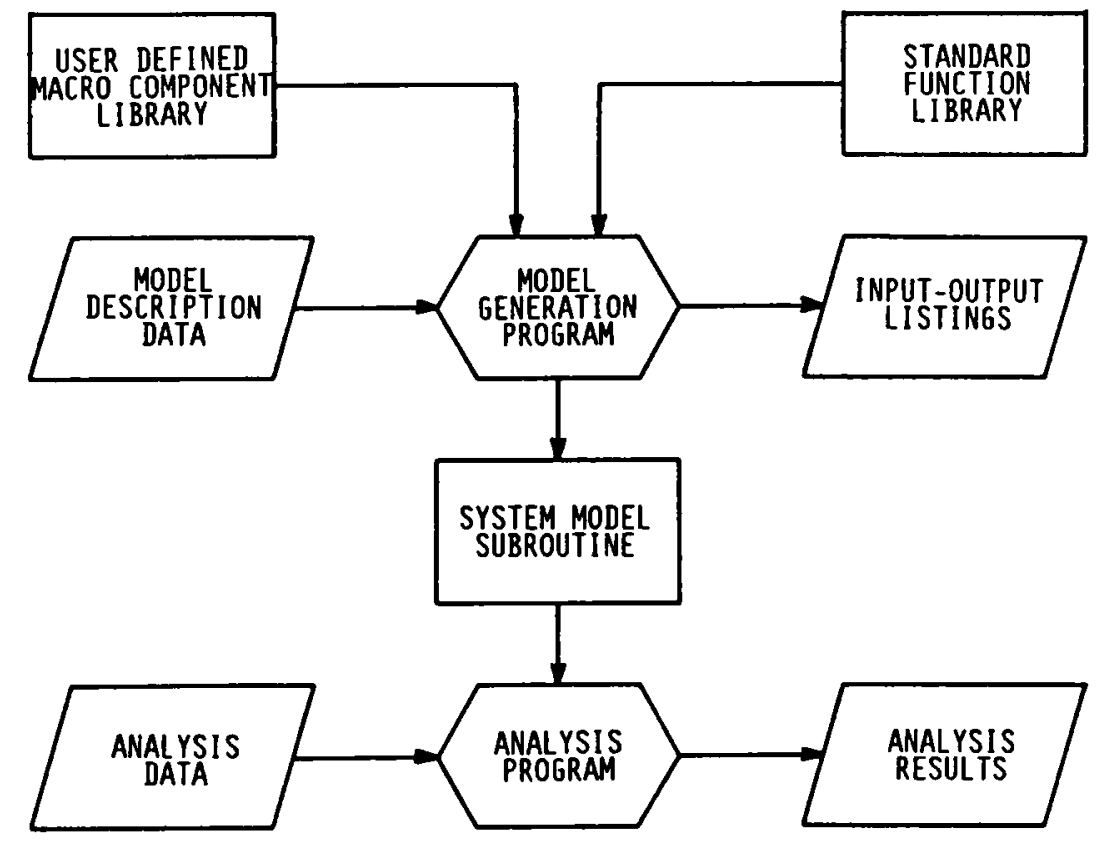

Figure 4.2 EASY5 system 
programs, and a library of predefined Standard Components. Predefined Standard component models include many of the common effects found in dynamic systems such as standard analog and discrete controllers (proportional, integral, differential, etc.) transfer functions, nonlinearities (relay, saturation, hysteresis and switches), linear state equation model, etc.

Model generation: The EASY5 Model Generation program uses a block diagram type of approach for constructing various system models. All interconnections between the component models are accomplished by the Model Generation program according to the user specified Model Description Data. This is accomplished by matching the input and output port quantities of each interconnection. Table 4.1 shows the Model Description Data of the DET system. The program also produces a complete list of input data that is required by each component to complete the model description. The scalar and vector parameters and tabular data required for the analysis are included in this list.

Analysis: Once a system model has been constructed, the Analysis program performs various dynamics analyses according to the user provided Analysis Data. The Analysis Data includes parameter values, initial conditions of states, various analysis controls and analys is commands. Among the various analysis controls in EASY5, it is important to note that the INTEGRAL control allows one to perform the analysis at various levels such as system, subsystem, or component. The analys is in the component or subsystem level is done by 'freezing' the states of the subsystem of 


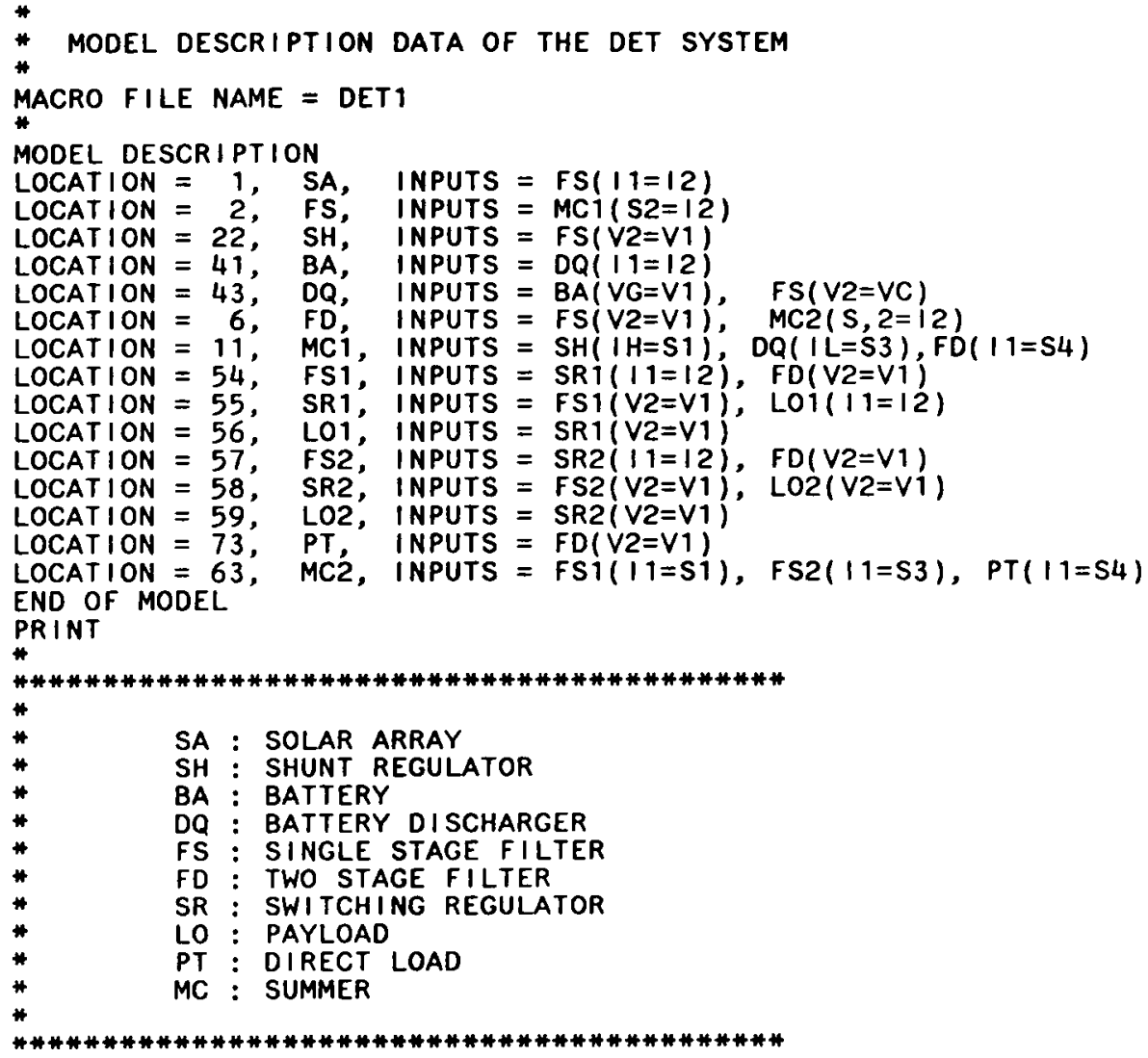

Table 4.1 EASY5 Model Description Data of the DET system 
little interest. This control feature is particularly useful when one is interested in investigating behaviors of a component or a subsystem in the presence of the entire system. A list of the analysis techniques available through the Analysis program are given as follows.

- Time history generation - nonlinear simulation

- Linear model generation with eigenvalue and eigenvector calculations

- Frequency response analysis

- Root locus calculation

- Steady state analysis

- Stability margin calculations

- Optimal controller synthesis 


\section{Chapter 5}

\section{ANALYSIS OF THE SOLAR ARRAY SYSTEM MODE OF OPERATION}

\subsection{Introduction}

Solar arrays, as the primary power source for spacecraft, generates its output electrical power relative to the illumination level. The operating point of the solar arrays is determined by the power conditioning equipment and the load characteristic. Secondary power conditioning equipment consists of a shunt regulator, a battery bank and its charging and discharging electronics. When the output power of the solar array exceeds the load demand, a portion of the excess power is used to charge the battery bank, the secondary power source, with a portion dissipated in the shunt regulator. When the load power demand is higher than the solar array output power, the battery bank supplies the excessive load power through the battery discharging electronics. The power conditioning equipment not only balances the power of the system, but also regulates the bus voltage. Since the solar array output power varies over a wide range according to the changes in the illumination level, several modes of operation exist depending on the characteristics of the solar array, the power conditioning equipment, and the load.

As described in Section 3.3.2, the solar array output characteristic is nonlinear. This nonlinear source coupled with nonlinear load characteristics may result in multiple equilibrium points under a given operating condition. The actual equilibrium state that the system resides 
on is determined by the stability nature of the equilibrium points and the past history. Thus, it is necessary to analyze the stability of the equilibrium points and to understand various modes of operation of the system.

In Section 5.2, stability of the equilibrium points of a solar array system with a nonlinear load characteristic is analyzed. The behavior of different solar array operating points are plotted as trajectories on the state plane. Stability and transient responses of the system operating near the solar array maximum power point is considered in Section 5.3.

In Section 5.4, the operation during battery discharge is analyzed including the transition mode from the shunt regulator active mode. In the direct energy transfer (DET) system, the battery discharger directly regulates the main bus voltage. Since the solar array output power varies over a wide range, the battery discharge converter operates from no-load to full load. Under light load, the converter operates in the discontinuous conduction mode where the energy storage inductor current is reduced to zero during a portion of a switching cycle. The DC and dynamic behaviors of a switching converter in the discontinuous conduction mode are quite different than that of the continuous conduction mode. Modeling of the discontinuous conduction mode employing the state space averaging is described in Section 5.4. Since the battery discharger also functions as the bus voltage regulator, the feedback compensator design for the converter in both modes of operation are also discussed.

In Section 5.5, various modes of operation of the DET system are described. Conclusions are presented in Section 5.5. 


\subsection{Large signal behavior of the solar array operating point}

Fig.5.1 illustrates a solar array power system, in which the static load line represents a typical buck type regulator load line characteristic. The voltage $V_{R}$ in the figure is the minimum solar array output voltage above which the converter is able to regulate its output voltage. When the converter is regulating, the solar array sees a constant power. Note that the converter exhibits a negative resistance characteristic in this mode. Below $V_{R}$, the converter behaves as a resistive load to the solar array.

As shown in Fig.5.1, there may exist three equilibrium points in the system. The large signal behavior of a solar array operating point depends on the stability nature of each equilibrium point. The qualitative large signal analysis can be carried out by considering a second order system shown in Fig.5.2. The solar array output and the load characteristics are replaced by the nonlinear functions and the LC low-pass filter in the model represents actual physical elements in the system such as an input filter of the switching regulator or the cable impedance between the solar array and the load.

Let us first consider the stability nature of each equilibrium point. Defining the states $x=\left[\begin{array}{ll}i_{L} & v_{C}\end{array}\right]^{T}$, the system state equations are

$$
\begin{aligned}
& \frac{d i_{L}}{d t}=\frac{1}{L}\left(v_{S}-v_{C}\right)=\frac{1}{L}\left(-f\left(i_{L}\right)-v_{C}\right) \\
& \frac{d v_{C}}{d t}=\frac{1}{C}\left(i_{L}-i_{R}\right)=\frac{1}{C}\left(-f\left(v_{C}\right)-i_{L}\right)
\end{aligned}
$$




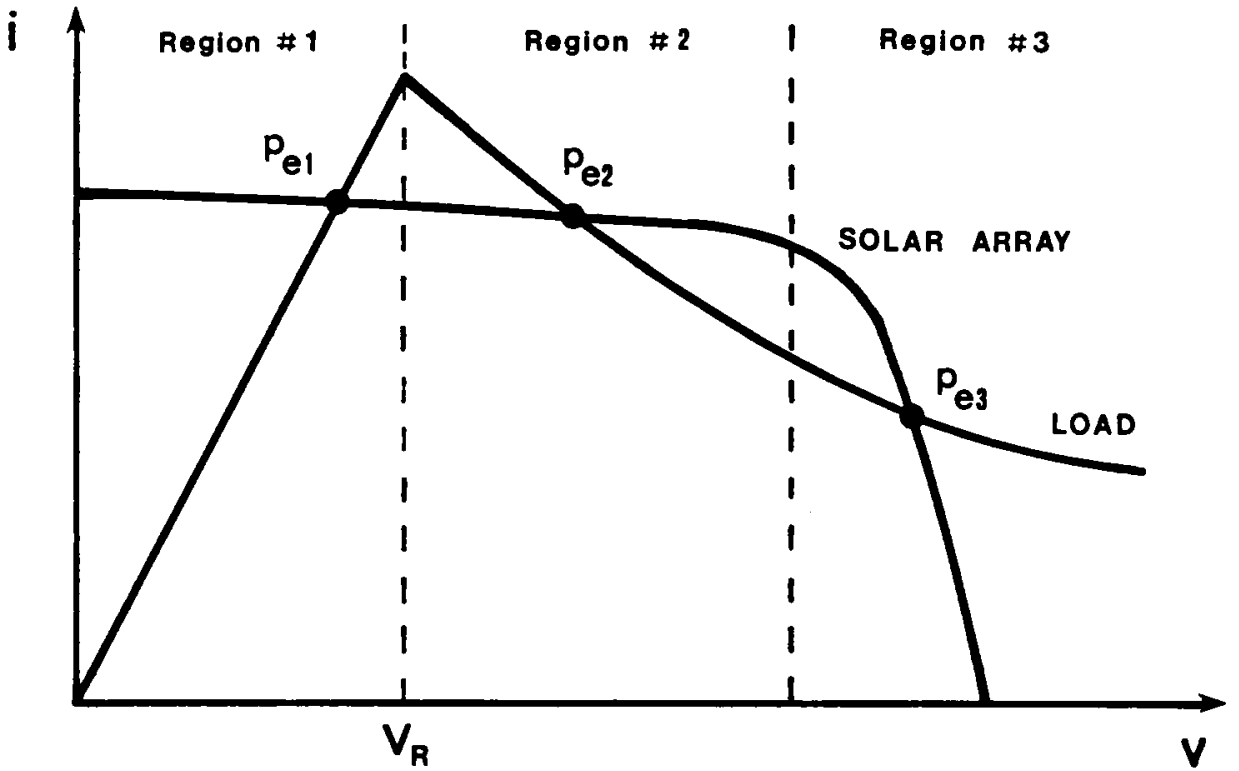

Figure 5.1 Representation of a solar array power system with a switching regulator load line

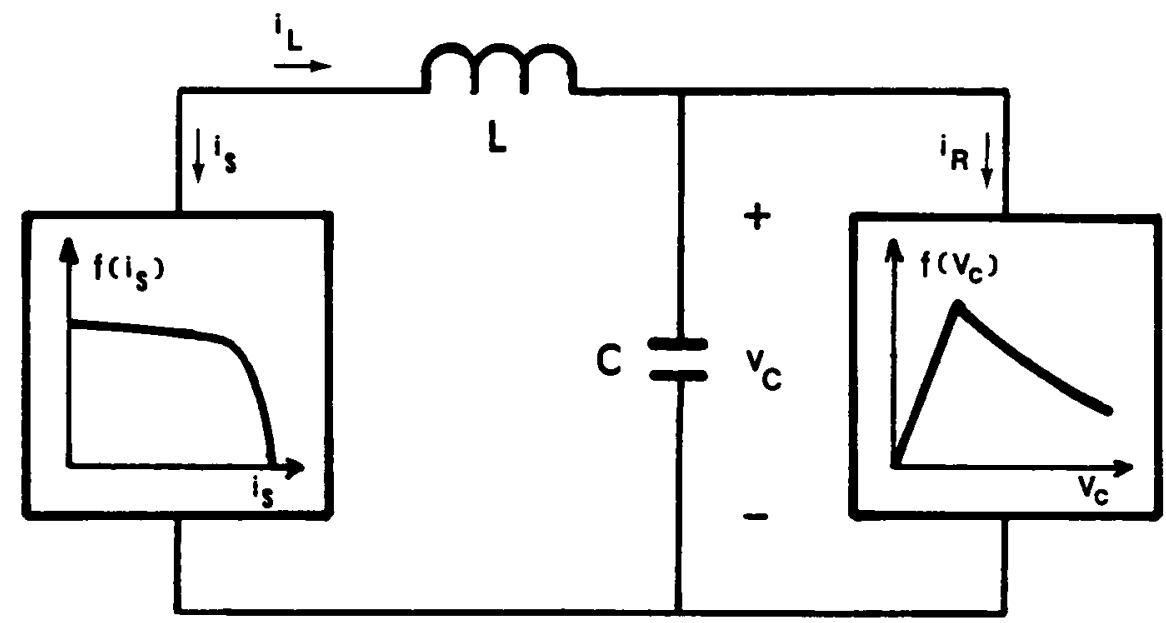

Figure 5.2 Equivalent circuit model of the system in Fig.5.1 including $L C$ filter 
where $v_{S}$ and $i_{R}$ are defined in Fig.5.2.

From Eqs. (5.1) and (5.2), the equilibrium points in Fig.5.2 are

$$
\begin{aligned}
& V_{C}=-f\left(I_{L}\right) \\
& I_{L}=f\left(V_{C}\right)
\end{aligned}
$$

The behavior of the trajectories in the neighborhood of an equilibrium point can be found by linearizing Eqs.(5.1) and (5.2) about an equilibrium point. The nature of these equilibrium points is analyzed by introducing small perturbations about the equilibrium points.

$$
\begin{aligned}
& i_{L}=I_{L}+i_{\ell} \\
& v_{C}=v_{C}+v_{C}
\end{aligned}
$$

where $I_{L}$ and $V_{C}$ represent the steady state quantities, and $i_{\ell}$ and $v_{C}$ are the small signal perturbations.

Substituting Eqs.(5.5) and (5.6) into (5.1) and (5.2) results in

$$
\begin{aligned}
& \frac{d i_{L}}{d t}=\frac{1}{L}\left[-f\left(I_{L}+i_{\ell}\right)-\left(v_{C}+v_{C}\right)\right] \\
& \frac{d v_{C}}{d t}=\frac{1}{C}\left[-f\left(V_{C}+v_{c}\right)-\left(I_{L}+i_{\ell}\right)\right]
\end{aligned}
$$


Expanding Eqs.(5.7) and (5.8) in a Taylor series about the equilibrium points, the equations of the first order approximation are

$$
f\left(I_{L}+i_{\ell}\right)=f\left(I_{L}\right)+r_{S} i_{\ell}
$$

where

$$
r_{S}=\left.\frac{d f\left(i_{L}\right)}{d i_{L}}\right|_{I_{L}}
$$

and similarly,

$$
f\left(V_{C}+v_{c}\right)=f\left(V_{C}\right)+\frac{1}{r_{L}} v_{c}
$$

where

$$
\frac{1}{r_{L}}=\left.\frac{\operatorname{df}\left(v_{C}\right)}{d v_{C}}\right|_{v_{C}}
$$

where $r_{S}$ and $r_{L}$ are the incremental resistances (tangential slope) of the solar array output curve and the load line, respectively, at the equilibrium points.

Employing Eqs.(5.3) through (5.10), Eqs.(5.7) and (5.8) can be simplified as

$$
\frac{d i_{\ell}}{d v_{c}}=\frac{(1 / L)\left(-r_{S} i_{\ell}-v_{c}\right)}{(1 / C)\left(i_{\ell}-\left(1 / r_{L}\right) v_{c}\right)}
$$


Note that the value of ${ }^{r_{S}}$ is the same as the output resistance at a point on the solar array $I-V$ curve as derived in Eq.(3.60). The system eigenvalues are expressed as

$$
\lambda_{1}, \lambda_{2}=\frac{1}{2} \cdot\left[-\left(\frac{{ }^{r_{S}}}{L}+\frac{1}{r_{L} C}\right)+\left[\left(\frac{{ }^{r} S}{L}+\frac{1}{r_{L} C}\right)^{2}-\frac{4}{L C}\left(1+\frac{{ }^{r} S}{r_{L}}\right)\right]^{\frac{1}{2}}\right]
$$

From Eq.(5.12), the stability of each equilibrium point can be determined for given parameter values. For the load line shown in Fig.5.1, the following results are observed.

In Region 非: $\mathrm{r}_{\mathrm{L}}>0$

The equilibrium point, $P_{e 1}$ is stable and is either "node" (real) or "focus" (complex).

In Region 非: $\quad r_{L}<0,\left|r_{S}\right|<\left|r_{L}\right|$

$\lambda_{1}$ and $\lambda_{2}$ are both real but of opposite sign, and Point $\mathrm{P}_{\mathrm{e} 2}$ is a "saddle" and is unstable.

In Region 非: $r_{L}<0,\left|r_{S}\right|<\left|r_{L}\right|$

The stability of Point $\mathrm{P}_{\mathrm{e} 3}$ depends on the parameter values.

Since the normal mode of operation should be in Region $\# 3$, the values of $\mathrm{L}$ and $\mathrm{C}$ in Fig.5.2 must be chosen to provide the stable equilibrium points in this region and to allow further analysis. However, it must be noted 
that the equilibrium points in Region $\#_{1}$ are always stable and those in region $\# 2$ are always unstable (saddle points) regardless of the parameter values. The value of ${ }^{r} s$ for each equilibrium point can be obtained using Eq. (3.49). The value of $r_{L}$ in the constant power region is

$$
r_{L}=\left.\frac{\partial v_{c}}{\partial i_{r}}\right|_{V_{C}, I_{R}}=-\left.\frac{P_{C}}{i_{r}{ }^{2}}\right|_{i_{r}=I_{R}}
$$

Approximate values of $r_{S}$ and $r_{L}$ can be found simply by drawing tangential slopes to the solar array $I-V$ curve and load line, respectively, for each equilibrium point.

To construct a state plane trajectory the isoclines for each equilibrium point are derived using Eq. (5.11).

$$
\begin{aligned}
& \frac{d i_{\ell}}{d v_{c}}=0 \rightarrow i_{\ell}=-\frac{1}{r_{S}} v_{c} \\
& \frac{d i_{\ell}}{d v_{c}} \rightarrow \infty \rightarrow i_{\ell}=\frac{1}{r_{L}} v_{c}
\end{aligned}
$$

The results in Eqs.(5.14) and (5.15) are the tangential slopes to the source and load curve at each equilibrium point, thus the trajectories cross the solar array curve horizontally and the load line vertically. Near the equilibrium points which have real eigenvalues, the trajectories 
follow the slope of the eigenvectors of the system, $\gamma$.

$$
\gamma_{1}, \gamma_{2}=\frac{L}{2} \cdot\left[-\left(\frac{r_{S}}{L}-\frac{1}{r_{L} C}\right)+\left[\left(\frac{r_{S}}{L}-\frac{1}{r_{L} C}\right)^{2}-\frac{4}{L C}\right]^{\frac{1}{2}}\right]
$$

Using the above results, the state plane trajectories can be sketched. Fig.5.3 illustrates the trajectories for the case where the equilibrium point $\mathrm{P}_{\mathrm{e} 3}$ is a stable node. As shown in the figure, the trajectories are separated by the separatrix that has the slope $\gamma_{2}$ at $\mathrm{P}_{\mathrm{e} 2}$. The two starting points $P_{1}$ and $P_{2}$ in Fig 5.3 approach two entirely different steady states, even though the initial states differ by only a small amount .

For the solar array model used in this study the following cases are set up for the computer simulations. As mentioned above, if we assume that point $\mathrm{P}_{\mathrm{e} 3}$ in Region $\# 3$ is stable then there can be four possible cases:

$$
\begin{array}{rll}
\text { i) } & P_{e 1} \text { - node, } & P_{e 3} \text { - node } \\
\text { ii) } & P_{e 1} \text { - node, } & P_{e 3} \text { - focus } \\
\text { iii) } P_{e 1} \text { - focus, } & P_{e 3} \text { - node } \\
\text { iv) } P_{e 1} \text { - focus, } & P_{e 3} \text { - focus }
\end{array}
$$

However, investigation of the cases $i$ ) and $i i)$ are sufficient to understand the behaviors of the system. The results from simulating these 


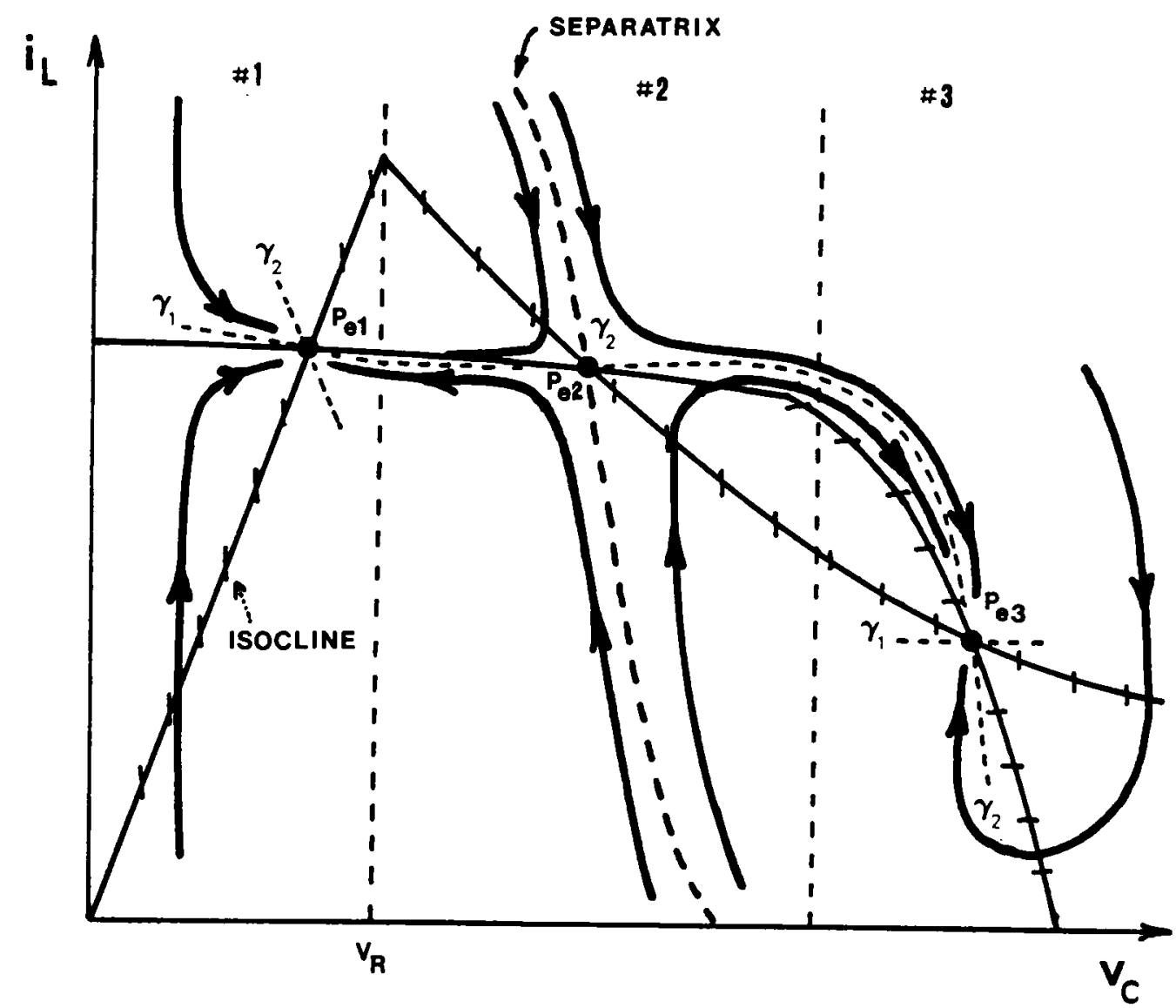

Figure 5.3 State-plane trajectories of the system in Fig.5.2 
cases are shown in Fig.5.4a and 5.4b given the following system operating conditions.

The solar array short circuit current, Iss, is $45.41 \mathrm{~A}$, the open circuit voltage, $\mathrm{V}_{\mathrm{oc}}$, is $32.0 \mathrm{~V}$, and the maximum power is 1050 watts. The constant power load line is set to 850 watts, and the boundary of Region $\equiv_{1} 1$ and 非, $V_{R}$, is $15 \mathrm{~V}$. The values of $r_{S}$ and $r_{L}$ at the equilibrium points in each region are listed in the following table.

$\begin{array}{cccc} & P_{\text {e1 }} & P_{\text {e2 }} & P_{\text {e3 }} \\ I_{L} & 45.38 & 45.02 & 30.34 \\ V_{C} & 12.03 & 17.31 & 28.02 \\ r_{S} & 64.92 & 37.3 & 0.20 \\ r_{L} & 0.025 & -0.56 & -1.47\end{array}$

The cases i) ( $\mathrm{P}_{\mathrm{e} 3}$ node) and ii) ( $\mathrm{P}_{\mathrm{e} 3}$ focus) are shown in Fig.5.4a and 5.4b. The eigenvalues and the slope of the eigenvectors for the particular values of $L$ and $C$ are 1 isted in the figure. In Fig.5.4, the separatrix is clearly illustrated. It is obtained by adjusting the initial condition that is located near the line which has been estimated from information about $\gamma_{2}$ at $\mathrm{P}_{\mathrm{e} 2}$. The trajectories from the initial points in the left-hand side of the separatrix $\left(P_{1}\right.$ through $\left.P_{6}\right)$ converge to $P_{e 1}$, and those in the right-hand side of the separatrix $\left(P_{7}\right.$ through $\left.P_{12}\right)$ to $\mathrm{P}_{\text {e3 }}{ }^{\circ}$

Since in Region $\#_{1} 1$ the system bus voltage is not regulated, the desirable mode of operation should be in Region 非. This implies that there 


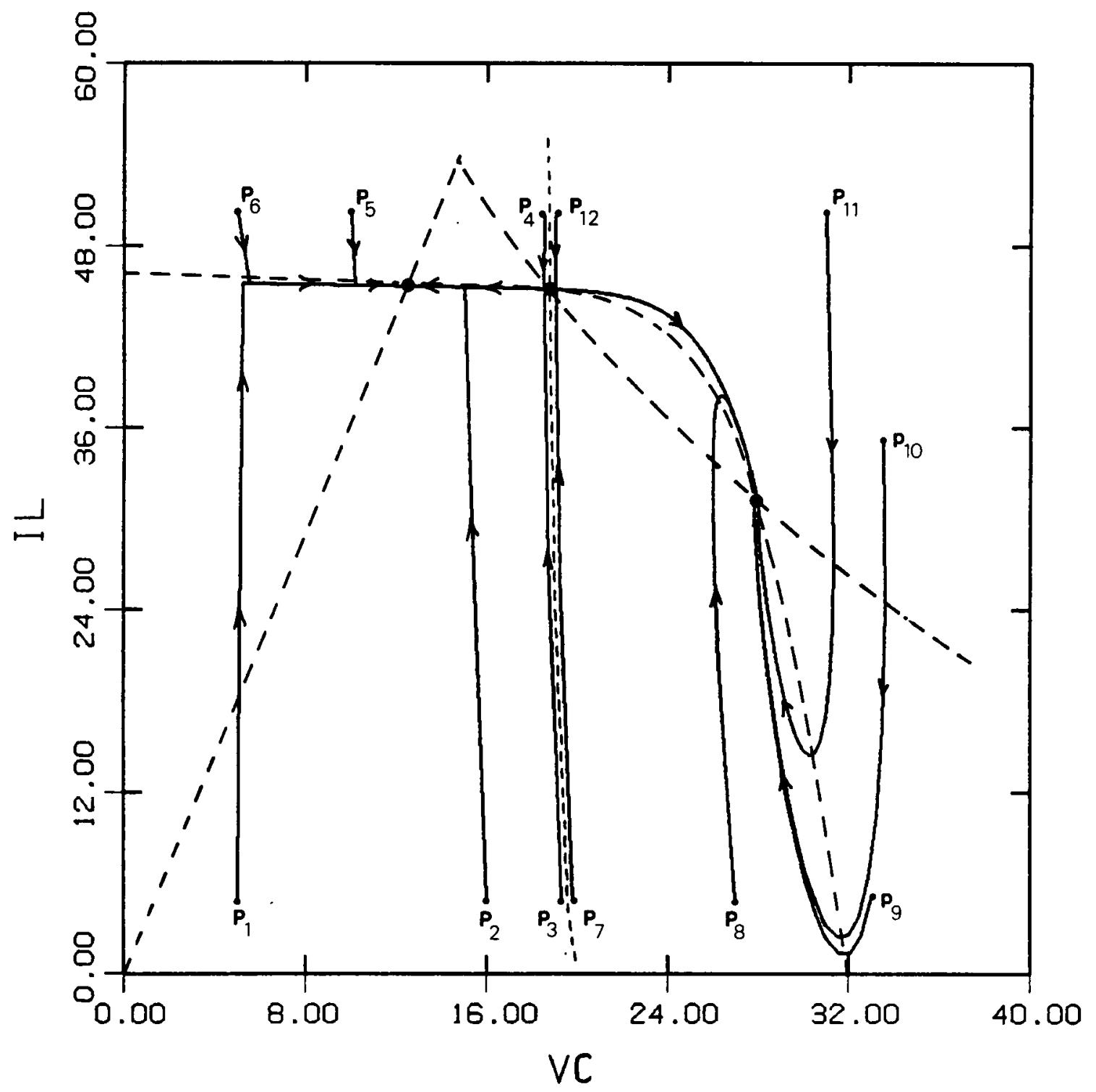

Figure $5.4 a$ Simulation of state-plane trajectories for the case where the equilibrium point $P_{e 3}$ is 'stable node' $(L=10 \mu \mathrm{H}, \quad C=500 \mu \mathrm{F})$ 


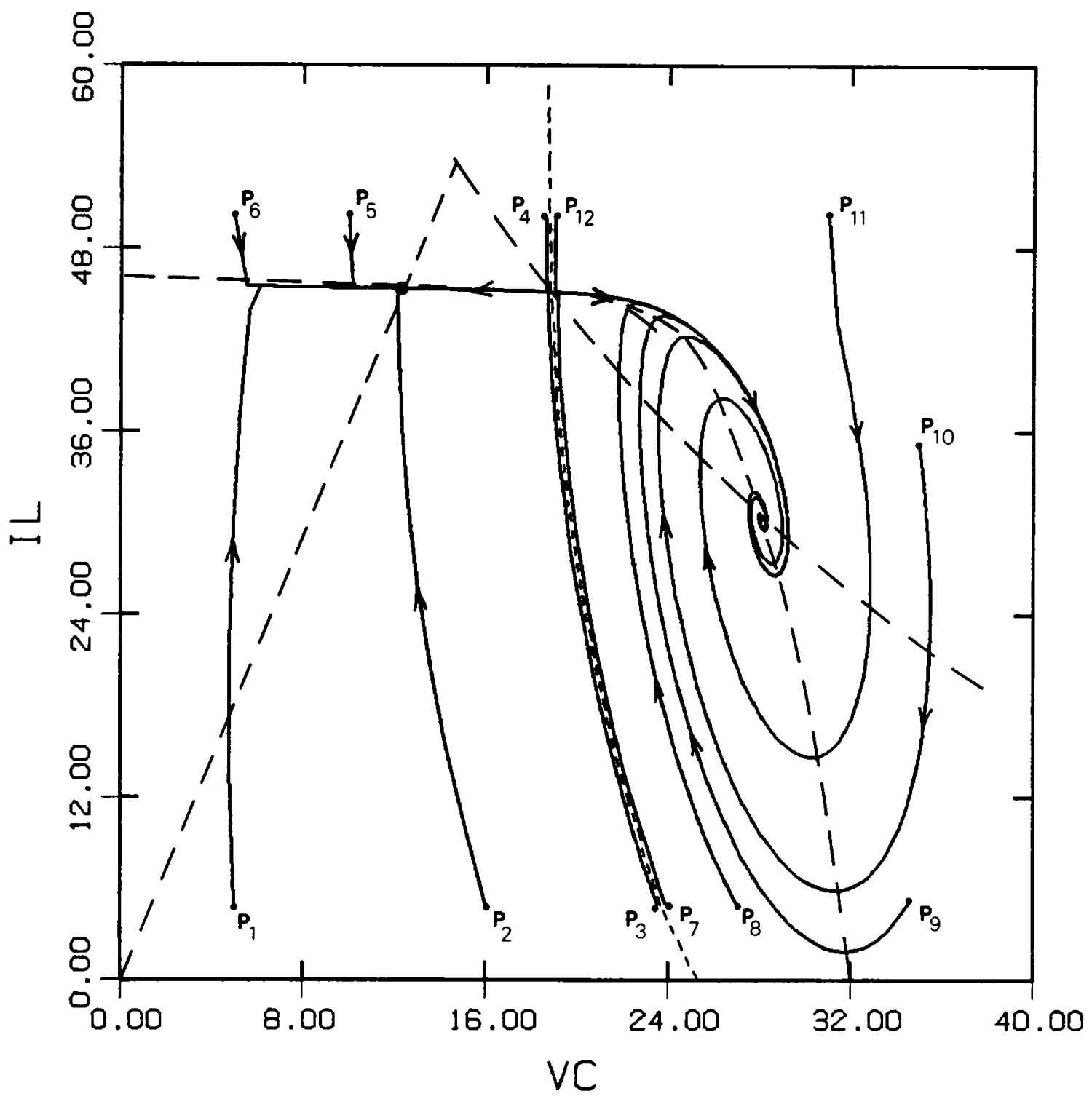

Figure $5.4 b$ Simulation of state-plane trajectories for the case where the equilibrium point $P_{e 3}$ is 'stable focus'

$$
(L=30 \mu \mathrm{H}, \quad C=500 \mu F)
$$


needs to limiting mechanism that prevents the operating point from settling into Region $k_{1} 1$ or a triggering method to move it to Region $\# 3$ even if the operating point starts in Region $\# 1$. The limiting mechanism, by means of battery will be described in the following section. A triggering method is illustrated as follows.

Suppose that the operating point is at rest in Region 非. One way to move this operating point into Region $\# 3$ is to decrease the load power below the solar array curve so that the only one desired equilibrium point exists. For the load line shown in Fig. 5.5, the equilibrium points associated with Region $\# 1$ and $\#_{2} 2$ have moved outside these regions, so that they no longer exist. However, the behavior of the state trajectories can be still seen by defining virtual equilibrium points, $\mathrm{P}_{\mathrm{e} 1}$ ' and $\mathrm{P}_{\mathrm{e} 2}$ ', for which the slopes of the eigenvectors are also sketched.

Fig.5.6 shows the simulation result using the previous system for the case i). In Fig.5.6a, the trajectory starts from an arbitrary initial point, $P_{1}$ and moves to $P_{2}$ for a load power of 800 watts (Curve $\$ 1$ ). As the load power continuously decreases, the operating point moves along the solar array curve until it reaches Curve $\$ 2$. Further decrease in the load power beyond this point moves the operating point from $\mathrm{P}_{3}$ to $\mathrm{P}_{4}$, then to $\mathrm{P}_{5}$ for Curve $\$ 3$. Fig.5.6b shows the time history of $\mathrm{v}_{c}$ corresponding the trajectory from $P_{1}$ to $P_{5}$. The above stability analysis for the system in Fig.5.2 can be extended to following cases.

System in the battery discharging mode: Fig.5.7 illustrates the system in the battery discharging mode of operation. As it will be 


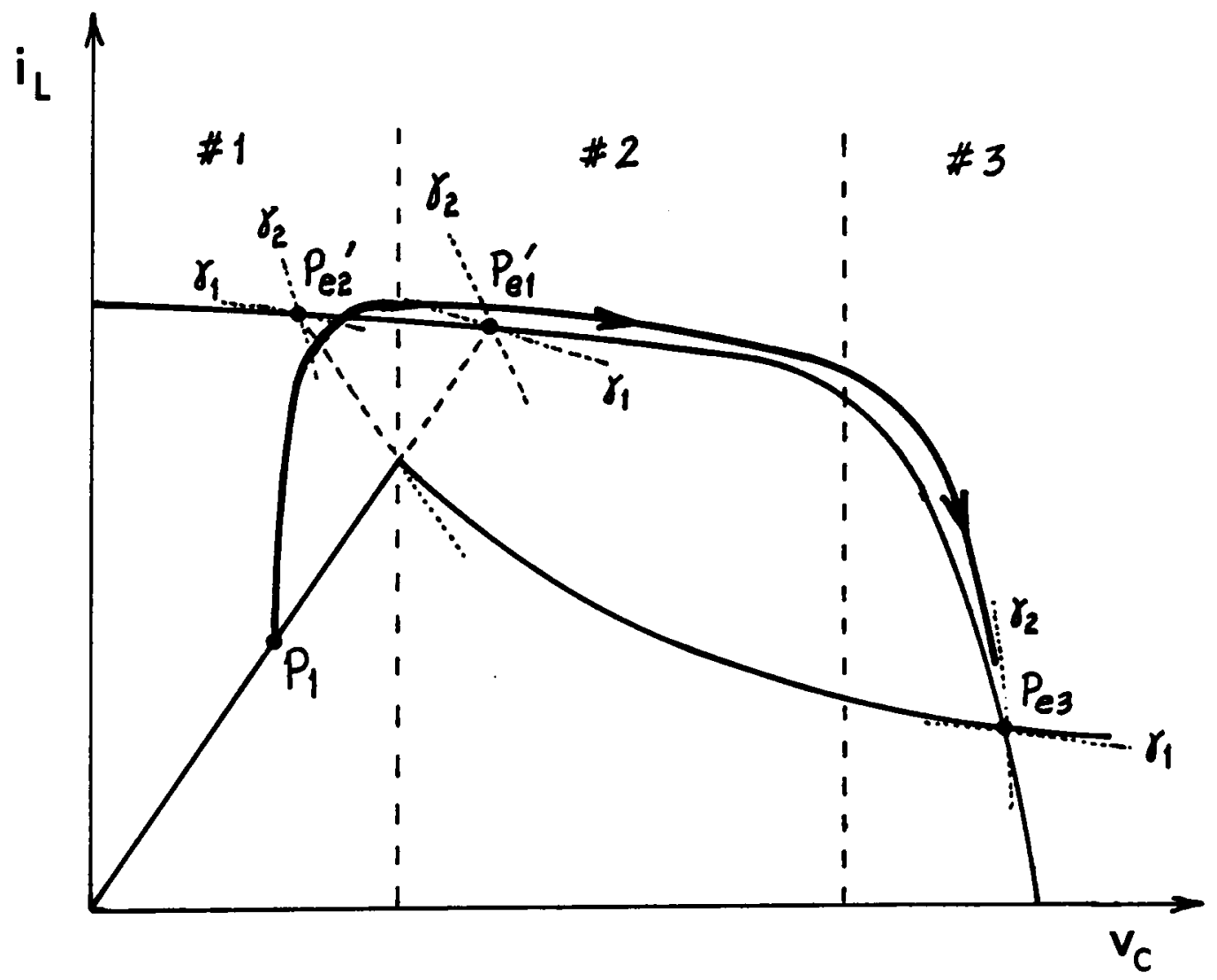

Figure 5.5 A trajectory from the initial operating point in Region \#1 to Region \#2 

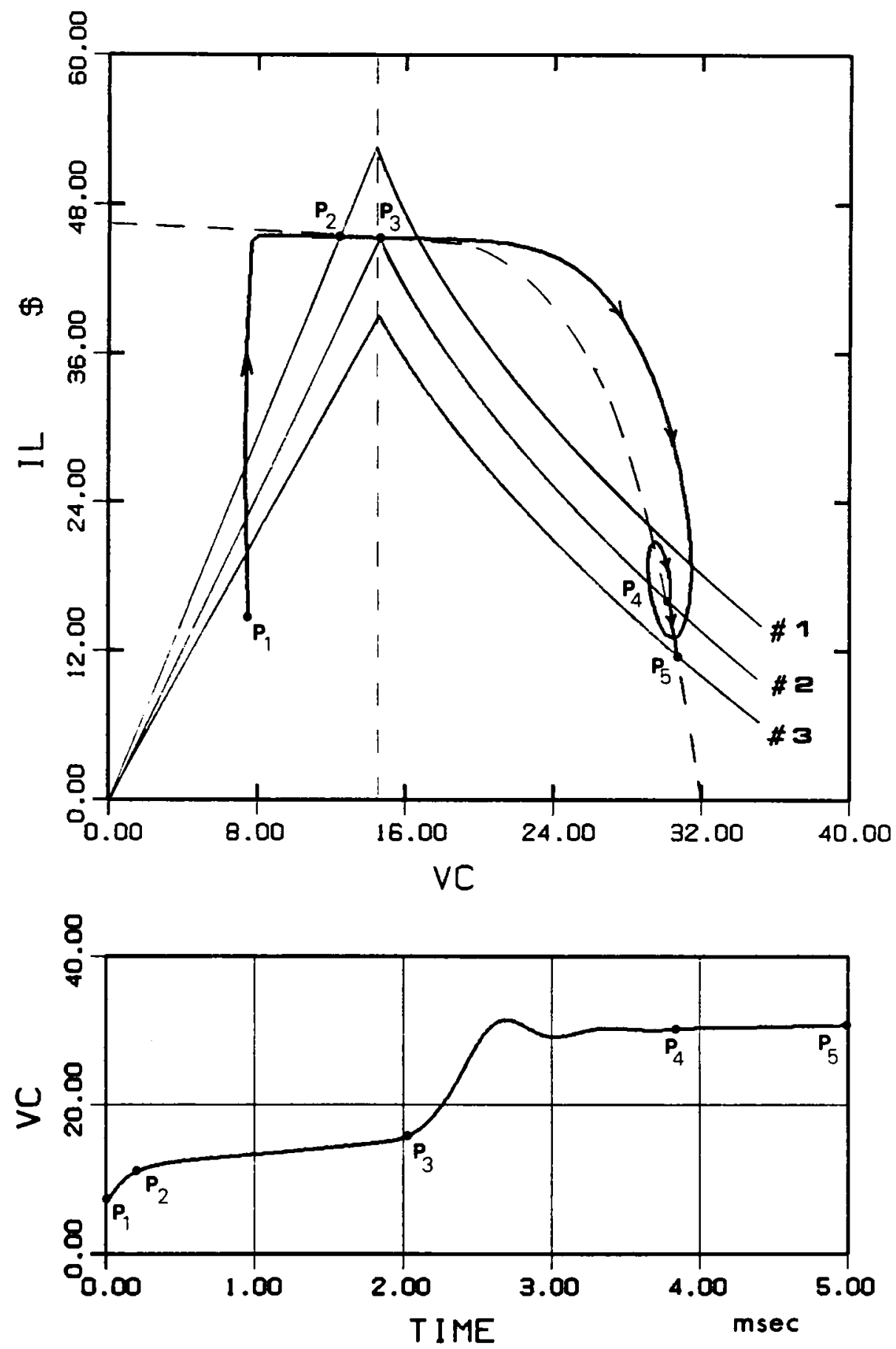

Figure 5.6 Simulation of the triggering method from the undesired stable region to the desired region (a) state-plane trajectory

(b) time history of the bus voltage 
described in detail in the following section, the battery discharging current can be expressed as a function of the bus voltage with an output admittance, $1 / r_{B}$. The state equations are then,

$$
\begin{aligned}
& \frac{d i_{L}}{d t}=\frac{1}{L}\left(-f\left(i_{L}\right)-v_{C}\right) \\
& \frac{d v_{C}}{d t}=\frac{1}{c}\left(i_{L}-\frac{1}{r_{B}} v_{C}-f\left(v_{C}\right)\right)
\end{aligned}
$$

Following the same procedures as in the previous derivations, the state equations near the equilibrium point are

$$
\frac{d i_{\ell}}{d v_{c}}=\frac{(1 / L)\left(-r_{s} i_{\ell}-v_{c}\right)}{(1 / C)\left[i_{\ell}-\left[\left(1 / r_{L}\right)+\left(1 / r_{B}\right)\right] v_{c}\right]}
$$

Then the eigenvalues are obtained as

$$
\lambda_{1}, \lambda_{2}=\frac{1}{2}\left[-\left(\frac{r_{S}}{L}+\frac{1}{r_{E} C}\right)+\left[\left(\frac{r_{S}}{L}+\frac{1}{r_{E} C}\right)^{2}-\frac{4}{L C}\left(1+\frac{r_{S}}{r_{E}}\right)\right]^{\frac{1}{2}}\right]
$$

where

$$
r_{E}=r_{L} \| r_{B}
$$

Comparing Eqs.(5.12) and (5.20), the additional term, $1 / r_{B}$, stabilizes the system, and this can be explained as the system is operating on the 


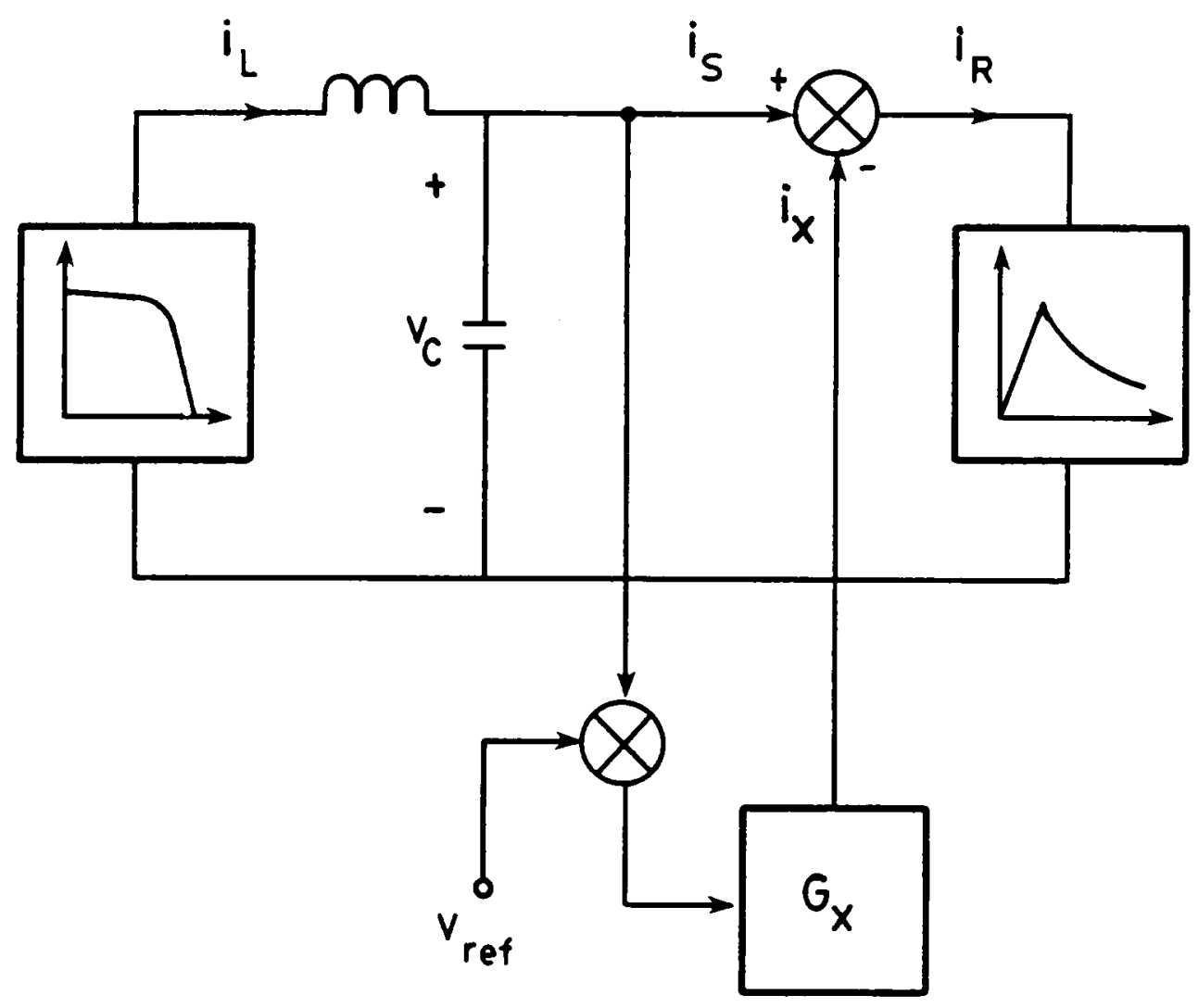

Figure 5.7 Simplified block diagram of the system in Fig.5.2 with the shunt regulator or the battery discharger

$$
G_{x}= \begin{cases}Y_{s h}: & \text { for shunt mode } \\ 1 / r_{B}: & \text { for battery discharge mode }\end{cases}
$$


stiff voltage source line.

System in the shunt mode: Fig.5.7 also can be used to illustrate the system in the shunt mode of operation. In this mode of operation, Eqs. (5.17) and (5.18) are valid if the $1 / r_{B}$ term is replaced by the shunt transadmittance, $\mathrm{Y}_{\mathrm{sh}}$. Fig.5.8 shows the state plane trajectories and the time history of the states, with and without the shunt regulator. It clearly shows the effect of the additional term described above such that the system without the shunt regulator is quite oscillatory while that with the shunt regulator has faster responses and the system appears to have real valued eigenvalues.

\subsection{Analysis of the system operating near the solar array maximum power point.}

To utilize the maximum available power from the solar array, several approaches were proposed in [11] and [12] to place the operating point as close to the solar array maximum power point as possible. However, near the solar array maximum power point, the dynamic resistance of the solar array is very sensitive to changes in the operating condition and, consequently, may result in system open loop instability.

To derive the solar array maximum power point, one can differentiate the constant power load line, $P_{a}=V I$, and set it to zero.

$$
\frac{\partial P}{\partial V}=V \frac{\partial I}{\partial V}+I=0
$$



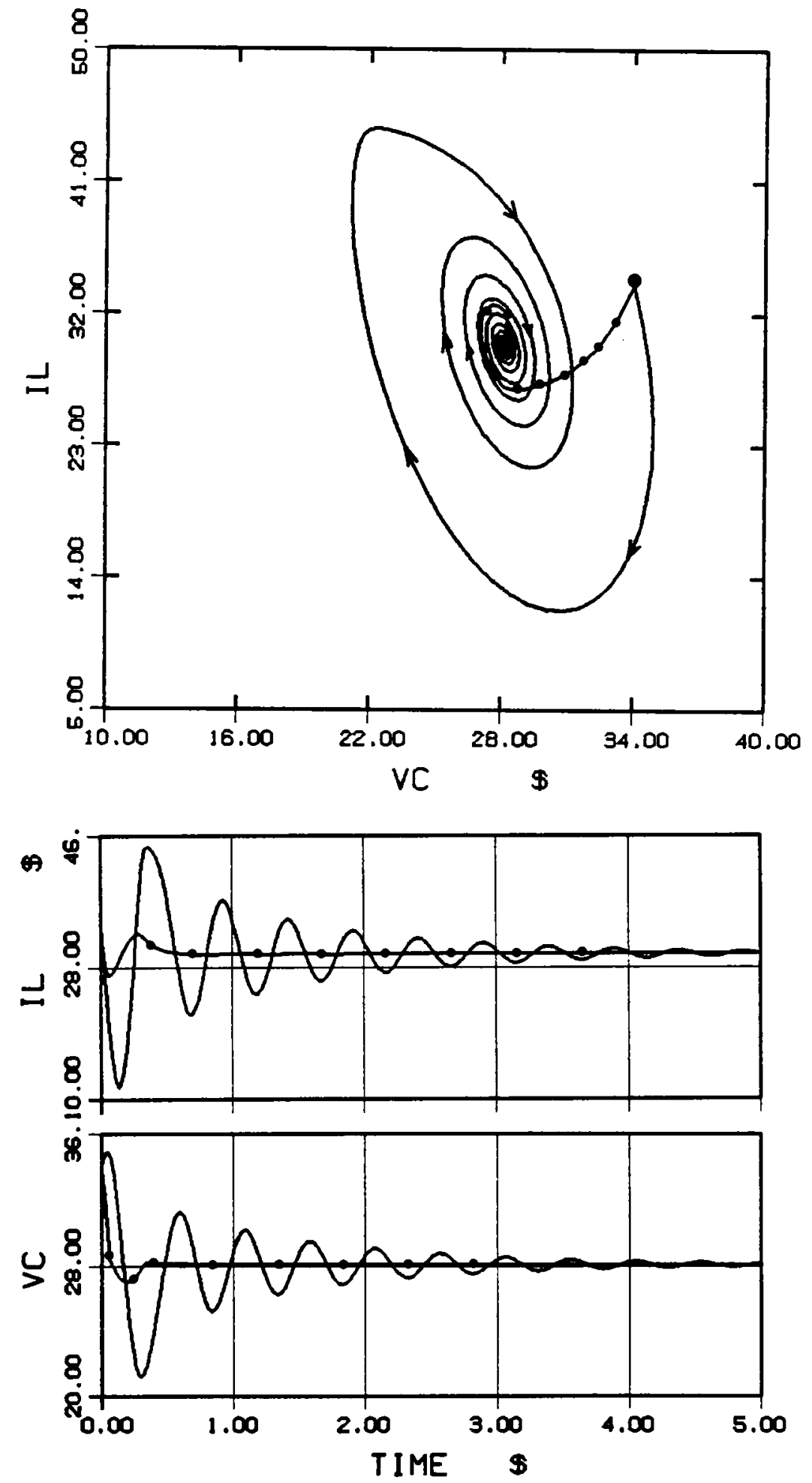

Figure 5.8 Comparison in the system response with and without the shunt $\leftrightarrow$ : with shunt

- without shunt 


$$
\frac{V}{I}=-\frac{\partial V}{\partial I}
$$

Eq. (5.22) states that at the maximum power point, the constant power load line, $R_{L}$, is equal to the dynamic output resistance of the solar array, $R_{0}$, as shown in Fig.5.9.

To analyze the behavior of an equilibrium point, one can use the solar array small signal model depicted by Eq.(3.5). One of the system eigenvalues at the equilibrium point is

$$
\lambda=-\frac{1}{C_{\text {eq }}\left(1+R_{\text {eq }} / r_{L}\right)}
$$

where $\mathrm{C}_{\text {eq }}$ and $\mathrm{R}_{\text {eq }}$ are defined in Eq. (3.61).

From Eq. (5.23), for $r_{L}<0$, the system is:

$$
\begin{aligned}
& \text { stable if }\left|R_{\text {eq }}\right|<\left|R_{L}\right| \text {, and } \\
& \text { unstable if }\left|R_{\text {eq }}\right|>\left|R_{L}\right|
\end{aligned}
$$

Here, $R_{\text {eq }}$ represents the characteristic of the solar array output resistance $r_{S}$ in Eq. (5.9).

In the stable region but near the maximum power point, $M$, the magnitudes of $r_{S}$ and $r_{L}$ are close together, and the eigenvalue is close to the origin, which implies very poor transient response and stability margin. 


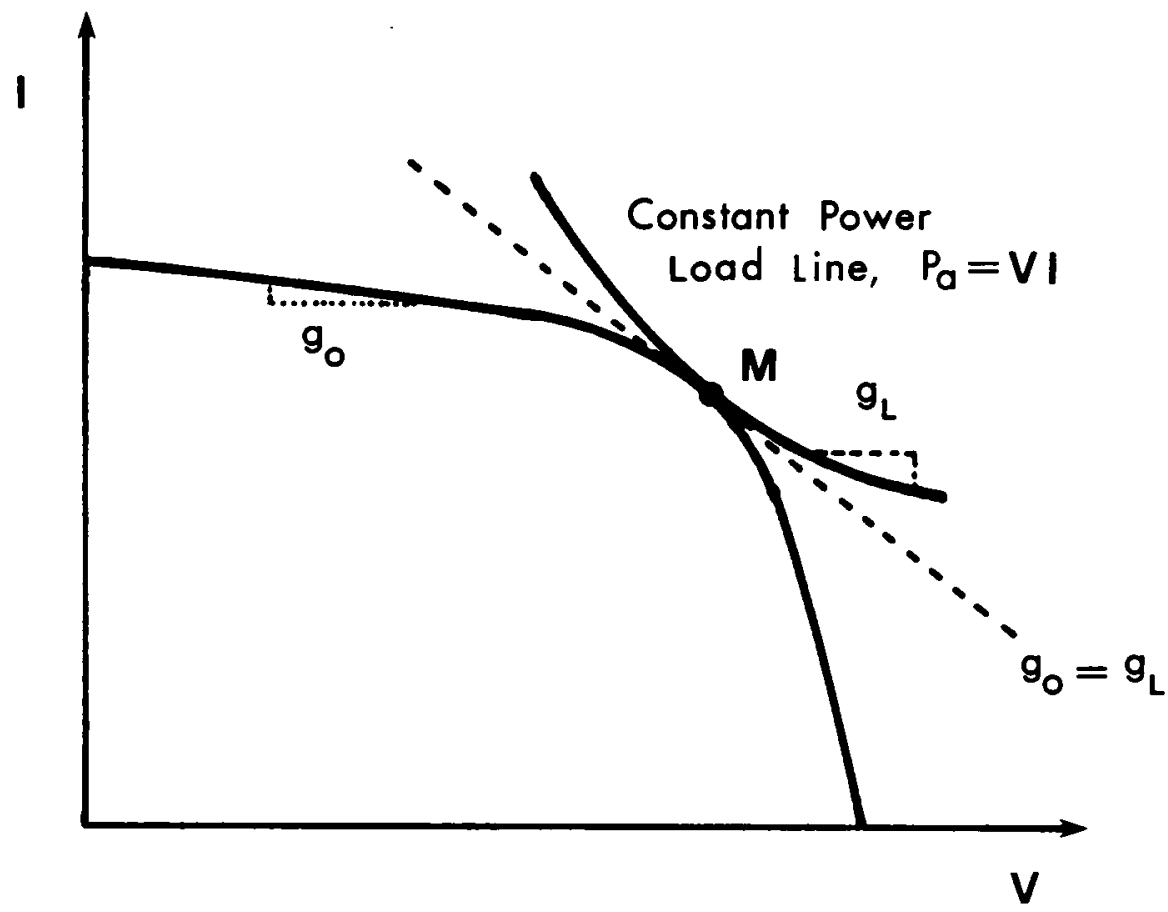

Figure 5.9 Relationships of solar array characteristic and constant power load line at the maximum power point, $M$ 
In this respect it is desirable to operate the system in a region where $\left|r_{S}\right| \ll\left|r_{L}\right|$. This can be seen from the step transient response simulation using the system model developed in Section 5.2 (Fig.5.2). Fig.10a shows the response of the step change in the load power from 800 watts to 790 watts, and Fig.5.10b from 1040 watts to 1030 watts which is close to the maximum power point (1050 watt). Therefore, to design a controller to utilize the maximum available power from the solar array, one must take into consideration of the stability of the operating point and the transient behavior of the system. One way to overcome this problem is to perhaps use the shunt regulator to dissipate some solar array power. In this mode of operation, the system can be stabilized as shown in Section 5. 2 .

\subsection{Analysis of the solar array system in the battery discharge mode.}

The basic functional requirement of the battery discharger is to regulate the bus voltage when the solar array is incapable of meeting the load demand. In this mode both the solar array and the battery function, as power sources. Fig.5.11 shows the system operating in the battery discharge mode. When the bus voltage drops below a certain magnitude, the control signal activates the battery discharge converter in order to maintain regulation of the bus voltage. The solar array then supplies the current corresponding to this regulated bus voltage.

In the DET system, when the solar array output power decreases due to the weakening of the illumination level, the battery discharger operates from no-load condition through the converter discontinuous conduction 

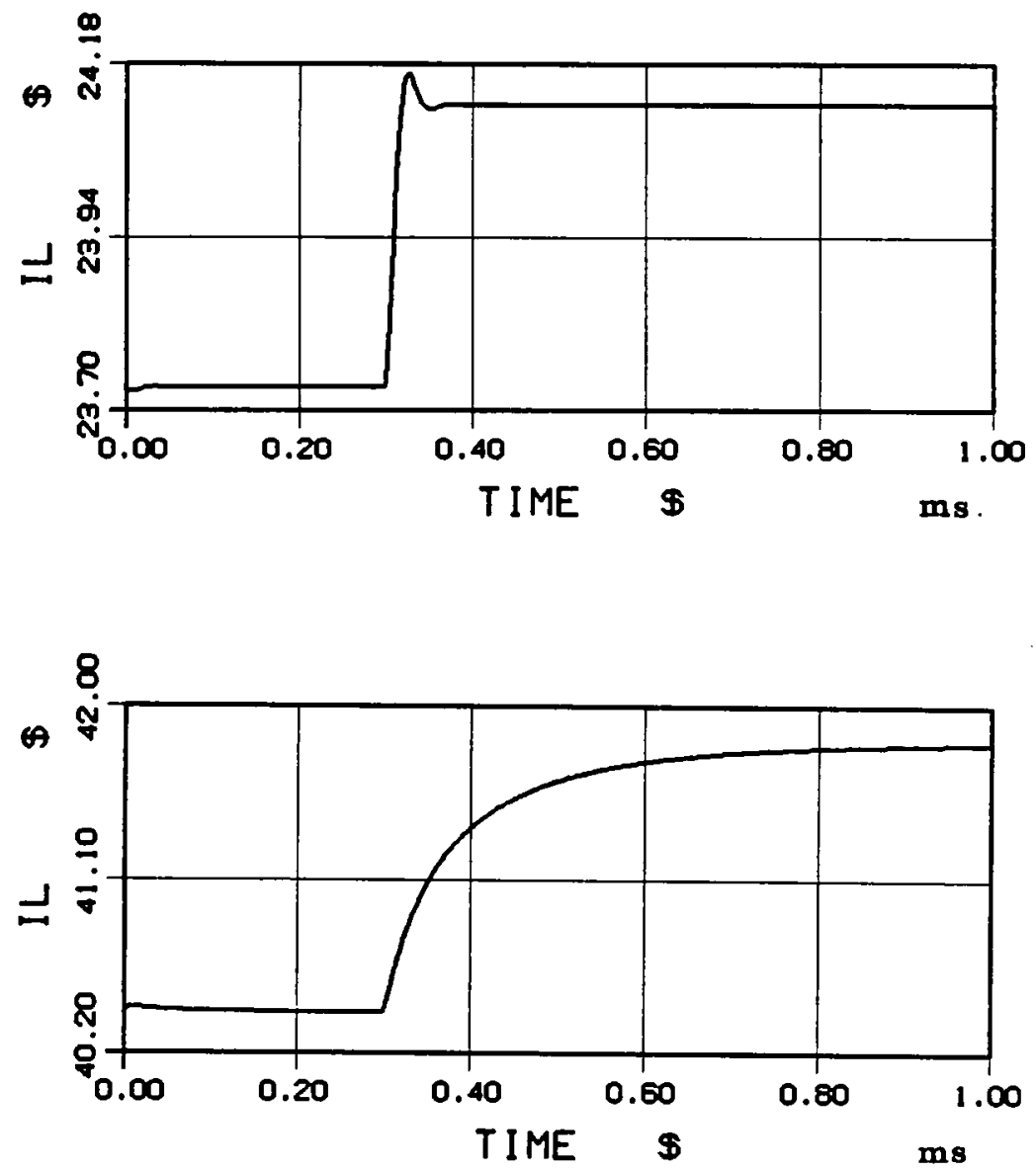

Figure 5.10 Step load transient responses

(a) 800 to 790 watt step

(b) 1040 to 1030 watt step 


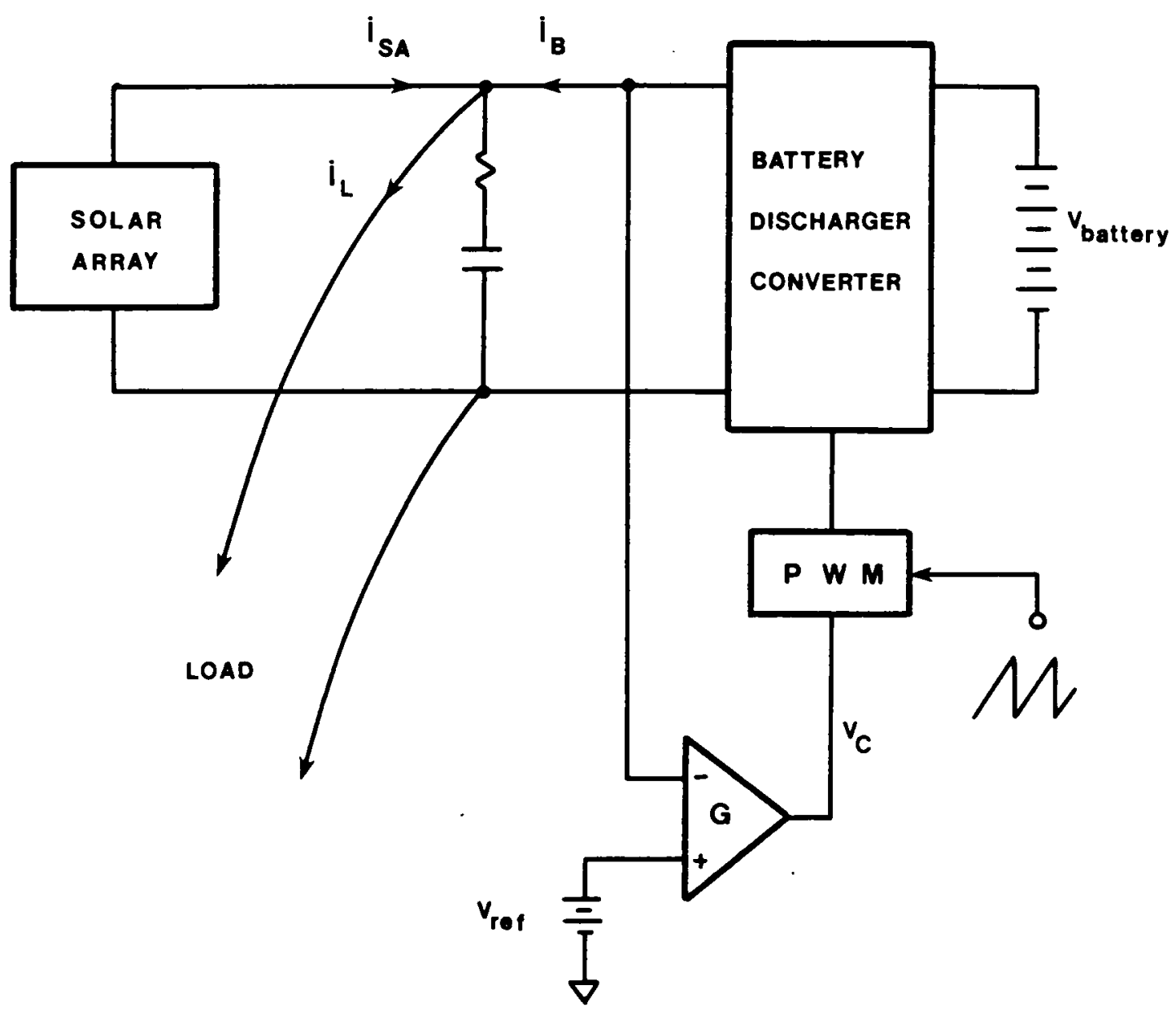

Figure 5.11 System in the battery discharge mode 
mode (DCM) of operation and ultimately to the continuous conduction mode (CCM). Since the dc and ac dynamic behaviors of switching regulators for the two modes of operation are quite different, the analysis and the feedback controller design must take into consideration the two modes of operation.

A particular battery discharge switching converter power stage used in the DET system is shown in Fig.5.12. The circuit basically operates as a buck type regulator operating in push-pull mode. Since the terminal voltage of the battery is between 17 and 25 volts, an auto-transformer is used to boost the voltage to $28 \mathrm{~V}$. In this study, a simplified circuit is used as shown in Fig.5.13a. The two sources in Fig.5.13a represent the transformer action between the power switches ON and OFF intervals.

The state space averaging technique is employed to analyze the converter in the discontinuous conduction mode. As illustrated in Fig.3.2, there exist three intervals $d_{1} T_{s}, d_{2} T_{s}$ and $d_{3} T_{s}$. While the first interval, $d_{1} T_{s}$, (inductor charging) is dictated by the "ON" time of the switching function and is a known quantity, the second interval, $\mathrm{d}_{2} \mathrm{~T}_{\mathrm{s}}$, (inductor discharging) is yet unknown and depends in general on both the length of the first interval and circuit parameters. However, $\mathrm{d}_{2} \mathrm{~T}_{s}$ can be determined using the additional constraint in the discontinuous conduction mode described in Eqs.(3.5) and (3.6)

While the state space expression in Eq. (3.8) was sufficient to describe the converter operating in continuous conduction mode, in discontinuous conduction mode, the state equations for the three switched intervals in 


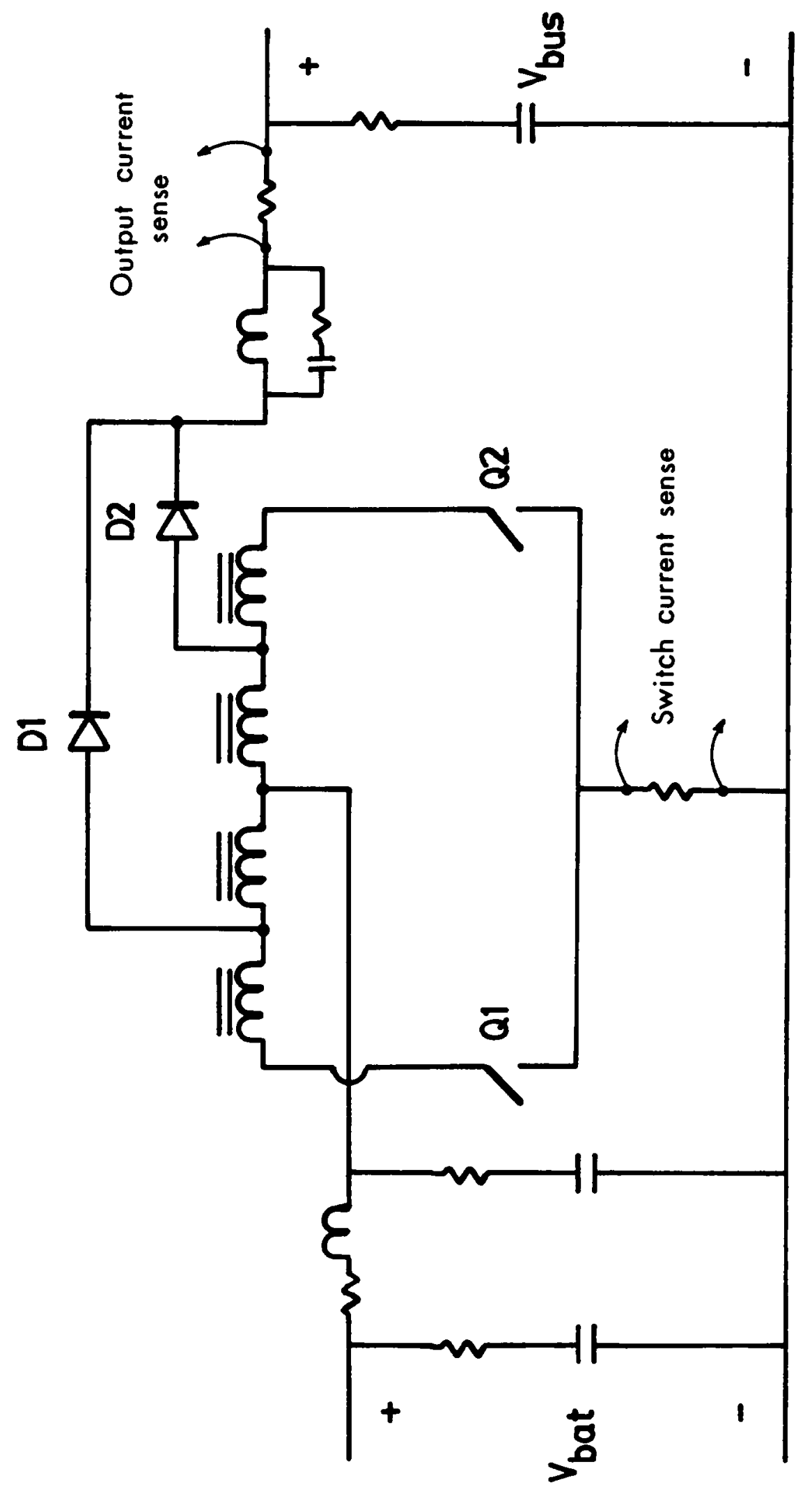

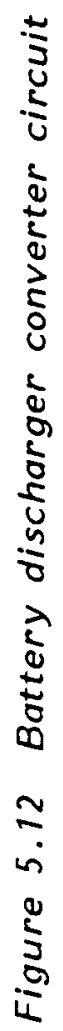




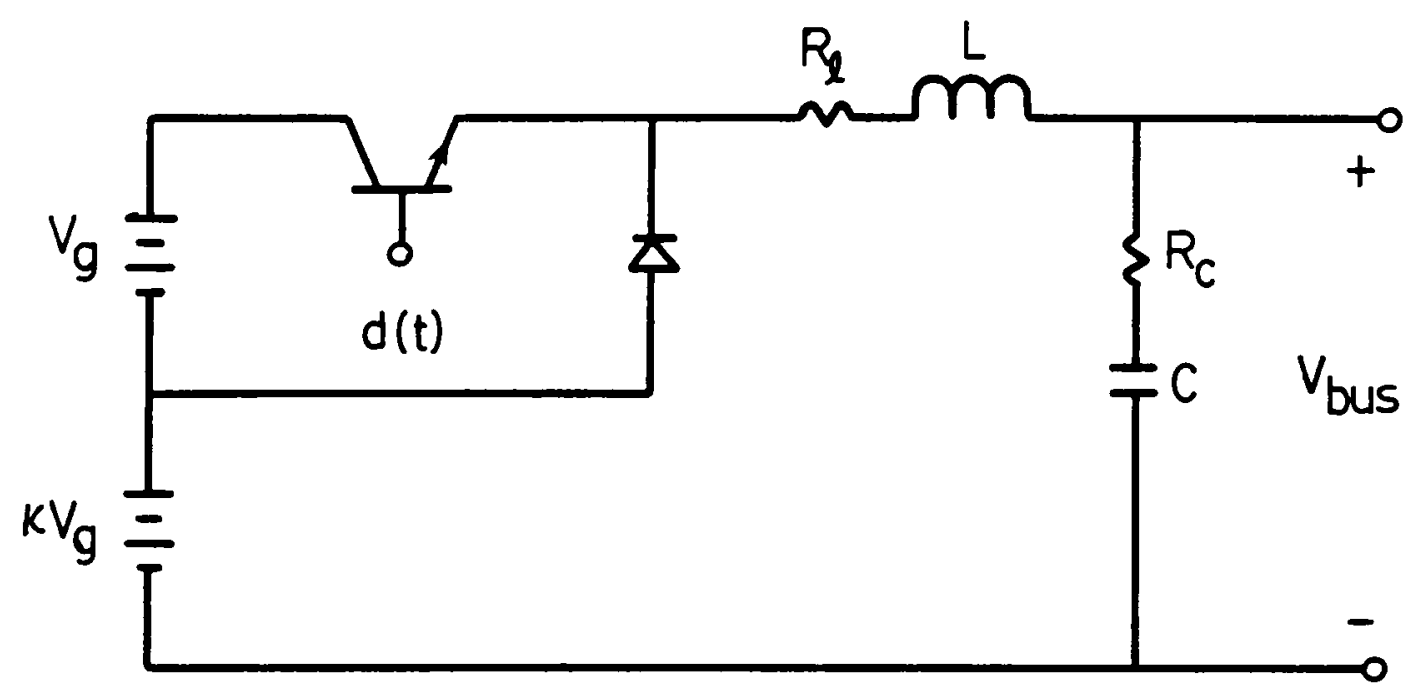

(a)

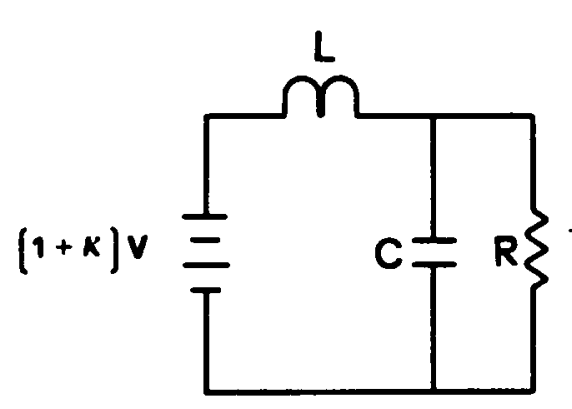

$d_{1} T_{s}$

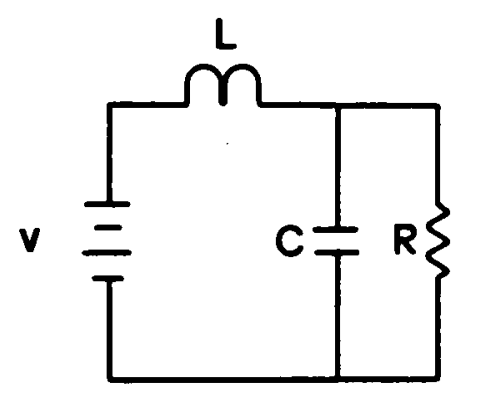

$d_{2} T_{S}$

(b)

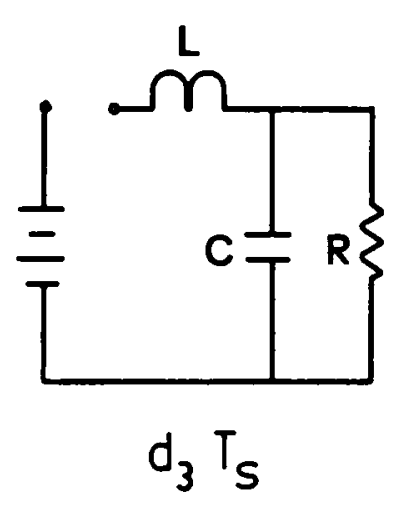

$d_{3} T_{s}$

Figure 5.13 (a) Simplified circuit of the converter in Fig.5.12

(b) Three switched circuits 
Eq. (3.8) do not describe the switching converter completely. Namely, the instantaneous inductor current is restricted in its evolution such that

$$
\begin{gathered}
i(0)=i\left[\left(d_{1}+d_{2}\right) T_{s}\right]=0 \\
i(t)=0 \text { for interval } d_{3} T_{s}
\end{gathered}
$$

The state equations of Eq.(3.8) together with Eq. (5.24) completely determine the behavior of the switching converter. Using ideal elements in the circuit in Fig.5.13a and ignoring load dynamics, three switched networks in the discontinuous conduction mode are shown in Fig.5.13b. For the choice of the state vector $x=\left[\begin{array}{ll}i & v\end{array}\right]^{T}$, the state space equations of the three switched networks becomes:

$$
\begin{aligned}
& \dot{x}=A_{1} x+B_{1} v_{g} \quad \text { for interval } d_{1} T_{s} \\
& \dot{x}=A_{2} x+B_{2} v_{g} \quad \text { for interval } d_{2} T_{s} \\
& \dot{x}=A_{3} x+B_{3} v_{g} \quad \text { for interval } d_{3} T_{s}
\end{aligned}
$$

where

$$
A_{1}=A_{2}=\left[\begin{array}{cc}
0 & -\frac{1}{L} \\
1 & -\frac{1}{C R}
\end{array}\right], \quad A_{3}=\left[\begin{array}{cc}
0 & 0 \\
0 & -\frac{1}{C R}
\end{array}\right]
$$




$$
B_{1}=\left|\begin{array}{c}
\frac{(1+\kappa)}{L} \\
0
\end{array}\right|, \quad B_{2}=\left|\begin{array}{c}
\frac{\kappa}{L} \\
0
\end{array}\right|, \quad B_{3}=0
$$

In addition to this,

$$
i=\frac{i_{\max }}{2}
$$

Eqs. (5.25) and (5.27) contain all that is needed to determine both dc and ac small signal models. Let us first consider the steady state (dc) model.

\section{Steady state (dc) model analys is}

Averaging Eq.(5.26), the following linear algebraic static equations result.

$$
\begin{aligned}
& \left.\left[\begin{array}{cc}
0 & -\frac{D_{1}+D_{2}}{L} \\
\frac{D_{1}+D_{2}}{C} & -\frac{1}{C R}
\end{array}\right]\left[\begin{array}{c}
I \\
V
\end{array}\right]+\left[\begin{array}{c}
\frac{D_{1}(1+K)+K D_{2}}{L} \\
0
\end{array}\right] \begin{array}{c}
V=0 \\
g
\end{array}\right] \\
& \mathrm{A} \cdot \mathrm{X}+\mathrm{B} \cdot \mathrm{V}_{\mathrm{g}}=0
\end{aligned}
$$

Then the steady state solution of Eq. (5.28) is 


$$
\begin{gathered}
\frac{V}{V_{g}} \equiv M=\frac{D_{1}(1+K)+k D_{2}}{D_{1}+D_{2}} \\
I=\frac{V}{R} \frac{1}{\left(D_{1}+D_{2}\right)}
\end{gathered}
$$

Hence, the dc conditions depend not only on $D_{1}$ and $R$, as in the continuous conduction mode, but also on $\mathrm{D}_{2}$. By use of the additional constraint in the average of inductor current for $\left(D_{1}+D_{2}\right) T_{s}$,

$$
I=\frac{(1+K) V_{g}-V}{2 L} D_{1} T_{s}
$$

the dc conditions are completely determined with the known quantities $D_{1}$ and $R$. For example, substitution of Eq.(5.30) into Eq.(5.28) results in

$$
D_{2}=\frac{1}{2}\left[\left(\frac{K \tau}{D_{1}}-D_{1}\right)+\left[\left(\frac{K \tau_{n}}{D_{1}}-D_{1}\right)^{2}+4 \tau_{n}(1+K)\right]^{\frac{1}{2}}\right]
$$

where

$$
\tau_{\mathrm{n}} \equiv 2 \mathrm{~L} /\left(\mathrm{R} \mathrm{T} \mathrm{T}_{\mathrm{S}}\right) ; \text { normalized time constant. }
$$

The boundary condition between the two conduction modes is

$$
\begin{aligned}
& \mathrm{D}_{2}<1-\mathrm{D}_{1} ; \text { discontinuous conduction mode } \\
& \mathrm{D}_{2}>1-\mathrm{D}_{1} ; \text { continuous conduction mode }
\end{aligned}
$$


Using Eqs.(5.31), the critical value of $\tau_{n}$ can be determined by solving $\mathrm{D}_{2}=1-\mathrm{D}_{1}$.

$$
\tau_{\text {ncrit }}=\frac{1-D_{1}}{1+k+k\left(1-D_{1}\right) / D_{1}}
$$

Suppose now that the output voltage is regulated with some proper feedback circuit, then the dc voltage gain $M$ can be treated as a constant for a fixed input voltage. The duty ratio, $D_{1}$, in the discontinuous conduction mode can be derived as a function of $M$, or as a function of $\tau_{n}$.

$$
D_{1}=\left[\frac{\tau_{n} M(M-k)}{1+k-M}\right]^{\frac{1}{2}}
$$

Using the results in Eqs. (5.29) through (5.34), the dc behavior of the battery discharger is illustrated in Fig.5.14. In Fig.5.14a, the boundary condition, ' $\tau_{\text {ncrit }}$, between the two modes is plotted as a function of $D_{1}$. The dc voltage gain, $M$, as function of $D_{1}$ for different values of $\tau_{n}$ is shown in Fig.5.14b.

Since the battery discharger operates from no-load condition to fullload, it is important to understand the start-up behavior of the converter. Suppose now that the solar array output power gradually decreases. Initially the battery discharging current is zero (i.e., $\tau_{n}=0$ ) and the duty ratio $D_{1}=0$. As the solar array output power decreases, the operating point moves along the solar array curve which causes the bus 

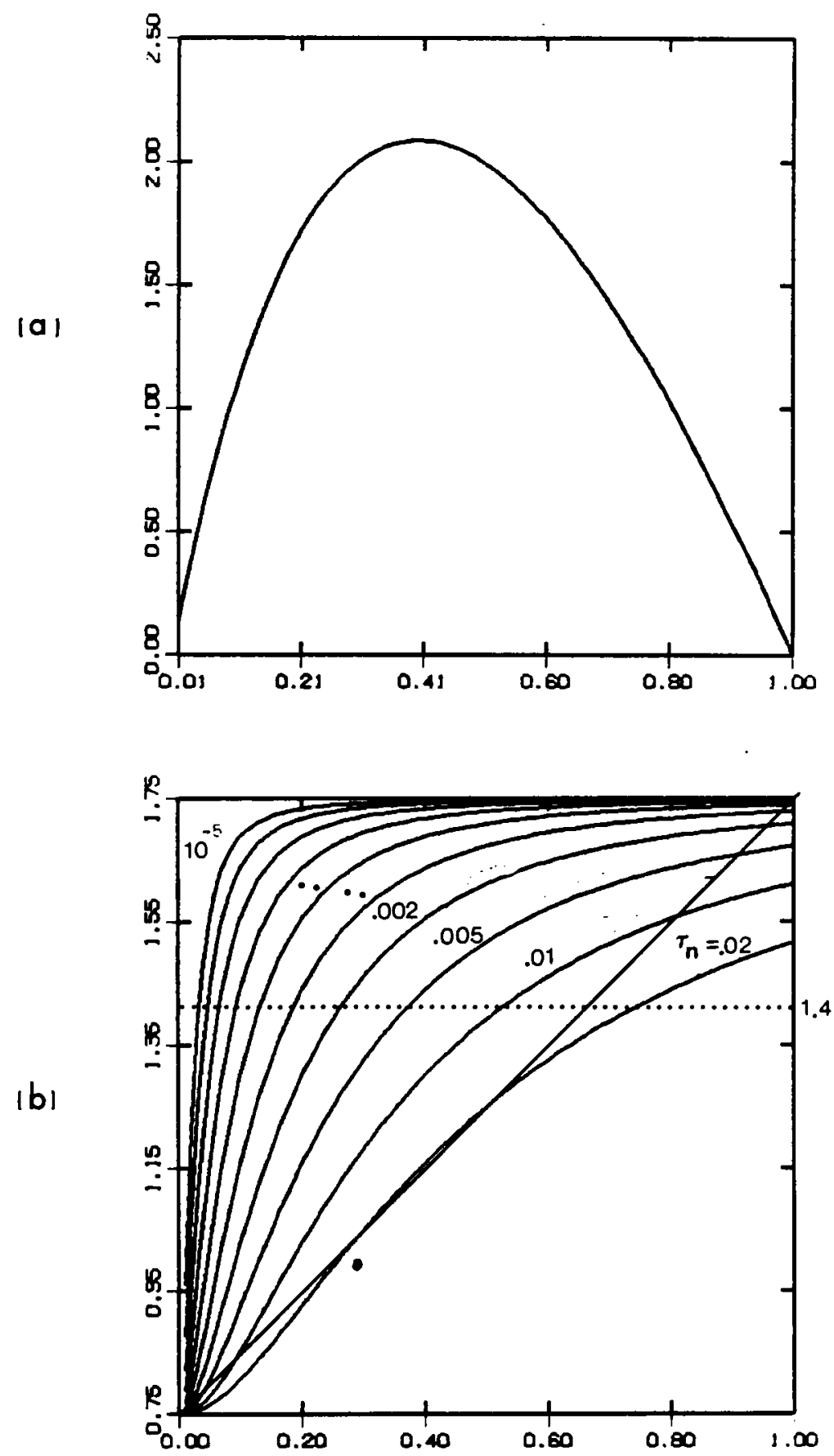

Figure 5.14 DC characteristics of the battery discharger converter
(a) $\tau_{\text {ncrit }} \vee s D_{1}$
(b) $M \vee s D_{1}$ 
voltage to drop, thus activating the battery discharger electronics via an error amplifier. In this start-up mode, it is very important to design a proper error amplifier circuit for the following reasons.

For the high gain controller, since the sensitivity of the dc voltage gain with respect to the duty ratio is very high as shown in Fig.5.14b, any delay in the logic circuitry or the false triggering due to the noise will cause a high frequency oscillation in the bus voltage. While in a low feedback gain system the controller may not act fast enough to regulate the bus voltage, since the plant time constant of the output capacitor and load resistance is very slow. This may cause a large amplitude, low frequency oscillation It is especially critical when the output voltage of the battery discharger converter is set such that its operation is close to the solar array maximum power point. Since the low gain controller allows the bus voltage to drop before the controller starts correcting the error, it is possible that the system may move into the unstable region as discussed previously. Another important concern when designing the error amplifiers is that, since the battery discharger operates from the discontinuous conduction mode to continuous conduction mode, the error amplifier should be able to stabilize the system in both modes. Thus, it is necessary to analyze the dynamic behavior of the converter in the discontinuous conduction mode. 
Small-signal analysis: Perturbation and linearization of the averaged equations of (5.25) and (5.27) result in the following ac dynamic model.

$$
\begin{aligned}
& {\left[\begin{array}{c}
\hat{i} \\
\hat{v}
\end{array}\right]=\left[\begin{array}{cc}
0 & -\frac{D_{1}+D_{2}}{L} \\
\frac{D_{1}+D_{2}}{C} & -\frac{1}{C R}
\end{array}\right]\left[\begin{array}{c}
\hat{i} \\
\hat{v}
\end{array}\right]+\left[\begin{array}{c}
\frac{D_{1}(1+K)+K D_{2}}{L} \\
0
\end{array}\right] \hat{v}_{g}} \\
& +\left[\frac{(1+K) V_{g}-V}{L}\right] \hat{d}_{1}+\left[\begin{array}{c}
\frac{k V_{g}-V}{L} \\
I \\
C
\end{array}\right] \hat{d}_{2}
\end{aligned}
$$

with additional constraints

$$
\begin{gathered}
\frac{d \hat{i}}{d t}=0 \\
i=\frac{I}{D} \hat{d}+\frac{(1+K) I}{(1+K) V_{g}-V} \hat{v}_{g}-\frac{I}{(1+K) V_{g}-V} \hat{v}
\end{gathered}
$$

Substituting the additional constraints from Eqs.(5.36) and (5.37) into Eq. (5.35), the unknown modulation quantities $d_{2}$ and $i$ can be determined in terms of other known dc and ac quantities. Thus, the complete dynamic model can be expressed as 


$$
\begin{aligned}
& C \frac{d \hat{v}}{d t}=\left[\frac{1}{R} \frac{(2 M-K)-(K-M)^{2}}{(1+K-M)(K-M)}\right] \hat{v} \\
& +\left[\frac{M}{R} \frac{M D_{2}+D_{1}+K D_{1}}{(1+K-M)(K-M)\left(D_{1}+D_{2}\right)}\right] \hat{v}_{g} \\
& +\left[\frac{V}{R} \frac{\left(D_{1}+D_{2}\right)(K-M)-D_{1}}{D_{1}\left(D_{1}+D_{2}\right)(K-N)}\right] \hat{d}_{1}
\end{aligned}
$$

It is now easy to obtain from (5.38) the two transfer functions of interest

where

$$
\begin{aligned}
& \frac{\hat{v}}{\hat{v}_{g}}=G_{\circ g} \frac{1}{1+s / \omega_{p}} \\
& \frac{\hat{v}}{\hat{d}_{1}}=G_{\text {od }} \frac{1}{1+s / \omega_{p}}
\end{aligned}
$$

$$
\begin{gathered}
G_{\circ g}=M \\
G_{\text {od }}=V \frac{\left(D_{1}-\left(D_{1}+D_{2}\right)(K-M)\right)(1+K-M)}{D_{1}\left(D_{1}+D_{2}\right)\left((2 M-K)-(K-M)^{2}\right)} \\
\omega_{p}=\frac{1}{R C} \frac{(K-M)^{2}-(2 M-K)}{(1+K-M)(K-M)}
\end{gathered}
$$


The transfer function models are rather complicated for this particular converter circuit, however, they are expressed in terms of well defined constants which can easily be calculated. The qualitative interpretations of the results are as follows.

Eqs. (5.39) and (5.40) show that the transfer functions have a single pole and no zero. Due to the additional constraint in Eq. (5.36), any converter that can be described with two state variables of which one is an inductor current, has a single pole system transfer functions in the discontinuous conduction mode. Thus, the dynamic behavior of the converter operating in the discontinuous conduction mode is completely different from that of the continuous conduction mode, in which the system has a complex pole pair. Therefore, the feedback controller should compensate the plant dynamics in both modes of operation. This is clearly illustrated in the root locus diagram of the open loop converter as a function of the load resistance in Fig.5.15. Without a compensator, the system eigenvalue is close to the origin, thus the system response should be very slow in the discontinuous conduction mode as intuitively predicted in the dc analysis. Decreasing the load resistance beyond $R_{c r i t}$, the converter will operate in the continuous conduction mode, and the open loop eigenvalues become complex. In this range, the response is still slow and oscillatory. A further decrease of the load resistance will move the locus onto the circle with the radius of $\omega_{0}=\sqrt{\mathrm{LC}}$, and finally the locus approaches to the real axis again. 


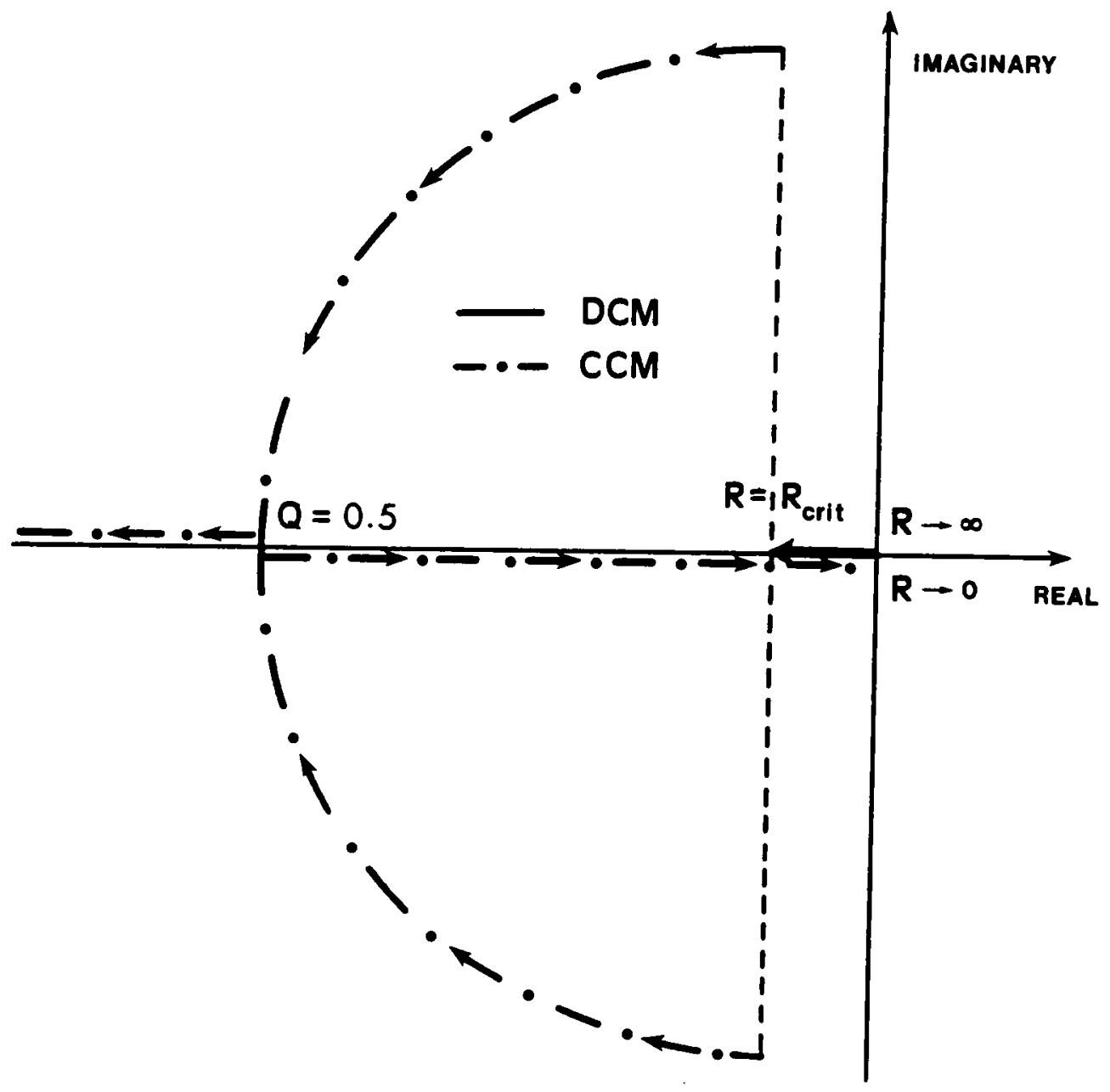

Figure 5.15 Root locus of the open loop buck-type converter as a function of load resistance, $R$ for DCM and CCM 
Fig.5.16 illustrates the root locus diagrams for different compensators for an arbitrary load resistance in both DCM and CCM. Let us first consider a compensator for the converter operating in the DCM. In order to compensate the open loop single pole near the origin as described in Eqs.(5.39) through (5.43), a simple proportional gain controller can be used to move the eigenvalue along the negative real axis to speed up the response, as shown in Fig.5.16a. However, this gain also moves the eigenvalues to the imaginary axis in the CCM and it is worse in a light-load condition in which the open loop poles are already close to the imaginary axis. A low gain may stabilize the system in the CCM, but the slow response in the DCM may cause severe bus voltage droop.

A typical integrator with lead-lag compensators as illustrated in Fig.5.16b is examined next. The integrator is used for the tight regulation of the output voltage, the zero compensates the excessive phase lag due to the presence of an integrator, and the pole attenuates the high frequency noise entering the controller. However, the integrator slows down the system response in DCM as shown in the figure, and the similar situation will occur as the low gain in the above case. In fact, for an optimum selection of the feedback gain for the regulator in CCN, the locus still stays near the imaginary axis in DCM.

Therefore, to achieve an optimum response for the both modes of operations, a compensator for switching converters in the battery discharger application must include a proportional gain to speed up the system response in DCM and the lead-lag type compensator for stable operation in CCM. This is illustrated in Fig.5.16c. 

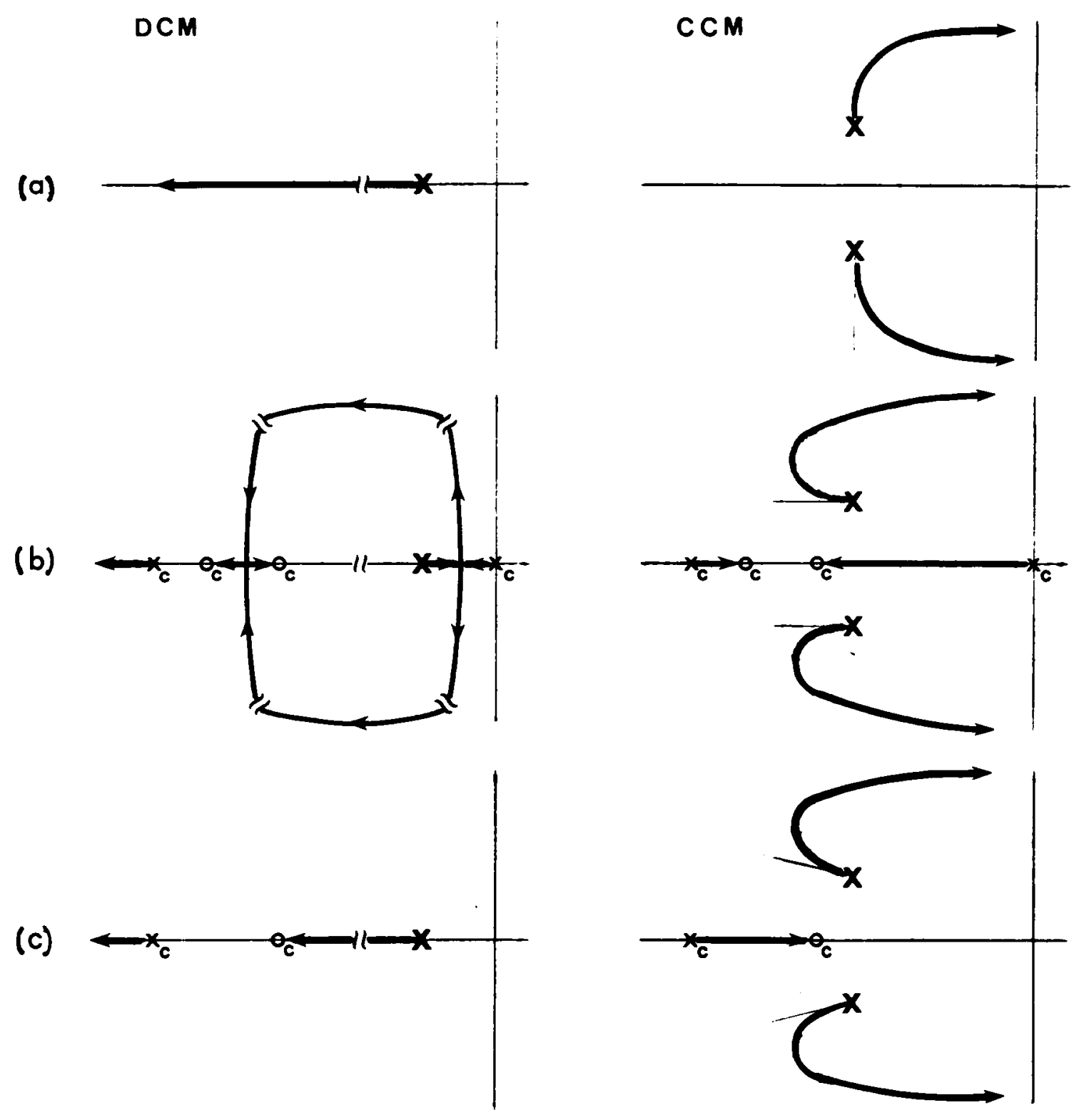

Figure 5.16 Root loci of the closed loop buck-type converter in DCM and CCM for three compensators

(a) proportional gain

(b) integrator plus lead-lag

(c) proportional plus lead-lag

$$
\begin{aligned}
& x_{c} \text { : compensation pole } \\
& o_{C} \text { : compensation zero }
\end{aligned}
$$


In order to verify the above analysis and to observe the effect of the integrator in the DCM, the DET system is simulated employing the compensators shown in Fig.5.17. Fig.5.18 shows the step load transient response (from 1300W to 1400W) of the system when the battery discharger operates in the continuous conduction mode. As shown in the figure, the system behaves equally well for both compensators. In Fig.5.19, the load power ( $i_{\text {load }}$ ) is gradually increasing and various modes are illustrated. The system initially operates in the shunt mode until $0.6 \mathrm{~ms}$, then it gets into the dead band mode where the solar array solely supplies the load power. The operating point moves along the solar array curve as the load power increases. The battery discharger employing the compensator with the proportional gain (Fig.5.16c) starts regulating the bus voltage at $t=1 \mathrm{~ms}$ as shown in Fig.5.19a. While that using the integrator starts to operate at $t=2 \mathrm{~ms}$ and $\mathrm{slowly}$ increases to the regulation voltage as shown in Fig.5.19b. As shown in this figure, the slow response in DCM of the integrator type controller may cause the bus voltage to drop out of the regulation range.

The results in Figs.5.16 and 5.19 show that a compensator designed for CCM mode of operation may not be suitable for DCM mode of operation, and consequently, may result in a bus voltage oscillation or large voltage droop due to a load transient. Design of a compensator for the boost or buck-boost type converter must be done more carefully. This is because such converters also have a single pole in the discontinuous conduction mode, but, in the continuous conduction mode, there are a complex pole pair and a right-half plane zero. 


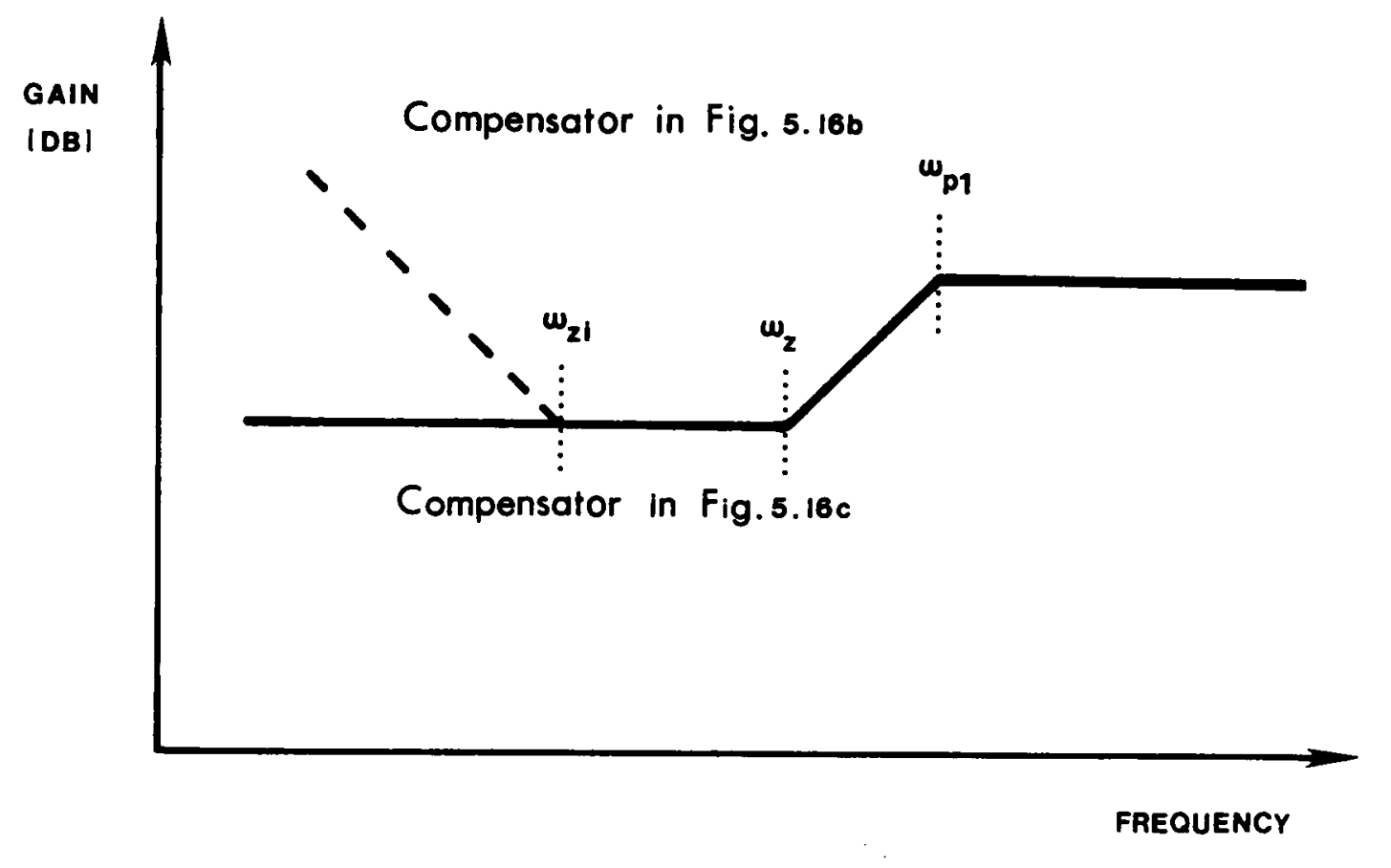

Figure 5.17 Two compensators used for the simulation

$$
\text { Power stage : } \begin{aligned}
\quad \omega_{0} & =3000 \text { in CCM } \\
& 0<\omega_{p}<12.4 \text { in } D C M
\end{aligned}
$$

$$
\text { Compensator : } \quad \begin{aligned}
\omega_{z i} & =1000 \\
\omega_{z 1} & =6000 \\
{ }^{\omega_{p 1}} & =50000
\end{aligned}
$$




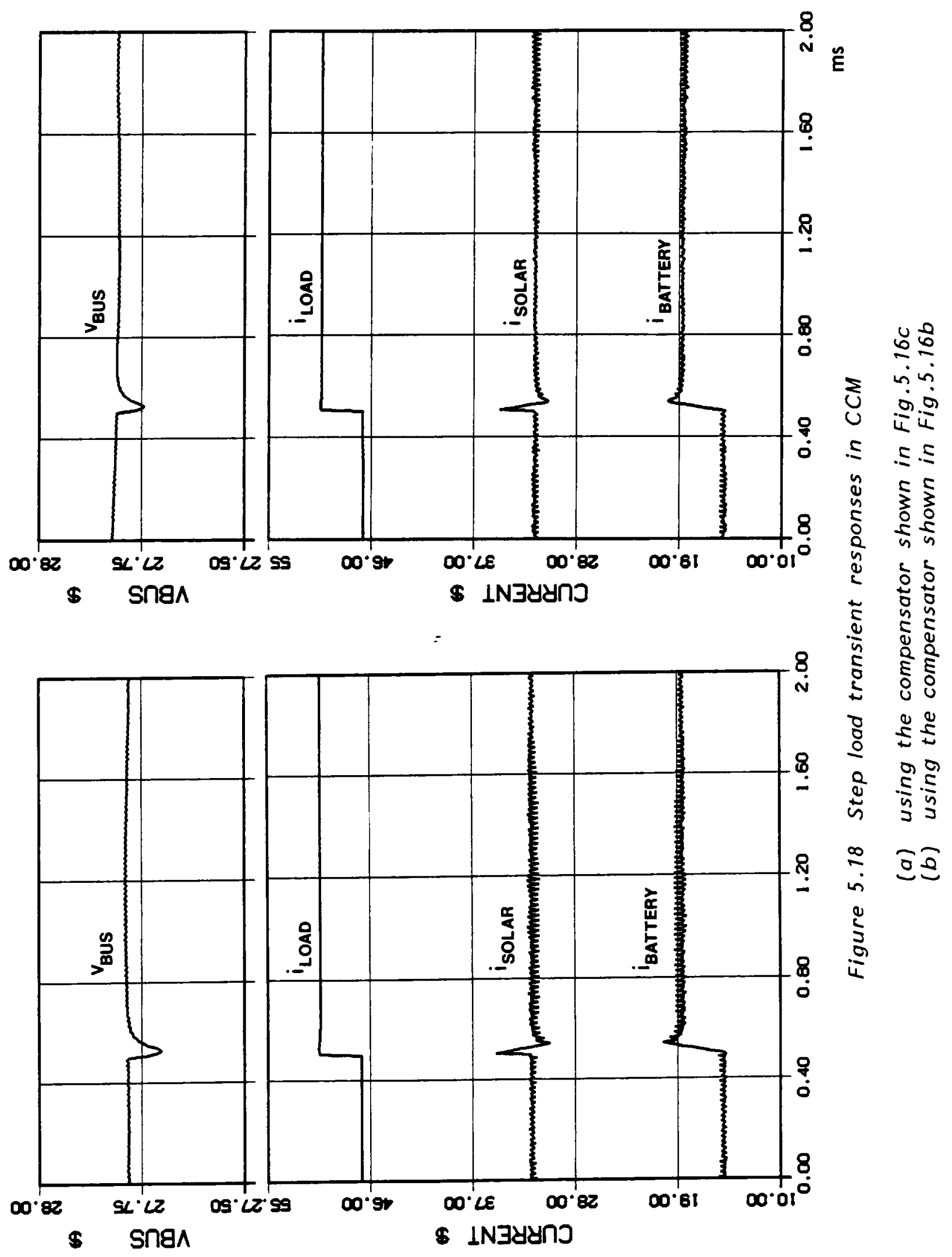



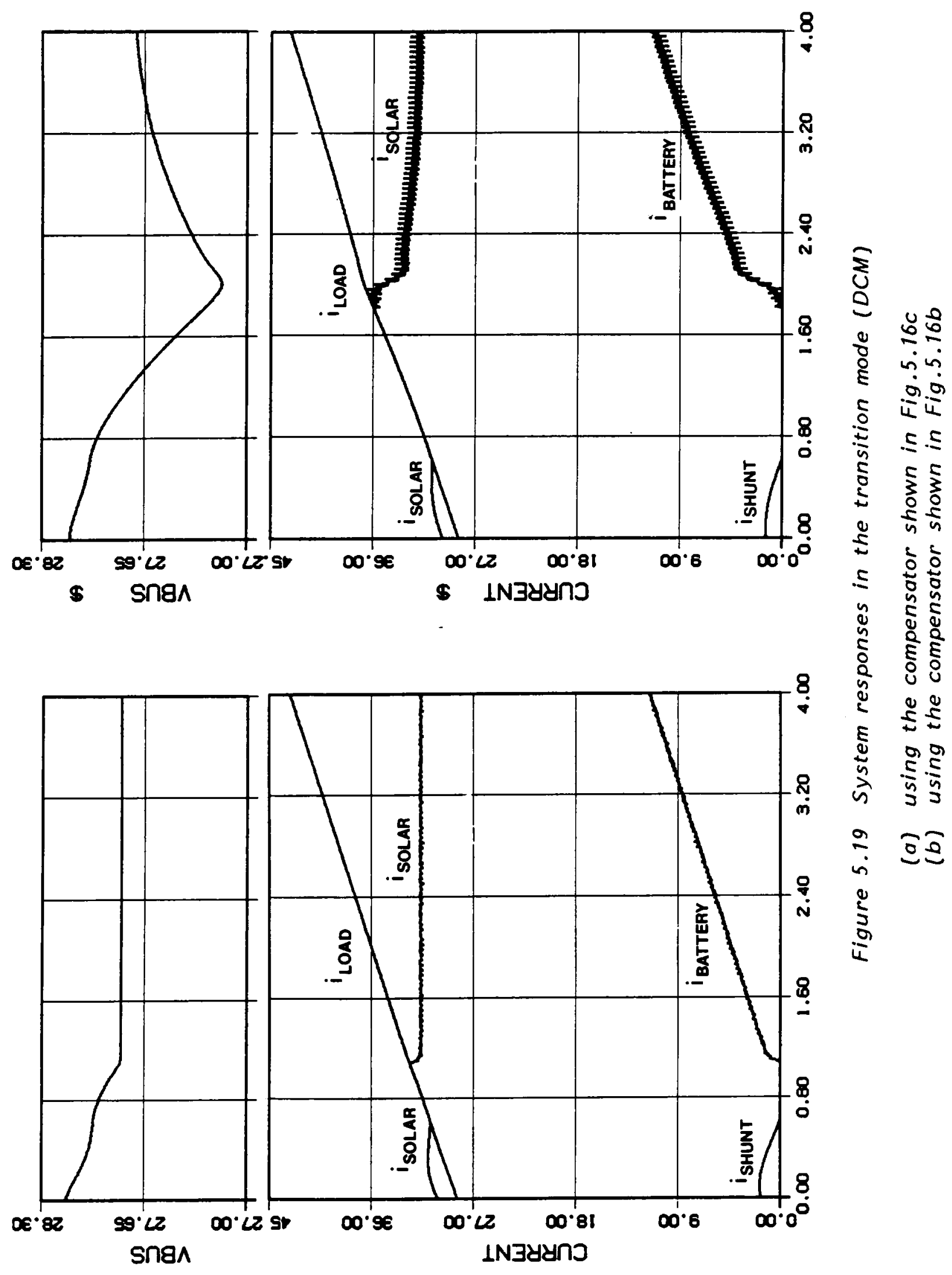


\subsection{Analysis of the solar array system modes of operation}

The modes of operation of the DET system is described in this section. In Fig.4.1 the Central Control Unit (CCU) continuously monitors the bus voltage and commands the system to operate in the battery discharging mode when $v_{\text {bus }}$ is less than $v_{r b}$ or in the shunt active mode when $v_{\text {bus }}$ is greater than $V_{r s}$. When $v_{\text {bus }}$ is between $V_{r b}$ and $V_{r s}$, there exists a "dead band" at which the load power is equal to solar array output power. When the system operates in the shunt active mode, any variation of the bus voltage generates a proportional shunt current, $i_{s h}$. The shunt current together with the load current sinks the solar array current and regulates the bus voltage. When the system operates in the battery discharge mode, the bus voltage is regulated by the battery discharger. The solar array current is set by the bus voltage on its output $I-V$ curve and the battery discharging current is the difference between the solar array current and the load current.

The system operating condition is subject to change due to constantly varying load power consumption and the solar array power generations. The entire DET system shown in Fig.4.1 is simulated to observe how the system operating point moves from one mode to another. Fig.5.20 illustrates the case in which the solar array I-V characteristic varies according to the illumination level. In this example, the illumination level is set to be linearly time varying from Curve 非 (shunt active mode) to Curve $\#_{4}$ (battery discharging mode) as illustrated in Fig.5.20. 


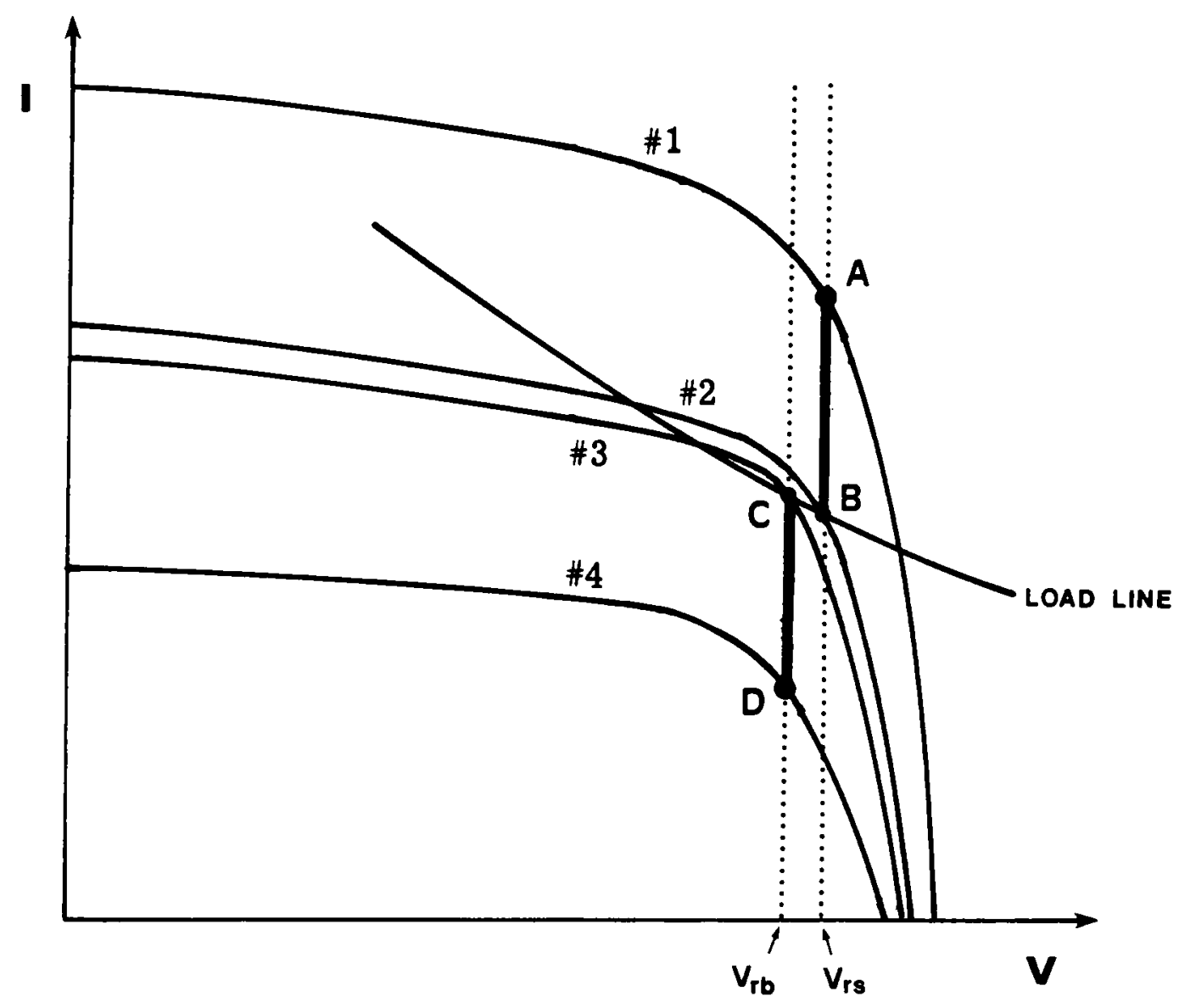

Figure 5.20 DET system mode of operation for the solar array illumination level changes 
From Fig.5.20 and the simulation result in Fig.5.21, the system mode of operation is described as follows: Starting from Curve $\# 1$ and the corresponding operating point $A$, the operating point travels along the shunt regulation line, $V_{\text {rs }}$ until the solar array power decreases to Curve \#2. In this mode, the shunt regulator is active and it absorbs the excessive current generated by the solar array through the shunt elements $\left(i_{\text {sh }}\right)$. Between Curve $\# 2$ and $\#_{3} 3$ the dead band mode exists where both the shunt regulator and battery discharger are deactivated. Thus, the operating point travels along the load line from point $B$ to $C$. Further decrease in the illumination level activates the battery discharger. The operating point then follows the battery discharger regulation line, $\mathrm{V}_{\mathrm{rb}}$. In this mode, the load power is supplied by the solar array and the battery.

Fig.5.22 illustrates the case where the load power is varying while the solar array $I-V$ curve is fixed. With the solar array curve fixed and the load line below Curve $\#^{2}$, the operating point is set at Point A. As the load power increases beyond Curve 非, the shunt regulator is deactivated, and the operating point travels on the solar array curve until Point B. Further increase in the load power beyond Curve $\$ 3$ activates the battery discharger, and the operating point moves along the battery discharger regulation line to Point C. 

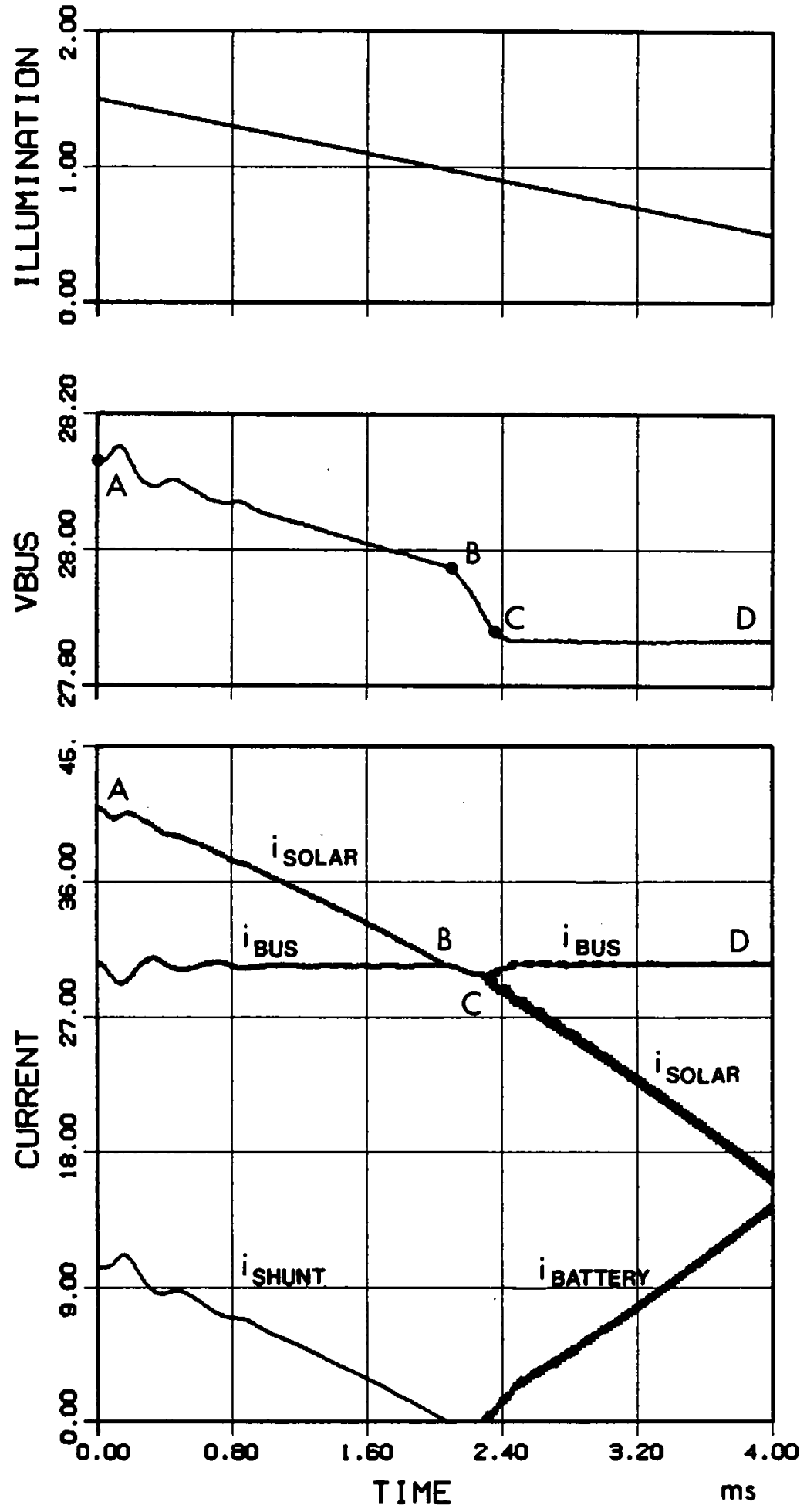

Figure 5.21 Simulation of the DET system for the solar array illumination changes in Fig. 5.20 


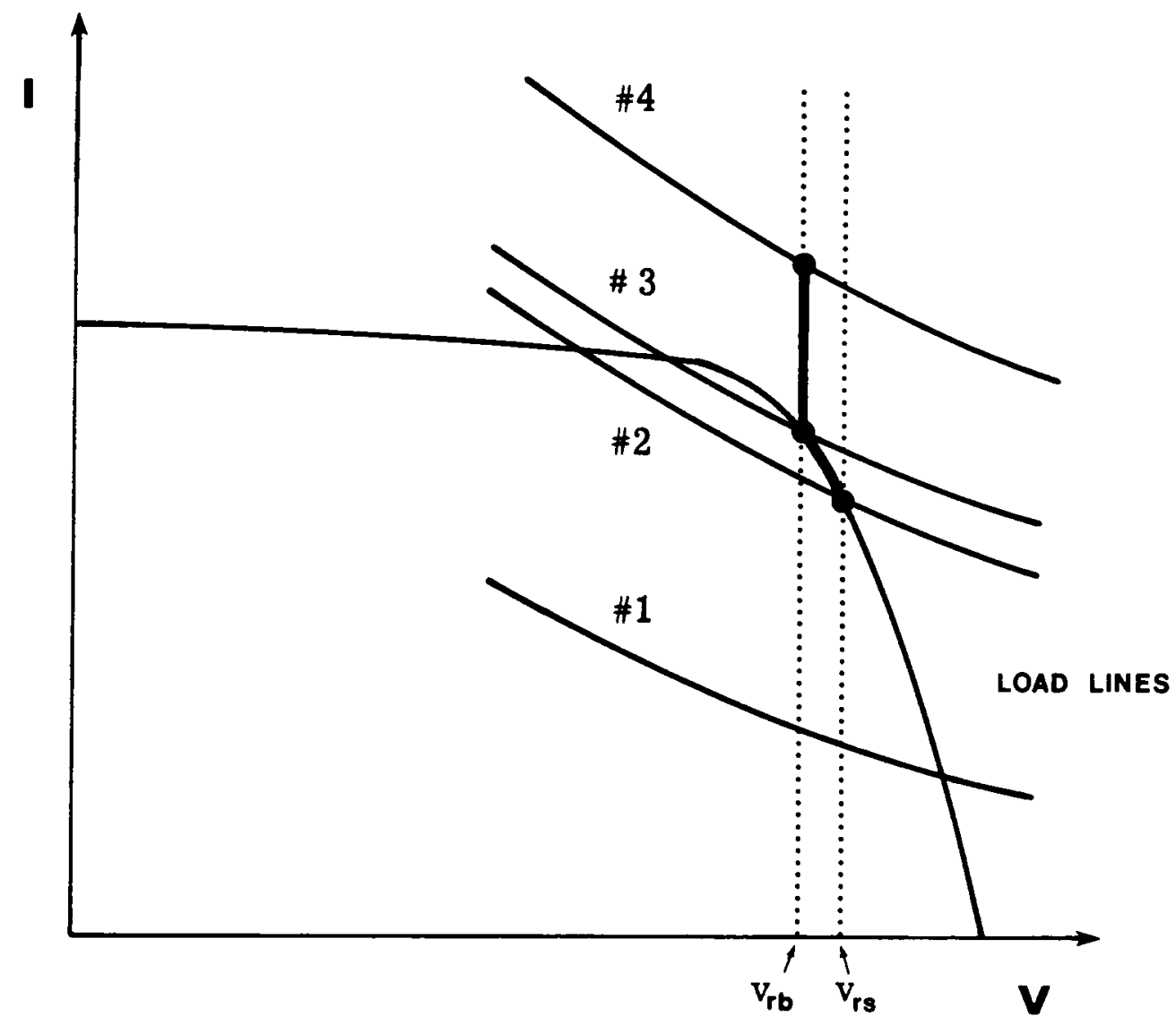

Figure 5.22 DET system mode of operation for the load power changes 


\subsection{Conclusions}

In this chapter, various solar array system modes of operation in the large signal sense are analyzed using the state plane method. The analysis shows that any equilibrium point in Region $\left.\right|_{1} 2$ of Fig.5.1 is unstable with real eigenvalues. The state plane trajectories are separated by the separatrix that passes through Region $\equiv_{1} 2$ and converges to either of the two stable equilibrium points in Region 非 1 or 非3. Thus the behavior of the system is quite different on either sides of the separatrix. Since only the equilibrium point in Region 3 is the desirable operating point, a control method to move the operating point from Region $\left.\right|_{1} 1$ to Region 非 is discussed. The analysis also provides a guide line for the system initial start-up condition that leads to the desired mode of operation.

The behavior of the operating point near the solar array maximum power point is analyzed. As far as stability and transient response are concerned, the equilibrium points near the maximum power point, in general, are less commendable than in the region where the magnitude of the solar array output resistance is small, or in the range of a stiffer voltage source.

Since the battery discharger in the DET system operates from the noload condition through the discontinuous conduction mode (DCM) to the continuous conduction mode (CCM), the feedback compensator must be designed carefully to ensure stability in both modes of operation. Various compensators are compared. The results show that a proportional gain control is most appropriate for the converter operating in the discon- 
tinuous conduction mode. It not only speeds up the system response but also prevents system oscillation near the no-load condition. In the CCM mode of operation, a phase lead type compensator is required to compensate the excessive phase lag due to the complex pole.

The solar array system modes of operation including the shunt regulator, battery and switching regulator load are described. The entire DET system is simulated for a continuously varying illumination level and the behavior of the system in various modes of operation are observed. 


\section{Chapter 6}

\section{SUBSYSTEM INTERACTION ANALYSIS}

\subsection{Introduction}

One of the major difficulties in large scale system analys is involves the subsystem interaction problems. As a system becomes more complex, the interaction problems create a large degree of uncertainties to the system response even though each component may be well understood and documented. Thus, the total system analysis must include the interaction analysis that allows one to predict the system level responses and to trouble-shoot the system. The interaction analysis also provides design information of a component or subsystem at the initial design phase and for the future modification of the system.

As discussed in Chapter 2, the entire system can be considered as a doubly terminated two-port network for a component or subsystem. Thus, in general, the interaction problem can be defined as the interactions among an undetermined component and its terminating subsystems. For the component of interest, these terminating subsystems can be treated as the source and load impedances. The system performance parameters such as stability, audiosusceptibility, etc., are then dependent on the characteristics of these terminating subsystems.

Based on the analysis in Chapter 2, the interaction problems in spacecraft power systems are illustrated using the switching regulator examples. 
Regarding the interaction problem the switching regulator is one of the most critical components in the spacecraft power systems. This is because the switching regulator's feedback loop gain characteristic depends largely on the characteristics of connected source and load impedances. Also, its power stage energy storage elements, $L$ and $C$, generate resonance in the system that can cause peaking of the input or output impedance and give an undesired interaction. It is especially critical in the source interaction case because of the negative input impedance characteristic of switching regulator at low frequencies.

As stated in Chapter 3, it is better to consider the load and source interactions separately to obtain analytical insight into the system. In Section 6.2, the load interaction problem is discussed using an ideal source. In Section 6.3 , a source interaction criteria is investigated with a load terminated stable regulator. Conclusions are presented in Section 6.4 .

\subsection{Load interaction analysis}

Characteristics of the payloads for switching regulators, in many practical systems, are not simple resistive loads, but are complex and frequency dependent. Including the dynamics (additional states) of the loads into the state-space expressions of the switching regulator makes analytical derivations very cumbersome. Also the results can not provide physical insight into the system and the analytical interpretation of the results may be lost. 
In order to examine the interactions between a switching regulator and its load, the performance parameters of a load terminated regulator should be derived as a function of the load terminal characteristic. Fig.6.1 illustrates the linear equivalent canonical circuit model for an unterminated switching regulator and load, $Z_{L}$. From the figure, the loop gain of the load terminated regulator, $\mathrm{T}_{G}^{*}$, can be derived as a function of the g-parameters, the loop gain of the unterminated regulator, $T_{G}$, and load impedance, $\mathrm{Z}_{\mathrm{L}}$. However, the expression for $\mathrm{T}_{\mathrm{G}}^{*}$ is still too complex to obtain any analytical insight, i.e., the stability of the regulator. Instead, as described in Section 3.2.5, Eqs.(6.1) and (6.2) provide an analytical closed form expression, from which the performance parameters of the regulator can be expressed as well defined quantities, load impedance, $Z_{L}$, and output impedance of the unterminated regulator, $g_{22}$.

$$
\begin{gathered}
\frac{v_{2}}{v_{1}}=\frac{g_{21}}{1+g_{22} / Z_{L}} \equiv \frac{g_{21}}{1+T_{L}} \\
Y_{i}=\frac{Y_{e q}}{1+g_{22} / Z_{L}}
\end{gathered}
$$

where

$$
Y_{\text {eq }}=-\left(g_{11}+\Delta G / Z_{L}\right)
$$

For the small signal analysis, the g-parameter for an unterminated regulator can easily be obtained from the known steady state output power. 


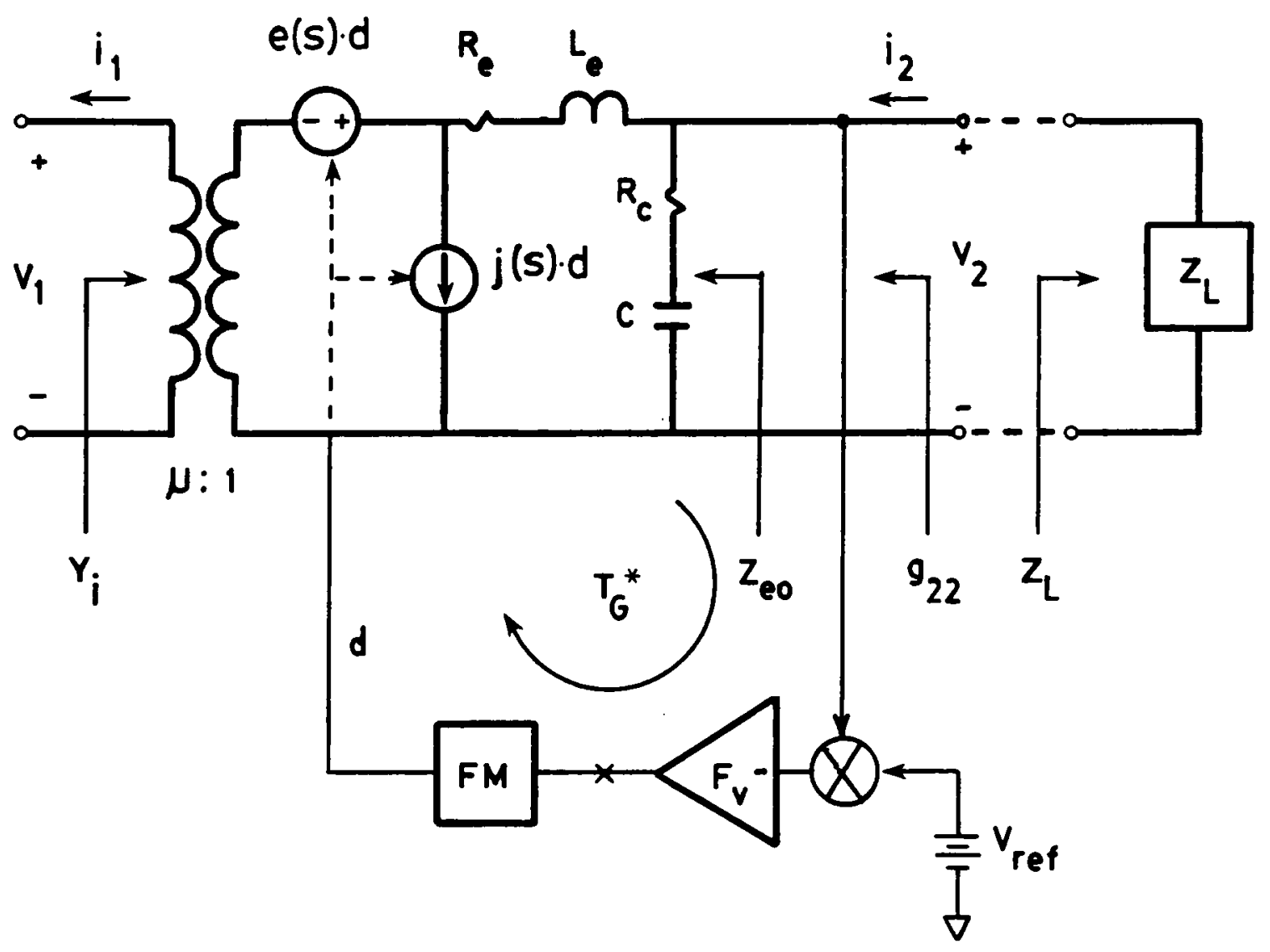

Figure 6.1 Load terminated switching regulator

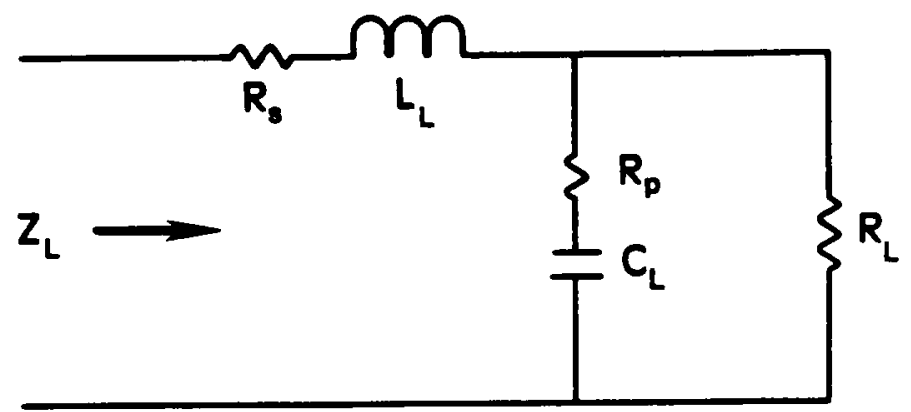

Figure 6.2 LCR load 
Thus, using the $g_{22}$ and $Z_{L}$, stability of the regulator can be determined by applying the Nyquist criterion to the quantity $T_{L}=g_{22} / Z_{L}$. Under the assumption of both $g_{22}$ and $Z_{L}$ being stable, which implies $T_{L}$ does not have RHP poles, the Bode plot technique can be used to check the stability of the regulator. Thus, from the above assumption, the sufficient condition for the stability of the regulator is simply $\left|g_{22}\right|<\left|Z_{L}\right|$.

To illustrate the load interaction problem, supposed that a payload is characterized as a simple LCR load as shown in Fig.6.2. The load impedance, $\mathrm{Z}_{\mathrm{L}}$ is then

$$
Z_{L}=\left(R_{L}+R_{s}\right) \frac{1+s /\left(Q_{L} \omega_{L}\right)+s^{2} / \omega_{L}{ }^{2}}{1+\omega_{p}}
$$

where

$$
\begin{gathered}
\omega_{L}=\frac{1}{\sqrt{L_{L} C_{L}}} \underset{\left(R_{L}+R_{p}\right)}{\left(R_{L}+R_{s}\right)}=\frac{1}{\sqrt{L_{L} C_{L}}} \text { for } R_{L} \ll R_{p}, R_{L} \ll R_{s} \\
Q_{L}=\frac{1}{\omega_{L}} \frac{R_{L}+R_{p}}{L_{L}+C_{L}\left[\left(R_{L}+R_{p}\right) R_{s}+R_{L} R_{p}\right]} \simeq \frac{1}{\omega_{L}} \cdot \frac{1}{L_{L} / R_{L}+C\left(R_{s}+R_{p}\right)} \\
\omega_{p}=\frac{1}{C\left(R_{L}+R_{p}\right) s}
\end{gathered}
$$

To illustrate this case, a single-loop (output voltage) feedback controlled buck regulator is designed. The unterminated closed-loop output 
impedance, $g_{22}$ can be directly obtained from the canonical circuit model in Fig.6.1.

$$
g_{22}=\frac{Z_{e o}}{1+T_{G}}
$$

where

$$
Z_{\text {eo }}=\left(R_{e}+s L_{e}\right) \|\left(R_{c}+\frac{1}{s C}\right) ; \text { open loop output impedance }
$$

$T_{G}=e(s) H_{e}(s) F M F_{v}(s)$; loop gain of the unterminated regulator (6.9)

$H_{e}(s)=\frac{1+s / \omega_{z}}{1+s /\left(Q_{0} w_{0}\right)+s^{2} / \omega_{0}^{2}} ;$ effective filter transfer function

$$
\omega_{0}=\frac{1}{\sqrt{L_{e} C}}, \quad Q_{0}=\sqrt{\frac{L_{e}}{C}} \frac{1}{R_{e}+R_{c}}, \quad \omega_{z}=\frac{1}{C R_{c}}
$$

for the buck regulator,

$$
L_{e}=L, \quad R_{e}=R_{\ell}, \quad e(s)=V_{2} / D
$$

Parameter values used for $Z_{L}$ are $L_{L}=5 \mu \mathrm{H}, R_{s}=5 \mathrm{~m} \Omega$ and $R_{p}=10 \mathrm{~m} \Omega$. In Fig.6.3, the locus of the critical eigenvalue as function of $\mathrm{C}_{\mathrm{L}}$ of $\mathrm{Z}_{\mathrm{L}}$ is plotted. As the value of $C_{L}$ increases, the locus approaches the imaginary axis and finally cause the regulator to become unstable. The interaction criteria can be observed from the comparison of the magnitude 
of $g_{22}$ and $Z_{L}$ as shown in Fig.6.4. For the larger value of $C_{L}$, $\omega_{p}$ in Eq.(6.6) becomes smaller and $Q_{L}$ in Eq.(6.5) becomes larger, thus more interaction occurs between $g_{22}$ and $Z_{L}$. Fig.6.5 shows the loop gain $T_{G}^{*}$ defined at Point $X$ for the corresponding values of $\mathrm{C}_{\mathrm{L}}$ in Fig.6.4. It also includes $T_{G}^{*}$ for a resistive load, $R_{L}$ (i.e. $L_{L}=0, C_{L}=0$ ). As the value of $C_{L}$ increases the bandwidth of the loop gain decreases and the phase margin also decreases, and the system becomes unstable for $C_{L}=500 \mu F$.

As commonly exercised, a filter capacitor is added between the power supply and equipment (load). This can cause system instability as illustrated in the previous example. Equipment that require a more complicated input filter create a larger degree of uncertainty of the system stability. Also, the load interaction problem is more critical when a regulator supplies the power for equipment that requires a low voltage and a high current. Two-port modeling allows one to trouble-shoot the system and to obtain design guide lines of the input filter for the equipment. 
143

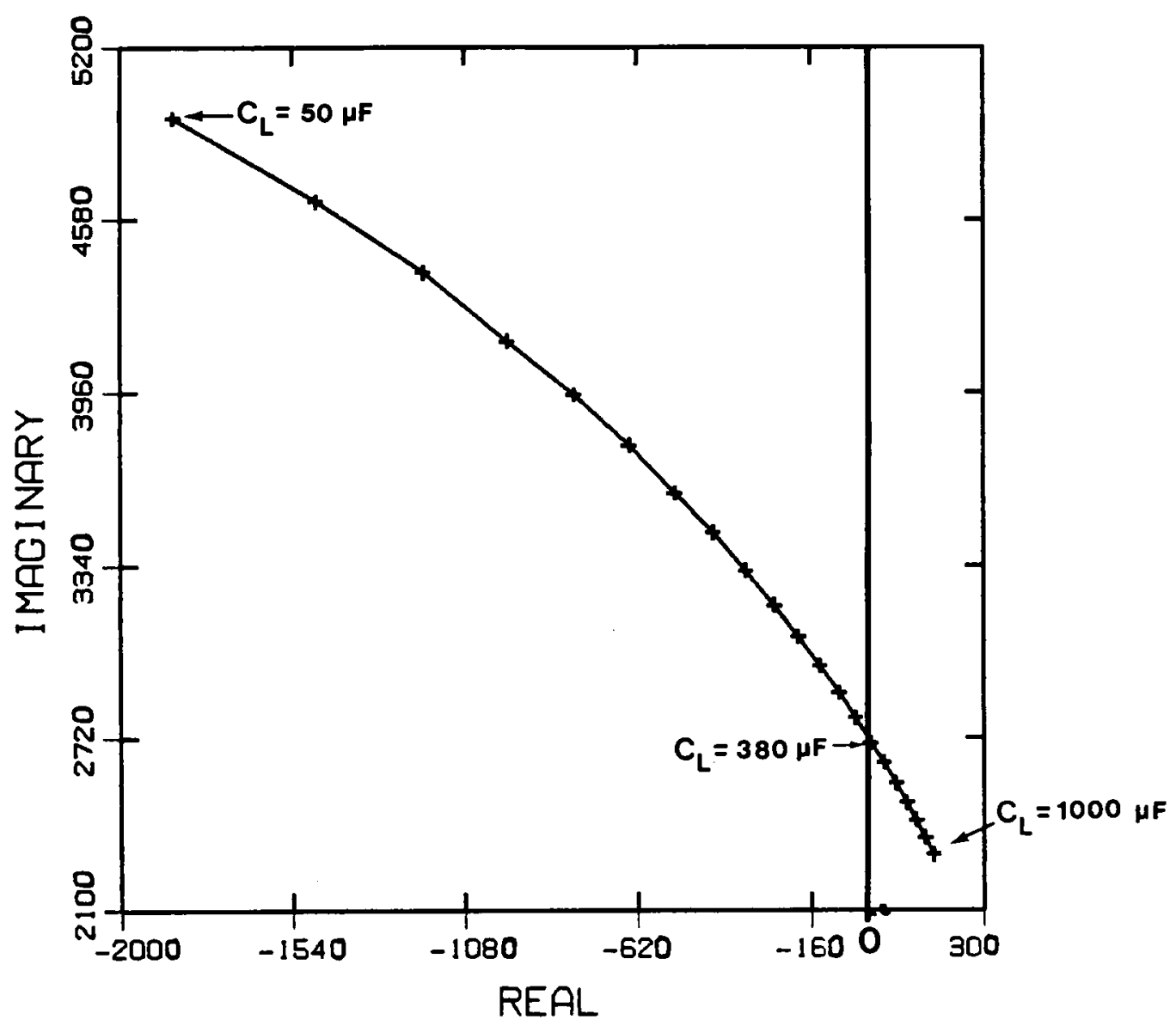

Figure 6.3 Locus of the critical eigenvalue as a function of the load capacitance $C_{L}$ 


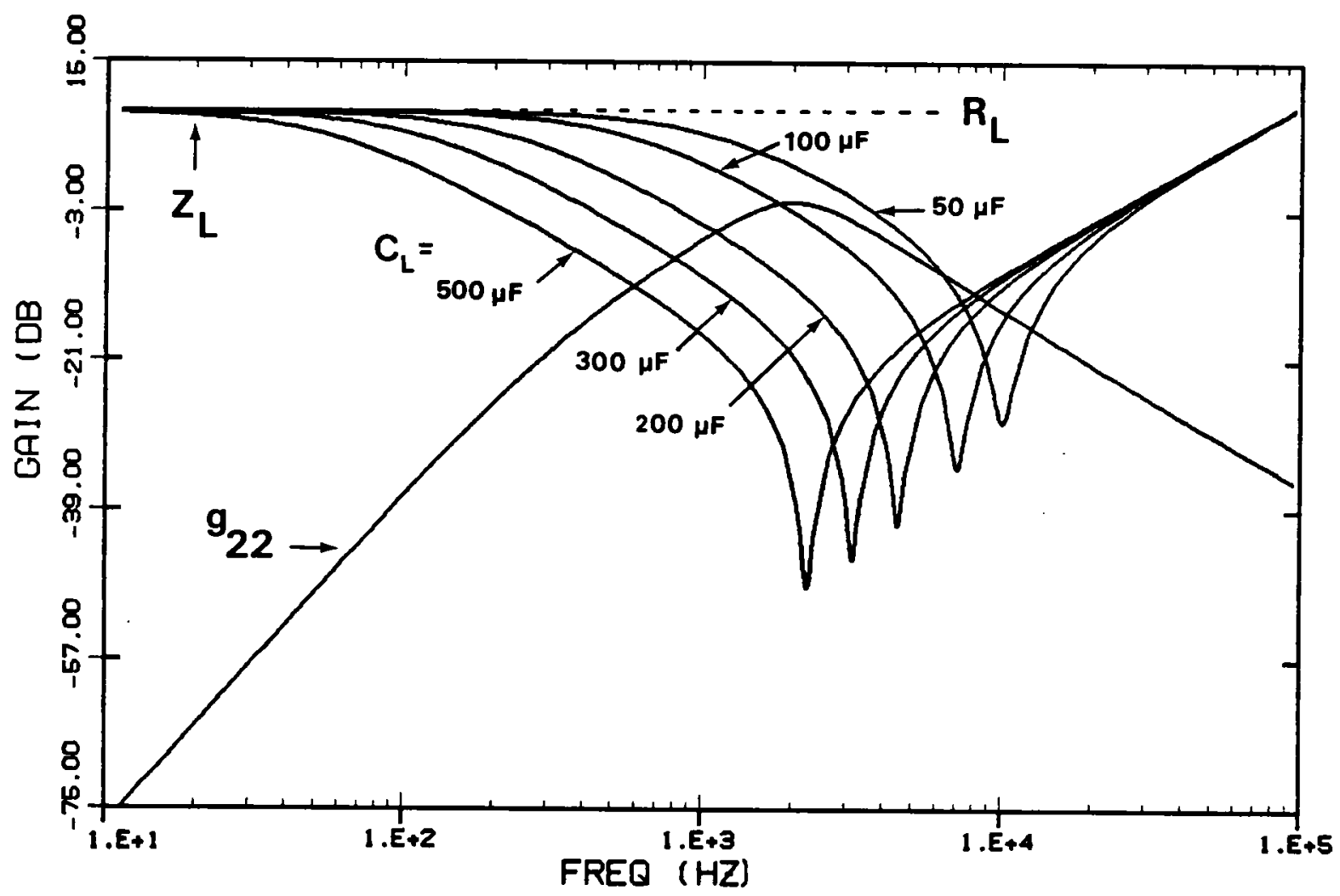

Figure 6.4 Magnitude of the impedance comparisons, $g_{22}$ vs $Z_{L}$ for various values of $C_{L}$ 


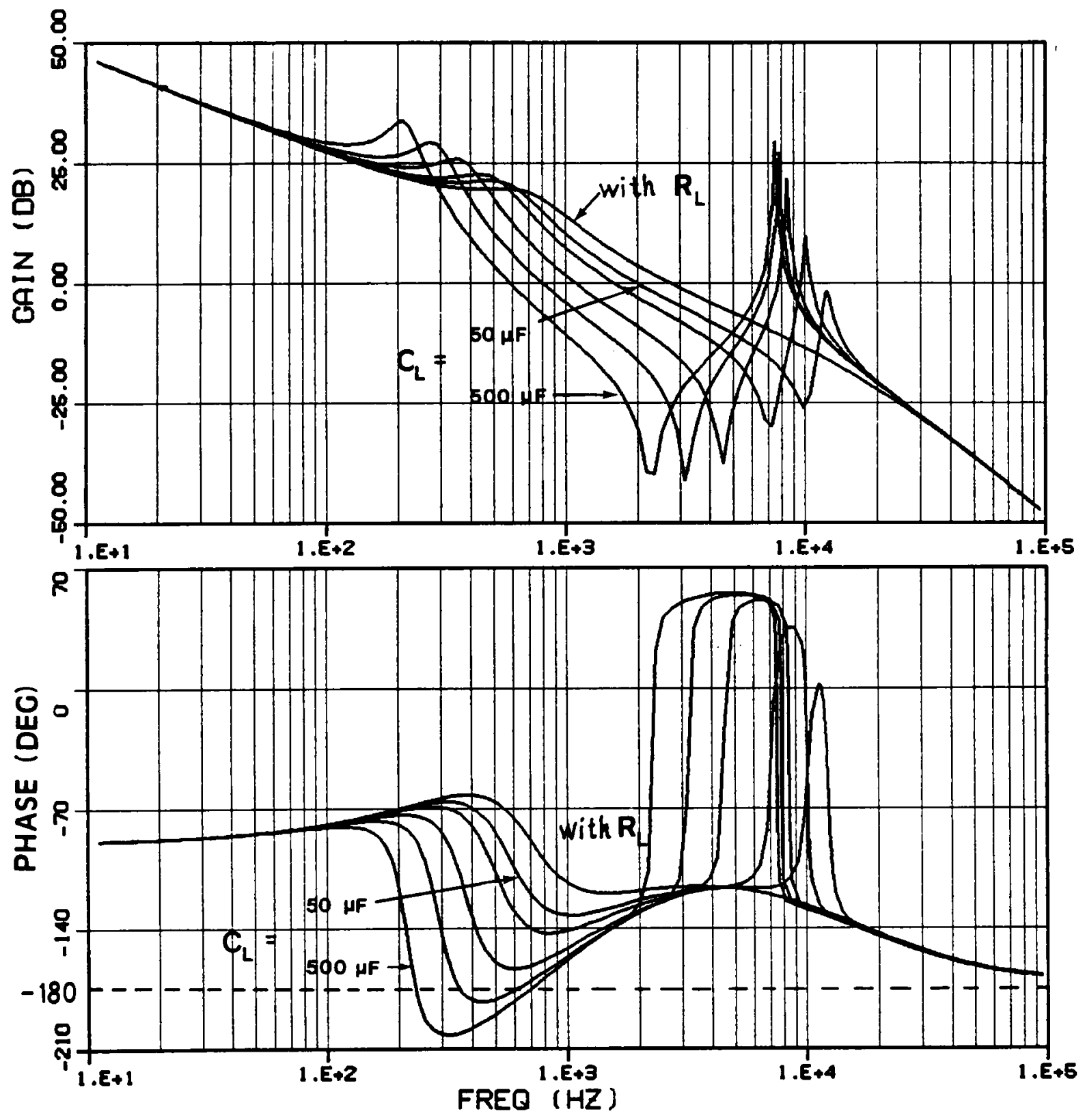

Figure 6.5 Loop gain $\left(T_{C}^{*}\right)$ characteristics for the corresponding values of $C_{L}$ in Fig.6.4 


\subsection{Source interaction analysis}

The source interaction problem of switching regulators was first described between an input filter and a regulator [13]. The conclusion can easily be extended to a general cascade configuration where a preceding block is viewed as a source impedance to the following block which is a switching regulator. This interaction analysis can be further extended to a parallel configuration as in the DET system. Fig.6.6 shows the system in which switching regulators are sharing a common bus. The source block represents the solar array, the shunt regulator or the battery discharger, cable impedances and filters.

As described in the previous section, it is very difficult to analytically characterize the performance parameters of the regulator including dynamics of the source. However, using Eq.(6.13), the source interaction can easily be analyzed similar to the load interaction analysis.

$$
H_{s}=\frac{v_{1}}{v_{s}}=\frac{1 / Y_{i}}{1 / Y_{i}+Z_{s}}=\frac{1}{1+Z_{s} \cdot Y_{i}} \equiv \frac{1}{1+T_{S}}
$$

In this case, the parameters to be used for the analysis are the input impedance of the load terminated regulator, $Z_{i}$, and the source impedance seen by the regulator, $Z_{s}$.

In the parallel configuration shown in Fig.6.6, one can define two types of interactions: local and global. The local interaction is the interaction between the source impedance seen by $j^{\text {th }}$ regulator, $z_{s j}$ and 


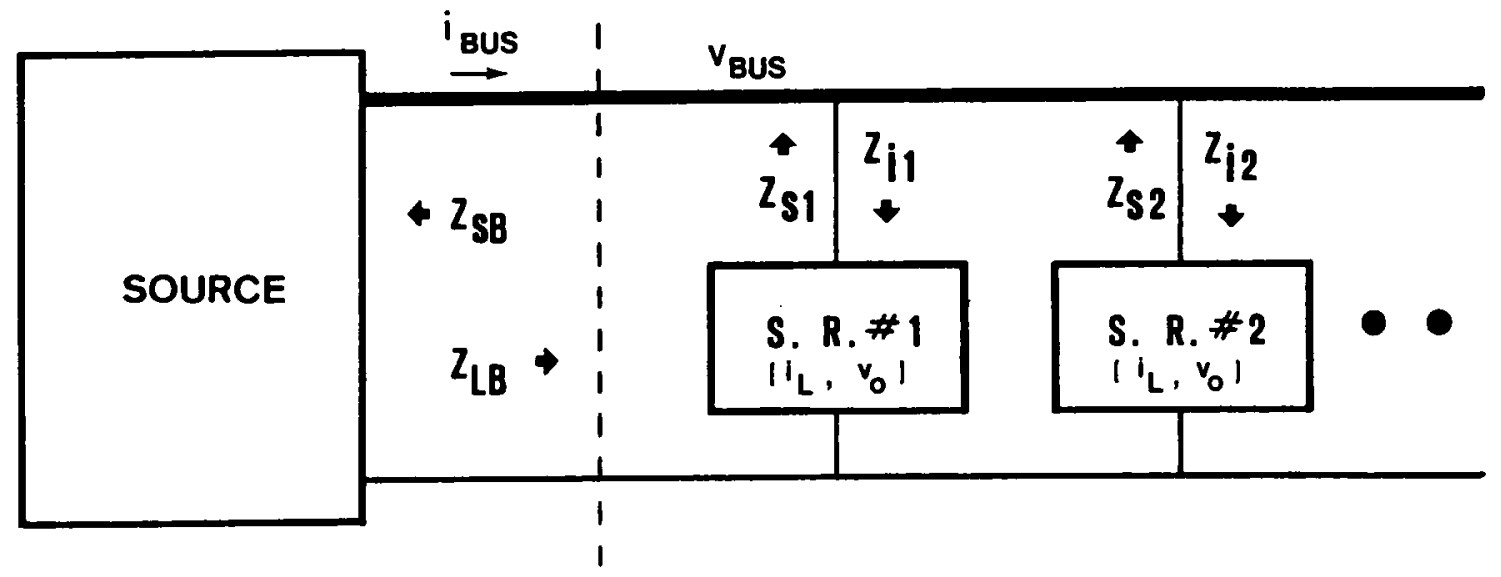

Figure 6.6 Parallel connection of switching regulators 
its input impedance, $\mathrm{Z}_{i j}$. The global interaction is the interaction between the source and load impedances seen by the main bus, $Z_{S B}$ and $Z_{L B}$. For the source interaction, let us assume that each interacting quantity $\left(Z_{s j}, Z_{i j}, Z_{S B}, Z_{L B}\right)$ is individually stable, otherwise the subsystem(s) is(are) already unstable before we consider this interaction problem. From Eq.(6.13), the system is stable if the polar plot of $T_{S}$ does not encircle $(-1,0)$ point under the above assumptions. Thus, the condition $\left|\mathrm{T}_{\mathrm{S}}\right|<1$ ensures the above stability criterion. As the number of regulators increases the stability condition moves in the worse direction and may cause instability of the system because of the negative input impedance characteristic of the regulators.

Let us consider first the undesired interaction that occurs to a local $j^{\text {th }}$ switching regulator. The source impedance seen by the $j^{\text {th }}$ regulator, $Z_{s j}$ is the parallel combination of the output impedance of the source block, $Z_{S B}$, and the input impedance of all other switching regulators, $\mathrm{Z}_{\mathrm{Lj}}$, as described in Eqs. (6.14) and (6.15).

$$
\begin{gathered}
Y_{s j}=Y_{S}+Y_{L j}, Z_{s j}=1 / Y_{s j} \\
Y_{L j}=\sum_{\substack{k=1 \\
k \neq j}}^{n} Y_{i k}
\end{gathered}
$$

To see how other regulators might affect the stability of the $j^{\text {th }}$ regulator, let us examine the summing effect of these impedances at low frequencies. Since, at low frequencies, switching regulators have the 
negative input impedance characteristic, so as $\mathrm{Z}_{\mathrm{Lj}}$, and the magnitude of $\mathrm{Z}_{\mathrm{Lj}}$ is supposed to be larger than that of $Z_{S B}$ for a stable design, the magnitude of the equivalent source impedance seen by the $j^{\text {th }}$ regulator, $\left|Z_{s j}\right|$ decreases as the number of regulators increases. This shows that a stable system can become unstable if more switching regulators are added to the main bus.

An undesired interaction in the global sense can occur when a large number of converters share the same DC bus which is not a stiff voltage source. In this case the magnitude of the load impedance seen by the bus $\left|Z_{L B}\right|$, which again is negative at low frequency, decreases as the number of regulators increases as shown in Eq. (6.16).

$$
Y_{L B}=\sum_{k=1}^{n} Y_{i k}, \quad Z_{L B}=1 / Y_{L B}
$$

When the $\left|Z_{L B}\right|$ is of the same order of magnitude as the $\left|Z_{S B}\right|$ an undesired interaction can occur. In this case the entire bus may oscillate. In this case, the local interaction may not be directly observable from the impedance comparison between $Z_{s j}$ and $Z_{i j}$, because $Z_{s j}$ may be already unstable due to the global interaction thus it may have RHP poles.

The following cases are generated to illustrate the undesired interaction problems described above.

First, a stable system in Fig.6.6 is constructed with only one switching regulator (S.R.非 - Buck regulator with input filter). Fig.6.7 shows the step load responses where the load power of S.R. 非 1 steps up from 

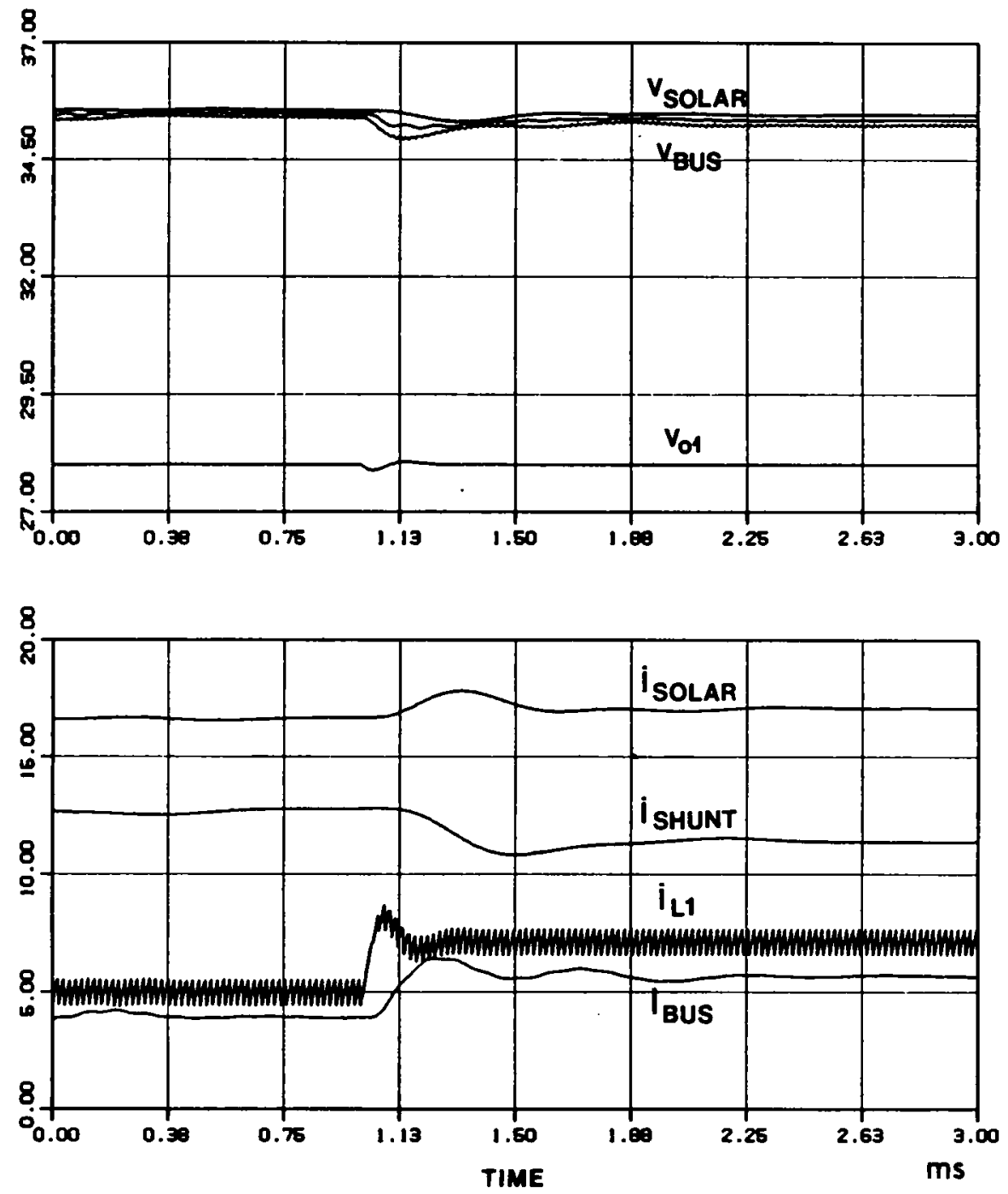

Figure 6.7 Step transient responses for the DET system with one switching regulator (S.R.\#1) 
140 to 200 watts at $1 \mathrm{~ms}$. The system eigenvalues calculated under the steady-state condition are listed in Table 6.1. The results of both small signal analysis and time domain simulation show that the system is locally and globally stable.

Next, a stable switching regulator (S.R.非 - same structure as that of S.R.非 except with different parameter values) is designed. The loop gain and step load transient responses of S.R.非 with an ideal source are shown in Fig.6.8.

Now, the S.R.非 is connected to the bus, in parallel with S.R. 非, and the transient analysis of the first case (Fig.6.7a) is repeated. Fig.6.9a shows that the new system is very oscillatory. The system eigenvalues as shown in Table 6.2 indicate a lower frequency oscillation of the bus voltage. Similarly, by comparing the input impedance $Z_{i 2}$ of S.R. 非 and the source impedance $Z_{s 2}$ seen by $S . R . k_{2}$ as shown in Fig.6.9b, the oscillatory nature of the system can be predicted. Since the two impedance curves are overlapping in Fig.6.9b, the stability predicted with the individual regulator in Fig.6.8b is no longer valid because of a parallel interaction.

To illustrate the undesired interactions in the global sense, let us consider four well-behaved switching regulators sharing a common bus. In this case identical switching regulators are used. The system is stable with a single regulator as Table $6.3 a$ indicates. However, the system becomes unstable with four regulators in parallel as shown in Table 6.3b. Fig.6.10 compares the source impedance, $\mathrm{Z}_{\mathrm{SB}}$, and the total load impedance, $Z_{L B}$, seen by the bus and reveals the undesired interactions. 
(a)
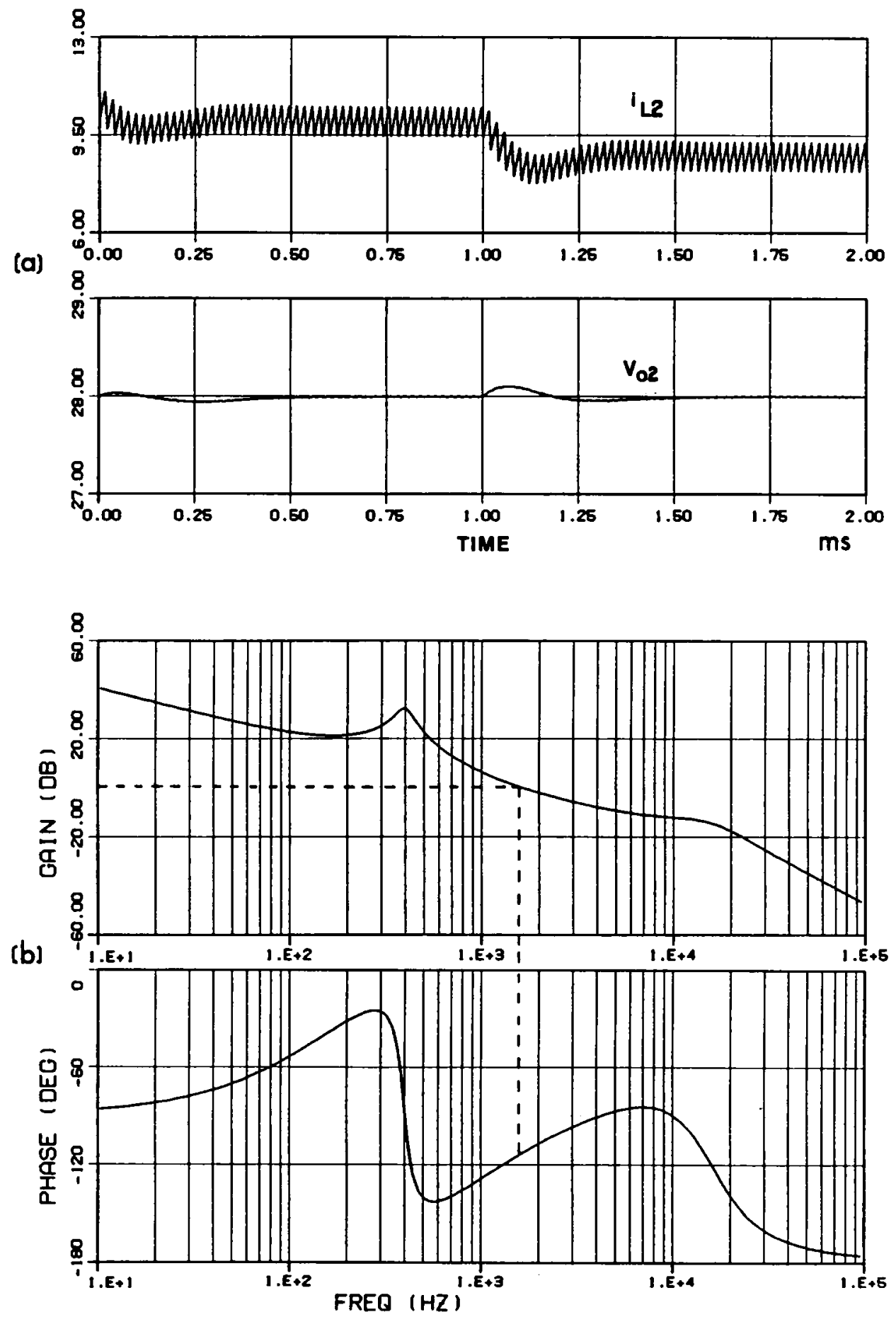

Figure 6.8 A switching regulator (S.R.\#2) responses before it is connected to the system in Fig.6.7

(a) Step load transient response -

load step : 280 to 224 watt at $1 \mathrm{~ms}$

(b) Loop gain characteristic 

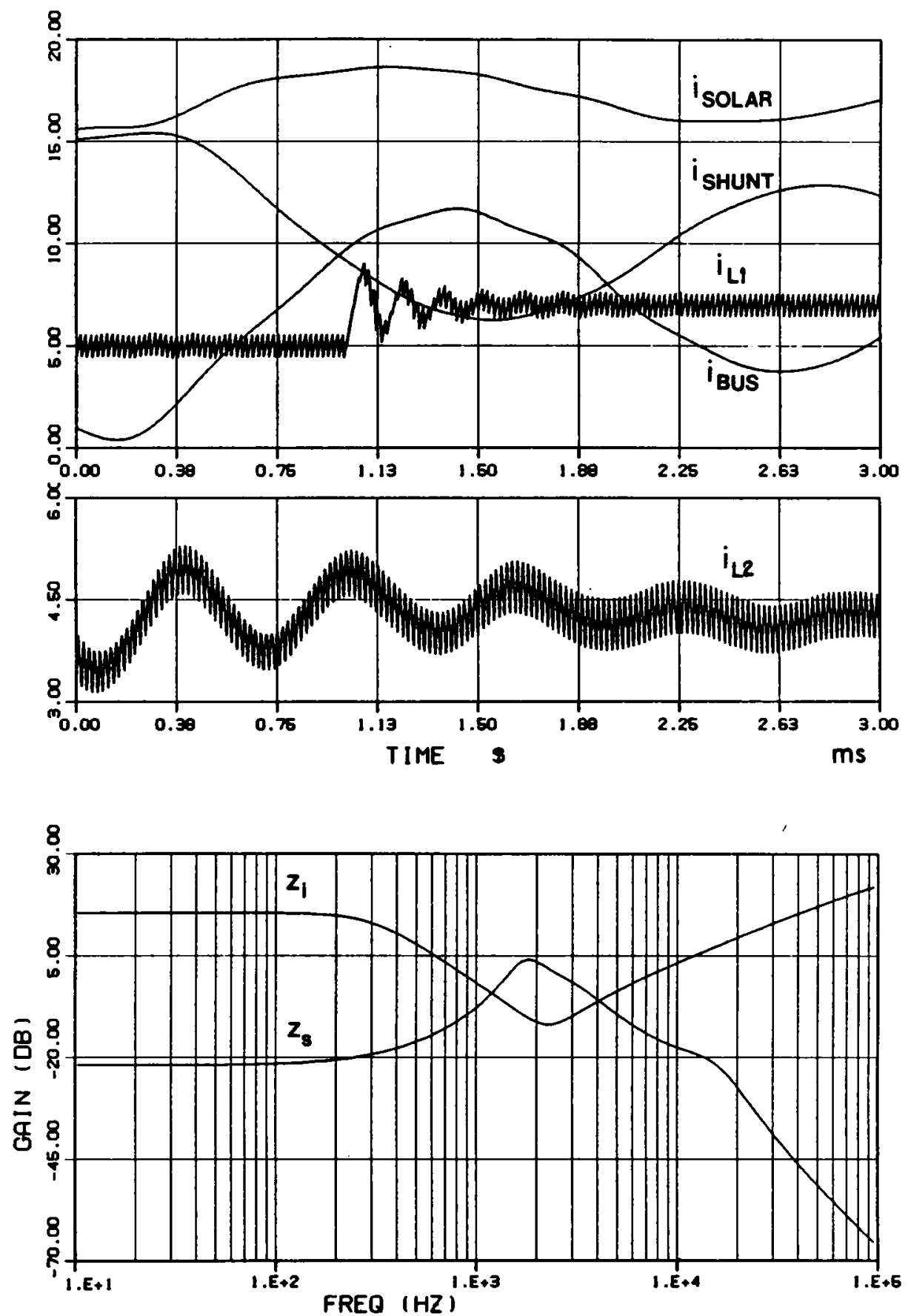

Figure 6.9 The system responses after the stable regulator (Fig.6.8) is connected to the stable system (Fig.6.7)

(a) Step transient responses -

the same step load for S.R.\#1 as in Fig.6.7

(b) The magnitude of the impedances, $\left|z_{s 2}\right| v s\left|z_{i 2}\right|$ 
15 EIGENVALUES

$\begin{array}{lc}\text { REAL } & \text { IMAGINARY } \\ -692.671 & +-489.278 \\ -708.619 & 0.000000 \mathrm{C}+00 \\ -784.905 & 0.000000 \mathrm{E}+00 \\ -4046.70 & +-4464.83 \\ -10033.1 & 0.000000 \mathrm{~B}+00 \\ -13675.9 & +-112464 . \\ -14876.1 & 0.000000 \mathrm{E}+00 \\ -500394 . & +-0.316578 \mathrm{E}+08 \\ -522133 . & +-660439 \\ -.10268 \mathrm{i}+07 & 0.000000 \mathrm{E}+00\end{array}$

Table 6.1 The eigenvalues of the system for the responses in Fig.6.7

21 EIGENVALUES

\begin{tabular}{lr} 
REAL & \multicolumn{1}{c}{ IMAGINARY } \\
--87.937 & +-2514.21 \\
-592.471 & $0.000000 E+00$ \\
-808.237 & $0.000000 E+00$ \\
-971.228 & $0.000000 E+00$ \\
-4047.86 & +-4465.36 \\
-6164.15 & $0.000000 E+00$ \\
-10033.1 & $00000000 E+00$ \\
-12460.7 & +-110556 \\
-14876.1 & $0.000000 E+00$ \\
-22909.5 & $0.000000 E+00$ \\
-157168. & $0.000000 E+00$ \\
-250118. & $+-0.223800 E+08$ \\
-500391. & $+-0.316579 E+08$ \\
-522131. & +-660434 \\
$-.102681 E+07$ & $0.000000 E+00$
\end{tabular}

Table 6.2 The eigenvalues of the system for the responses in Fig.6.9 


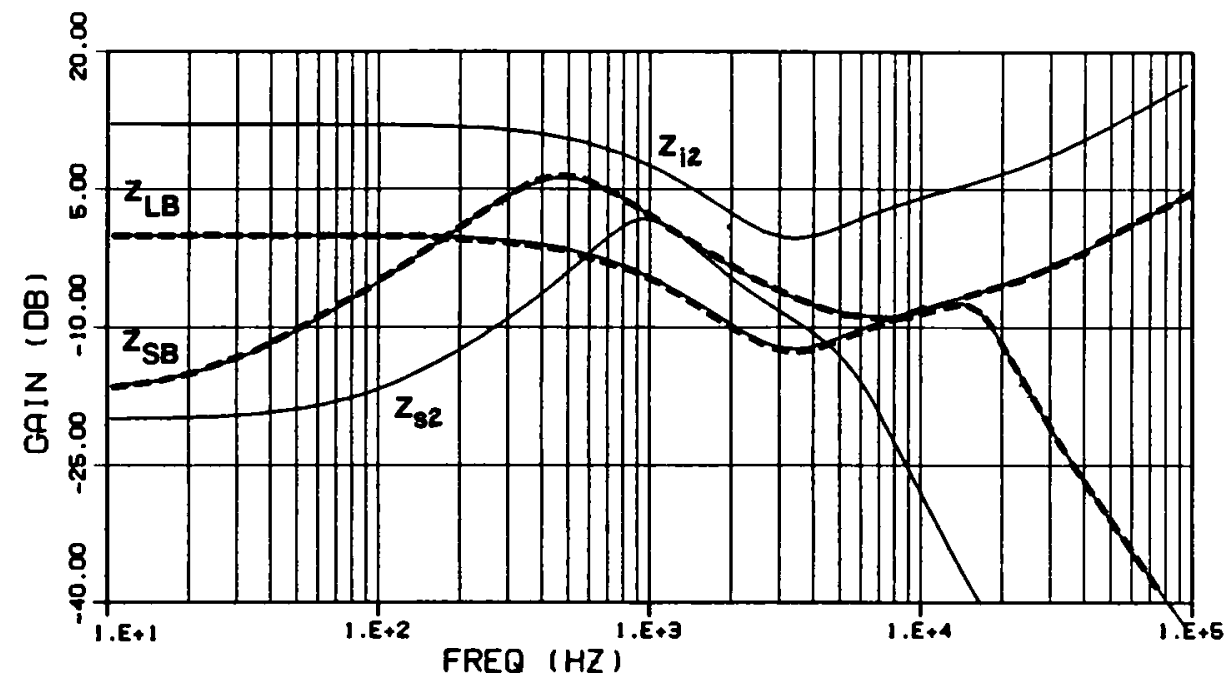

Figure 6.10 The magnitude of the impedances seen by the bus $\left(\left|z_{S B}\right| v s\left|z_{L B}\right|\right)$ and seen by S.R.\#2 $\left(\left|z_{s 2}\right| v s\left|z_{i 2}\right|\right)$

15 EIGENVALUES

$\begin{array}{lc}\text { REAL } & \text { IMAGINARY } \\ -670.571 & +-127.77 \\ -705.940 & 0.000000 \mathrm{E}+00 \\ -982.262 & 0.000000 \mathrm{E}+00 \\ -3447.72 & +-3670.94 \\ -7411.92 & +-12070.5 \\ -114902 . & 0.000000 \mathrm{E}+00 \\ -233717 . & 0.000000 \mathrm{E}+00 \\ -271348 . & +-184089 . \\ -907316 . & 0.000000 \mathrm{E}+00 \\ -.249996 \mathrm{E}+08 & +-0.999745 \mathrm{E}+09\end{array}$

33 EIGENVALUES

$\begin{array}{lc}\text { REAL } & \text { IMAGINARY } \\ -168.2244 & +-909.417 \\ -342.149 & 0.000000 \mathrm{E}+00 \\ -855.722 & 0.000000 \mathrm{E}+00 \\ -855.722 & 0.000000 \mathrm{E}+00 \\ -855.722 & 0.000000 \mathrm{E}+00 \\ -1218.23 & 0.000000 \mathrm{E}+00 \\ -3463.95 & +-3681.63 \\ -4083.17 & +-7396.58 \\ -4083.17 & +-7396.58 \\ -4083.17 & +-7396.58 \\ -17934.0 & +-16539.5 \\ -114106 . & 0.000000 \mathrm{E}+00 \\ -114106 . & 0.000000 \mathrm{E}+00 \\ -114106 . & 0.000000 \mathrm{E}+00 \\ -118324 . & 0.000000 \mathrm{E}+00 \\ -233717 . & 0.000000 \mathrm{E}+00 \\ -259219 . & +-179965 . \\ -907316 . & 0.000000 \mathrm{E}+00 \\ -.249996 \mathrm{E}+08 & +-0.999695 \mathrm{E}+09 \\ -.249996 \mathrm{E}+08 & +-0.999695 \mathrm{E}+09 \\ -.249996 \mathrm{E}+08 & +-0.999695 \mathrm{E}+09 \\ -.249996 \mathrm{E}+08 & +-0.999895 \mathrm{E}+(19\end{array}$

Table 6.3 Eigenvalues of the system

(a) with single switching regulator (b) with four switching regulators 
However, by comparing the source impedance, $Z_{s 1}$, and the input impedance, $Z_{i 1}$, seen by the individual regulators, the presence of the undesired interaction to an individual regulator is not obvious. From Eq.(6.13), the sufficient condition for stability is $\left|z_{i}\right|>\left|z_{s}\right|$ under the assumption that both $Z_{i}$ and $Z_{s}$ are stable. In this case, the source impedance, $Z_{s}$, seen by S.R.झ⿰1 is unstable (has RHP poles) because the undesired interaction already occured between $Z_{S B}$ and $Z_{L 1}$. Thus, even though $\left|Z_{i 1}\right|>$ $\left|Z_{s j}\right|$ for all frequencies as shown in Fig.6.10, the system is unstable. This reveals that the analysis must be done at both the local regulator level and the system level.

\subsection{Conclusions}

The subsystem interaction criteria are investigated at component, subsystem, and system levels by comparing the impedances at an interacting point.

Both the load and the source interaction problems are illustrated using the switching regulator examples. The performance of the regulator depends upon the dynamic characteristics of the payload. As demonstrated in this chapter, a single filter capacitor between the regulator and equipment (load) can cause system instability due to the load interaction. The load interactions can be investigated using the output impedance of the unterminated two-port subsystem (component), $g_{22}$, and its load impedance, $\mathrm{Z}_{\mathrm{L}}$. 
For a spacecraft power system that uses many switching regulators in parallel on the bus, such as the DET system, the interaction problem should be examined both at the local regulator level and the system level. Also, the analysis shows that as the number of regulators increases, the margin of stability of the system tends to diminish due to the parallel interactions. 


\section{Chapter 7}

\section{EMPIRICAL DATA MODELING AND ANALYSIS}

\subsection{Introduction}

As discussed in Chapter 2, defining mathematical models through experimental testing of an actual system (or scaled model) is, generally, least intensive in modeling effort and the empirical data model is accurate in predicting the overall system behavior. In general, experimental data serves two major functions: model verification and model development. If a theoretical model has been developed, at some point its validity must be checked with actual experimental test data to have a high level of confidence in its predictive capabilities. Also, some systems defy refined theoretical treatment due to the lack of an assessment of certain parameter values, thus, experimentation is the only effective means of obtaining models with acceptable accuracy. In this chapter, model development based on empirical data is discussed and extended for the reduced order model realizations of a complex system.

In Section 7.2 , model development from the frequency response data and its applications are described. Algorithms for identification of model parameters identification are reviewed in Section 7.3. In Section 7.4, model development and analysis using the DET system are presented. Conclusions are presented in Section 7.5. 


\subsection{Model development from frequency response data}

Typical methods for linear system identification using empirical data are based on frequency, step, and impulse responses. Most of these methods apply to a linear process, but they may also be applicable to the linearized form of nonlinear systems if the input disturbance levels are kept low. Among these, frequency-response identification provides the most accurate model realization for the system stability. Since generation of the sinusoidal input signal and measurement of its responses, i.e. audio susceptibility, feedback loop gain, impedances, etc., are not foreign to power system design engineers, the frequency response technique is more attractive than others.

Using an automatic network analyzer that includes a multi-channel bandpass tracking filter for gain and phase measurements and a built-in sinusoidal function generator synchronized with the tracking filter, accurate frequency response data of the system can easily be obtained. Where a model of an existing subsystem is to be constructed, empirical data can be obtained that characterize the input-output properties. Based on the set of empirical data a mathematical relation can be established that reproduces the same input-output behavior as that of the actual subsystem without regard to the physical structure of the subsystem.

The empirical data modeling technique can be used for the following applications. 
Model development from experimental data:

For certain components or subsystems, such as a complex payload, cable, etc., it is often quite difficult to develop an analytical model. Under such circumstance the component or subsystem is viewed as a black box. The port characteristics of the black box can be experimentally measured by exciting an appropriate terminal with a sinusoidal source. From the experimental data, in the form of the frequency response, an analytical transfer function model can be developed using the complex curve fitting technique. The required transfer function model to represent properly the input-output characteristics of a black box will be described in the following section.

\section{Reduced order model realization:}

The model development from frequency data using the complex curve fitting technique can also be applied to reduce the order of complexity of the system. The technique is particularly powerful for large scale system modeling and analysis. For a large scale power system, computer simulation of an entire system can be very time consuming and memory capacity often exceeded. It is desirable that the entire system be broken into smaller subsystems so that an available computer system and software can be adapted. Each subsystem can then be simulated by the computer, or experimentally measured to obtained the frequency response data. From either the analytical or the empirical data a reduced order model can be realized using the complex curve fitting technique. As long as a reduced order transfer function model fits the original data reasonably well the 
dynamic performances of the reduced order subsystem should match well with that of the original system. This technique allows the overall system to be realized with a reduced order model. More importantly, it aids in the trouble-shooting and design of a component or a subsystem in a large scale system. Using this technique, attention can be focused on the "troubled" equipment or subsystem in greater detail while treating the rest of the system with a reduced order model to take into account any possible interactions.

\subsection{Review of the existing complex curve fitting algorithms}

As described in the previous section, the purpose of the frequency response approach is to develop a transfer function model from the two sets of data, the amplitude ratio and the phase difference of the input and output. While methods for fitting curves to empirical data are a well known part of classical mathematics, the need to fit two curves (amplitude and phase) simultaneously over a wide range of frequencies requires a somewhat different treatment from the classical approaches. Several methods documented in the literatures are reviewed in this section.

One of the earliest methods by Levy [14] that dealt with the fundamentals of complex curve fitting is briefly reviewed here. This method requires that the measured frequency response be given in terms of its real and imaginary parts:

$$
\frac{Q_{0}}{Q_{i}}(j \omega)=F(j \omega) \equiv R(\omega)+j I(\omega)
$$


Let the fitted analytical model be

$$
\begin{aligned}
G(j \omega) & =\frac{A_{0}+A_{1}(j \omega)+A_{2}(j \omega)^{2}+\ldots}{B_{0}+B_{1}(j \omega)+B_{2}(j \omega)^{2}+\ldots} \\
& \equiv \frac{\alpha+j \omega \beta}{\sigma+j \omega \tau} \equiv \frac{N(\omega)}{D(\omega)}
\end{aligned}
$$

The error of fitting, $\varepsilon(\omega)$ is then

$$
\varepsilon(\omega)=F(j \omega)-G(j \omega)=F(j \omega)-\frac{N(\omega)}{D(\omega)}
$$

To minimize the square of the error, $\varepsilon(\omega)$ (conventional least-square method), a set of simultaneous nonlinear equations results, whose solution is difficult. Instead, a new error function is defined by multiplying $D(w)$ into $\mathrm{Eq} \cdot(7.3)$

$$
\varepsilon^{\prime}=D(w) \varepsilon(w)=D(w) F(w)-N(w) \equiv a(w)+j b(w)
$$

Then the cost function for $m+1$ points of frequency data is defined as

$$
J=\sum_{k=0}^{m}\left[a^{2}\left(w_{k}\right)+b^{2}\left(w_{k}\right)\right]
$$


To minimize the cost function $J$ with respect to the coefficient vector, $\mathrm{p}=\left[\begin{array}{llllll}\mathrm{A}_{0} & \cdots & \mathrm{A}_{\mathrm{m}} & \mathrm{B}_{0} & \cdots & \mathrm{B}_{\mathrm{m}}\end{array}\right]^{\mathrm{T}}$, set

$$
\frac{\partial J}{\partial p}=0
$$

Eq.(7.6) leads to the linear algebraic equation

$$
\lambda p=Q
$$

where

$$
\begin{gathered}
\lambda=\mathrm{nxn} \text { coefficient matrix, } \mathrm{n}=\mathrm{m}+1 \\
\mathrm{Q}=\mathrm{n} \times \mathbf{I} \text { constant vector }
\end{gathered}
$$

Finally, the solution for the model coefficient is

$$
p=\lambda^{-1} Q
$$

This algorithm is simple allowing a standard library function for the matrix inversion routine to be directly adapted. However, this technique has some serious deficiencies. If the transfer function has to be determined for frequencies extending several decades, the matrix $\lambda$ has a "rank deficiency". In other words, the elements of the matrix $\lambda$ are such that lower frequency values have very little influence. Thus, large errors can be introduced at low frequencies. If the poles in $G(j \omega)$ vary widely throughout the experimental points a good fitted model cannot be obtained. 
To overcome the deficiencies, an iterative procedure is introduced in [15] that eliminates the weighting problem in the above method. This method improves the above algorithm by modifying Eq. (7.4) such that

$$
\varepsilon_{k}^{\prime \prime}\left(\omega_{k}=\frac{\varepsilon\left(\omega_{k}\right) D\left(\omega_{k}\right)_{L}}{D\left(\omega_{k}\right)_{L-1}}=\frac{F\left(j \omega_{k}\right) D\left(\omega_{k}\right)_{L}}{D\left(\omega_{k}\right)_{L-1}}=\frac{N\left(\omega_{k}\right)_{L}}{D\left(\omega_{k}\right)_{L-1}}\right.
$$

where the subscript $L$ corresponds to the iteration number. As $D(\omega)$ is not known initially, it is assumed to be 1 . The coefficients are obtained by minimizing the sum of $\left|\varepsilon_{k}\right|^{2}$ at all the data points. This algorithm fits the data much better over a wider frequency range than the algorithm in [14] and the iterations tend to converge rapidly.

A more recent effort in [16] uses a conjugate gradient search technique [17] to minimize the nonlinear cost function

$$
\begin{aligned}
J & =\int_{0}^{\infty}\left|F(j \omega)-A(\omega) e^{j \theta(\omega)}\right|^{2} d \omega \\
& =\sum_{k=0}^{m}\left|F\left(j \omega_{k}\right)-A\left(\omega_{k}\right) e^{j \theta\left(\omega_{k}\right)}\right|^{2}\left(\omega_{k+1}-\omega_{k-1}\right)
\end{aligned}
$$

In this algorithm, the model is allowed to be characterized by a gain, real poles and zeros, and complex poles and zeros with the damping ratios. It also allows for dead time in the model, which is not possible in the previous methods [14] and [15]. A FORTRAN program listed in [16] is 
implemented in the host software for this study.

Curve fitting results, in general, are quite satisfactory as will be seen in the following section. However, the results (or the number of iterations required for the error to be in the specified bound) depend on the initial guess of the coefficients which is especially critical when one uses a wrong sign for the initial guess.

\subsection{Empirical data modeling and analysis of power systems}

As mentioned in Section 7.2 , the required model for a subsystem depends on the modeling objectives. Whether a subsystem is considered as a black box or is realized as a reduced order model, the subsystem to be modeled can be defined in the system as either an one-port or two-port model.

One-port modeling:

To analyze the behavior of a component or subsystem whose load or source characteristic is unknown or is to be realized as a reduced order model, the load or source subsystem can be defined as an one-port network. In this case, only one transfer function model (impedance) is needed to represent the subsystem. From the frequency response data of the impedance obtained from either experimental measurement or computer simulation of the circuit model, an analytical model can be developed using the complex curve fitting technique. The system performance parameters such as stability, audiosusceptibility and impedances can be analyzed using the curve fitted model. Time histories including step transient 
responses of the system can be simulated. The step transient analysis, however, is valid for a small disturbance level. The curve fitted analytical model also allows one to analyze the source and load interaction problems.

To illustrate one-port modeling, the following cases are considered using the DET system shown in Fig.4.1.

First, assume that the switching regulator subsystem, S.R.非, is a black box that can be viewed as one of the loads on the main bus line. The black box can then be characterized by its input impedance, $Z_{i 1}$, which can easily be measured experimentally. An analytical transfer function model is then developed that represents S.R.非 as shown in Fig.7.1. Curve fitted results of $Z_{i 1}$ data using the $3^{\text {rd }}$ order model is shown in Fig.7.2. Using this model, the behavior of the system (i.e., the bus voltage) due to the disturbances at the source or at the other load (S.R.非) can be examined. To show the results of one of the aforementioned analyses, Fig. 7.5 and 7.6 compare the transfer functions from the solar array output voltage, $v_{S}$, to the bus voltage, $v_{B}$, and to the output voltage of S.R.非, $\mathrm{v}_{02}$, between the system with the actual circuit model and that with the curve fitted model. As the curve fitted result in Fig. 7.2 predicted, the system responses are almost identical between the two system.

Secondly, model the subsystem of the total load on the bus line from empirical data as shown in Fig.7.3. The frequency response data of the load impedance seen by the bus, $Z_{L B}$, is obtained either by experimental measurement or by computer simulation to realize a reduced order model. The actual circuit model which is a $16^{\text {th }}$ order model and curve fitted 
results of $Z_{L B}$ data using the $4^{\text {th }}$ order model is shown in Fig.7.4. Using this model, the behavior of the bus voltage can be examined as in the previous case. Stability of the solar array equilibrium point can be analyzed in detail. Also, the performance parameters such as the loop gain characteristic of the shunt regulator can be analytically determined. Fig.7.5 also includes the frequency response from the solar array output voltage, $v_{S}$, to the bus voltage, $v_{B}$, for this case. Again the result matches extremely well with the original system.

\section{Two-port modeling:}

As mentioned in Chapter 3, modeling of the distribution cable is very difficult. To characterize such a component it is necessary to view it as an unterminated two-port network and model its terminal characteristics. Thus, in this case, four sets of frequency response data are needed to identify the four elements in the G-matrix. As described in Fig.2.2 of Chapter 2, port-1 is excited by a voltage source for $g_{11}$ and $g_{21}$, and port-2 by a current source for $g_{12}$ and $g_{22}$. Since the hybrid g-parameters can be converted to the open circuit z-parameters or short circuit y-parameters, as shown in Eq.(7.11), appropriate experimental measurements can easily be obtained.

$$
G=\left[\begin{array}{cc}
\frac{-1}{z_{11}} & \frac{z_{12}}{z_{11}} \\
\frac{z_{21}}{z_{11}} & \frac{\Delta z}{z_{11}}
\end{array}\right]=\left[\begin{array}{cc}
\frac{-\Delta Y}{y_{22}} & \frac{-y_{12}}{y_{22}} \\
\frac{-y_{21}}{y_{22}} & \frac{1}{y_{22}}
\end{array}\right]
$$




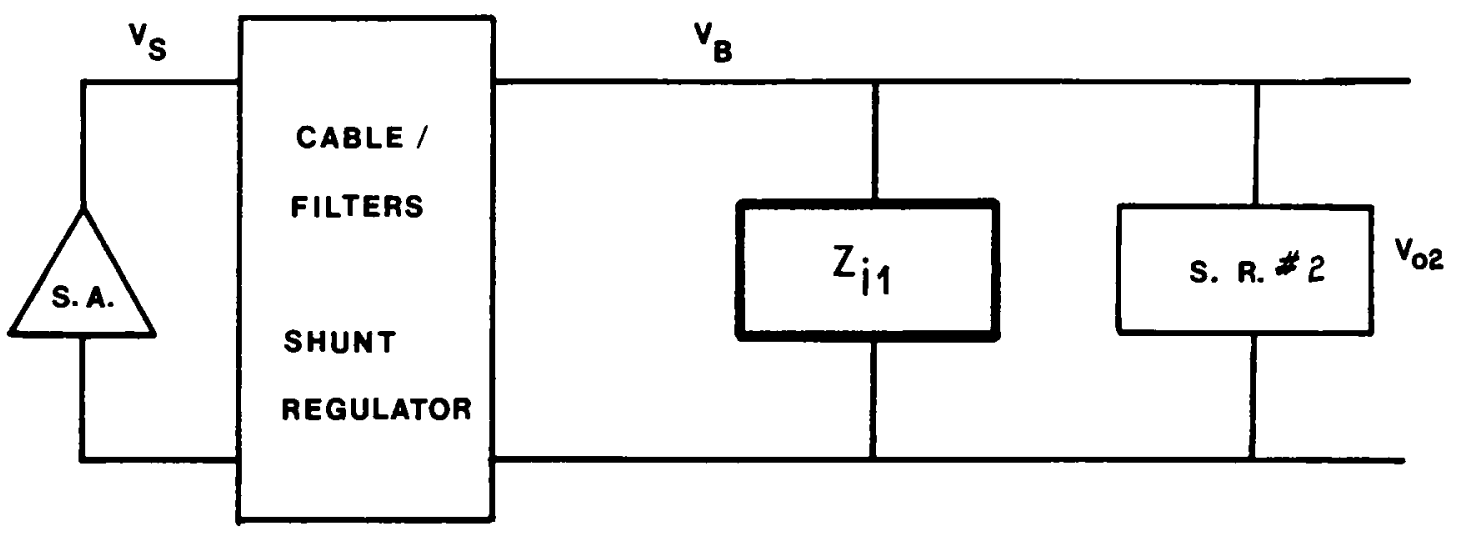

Figure 7.1 DET system with the empirical data curve fitted model for S.R.\#1
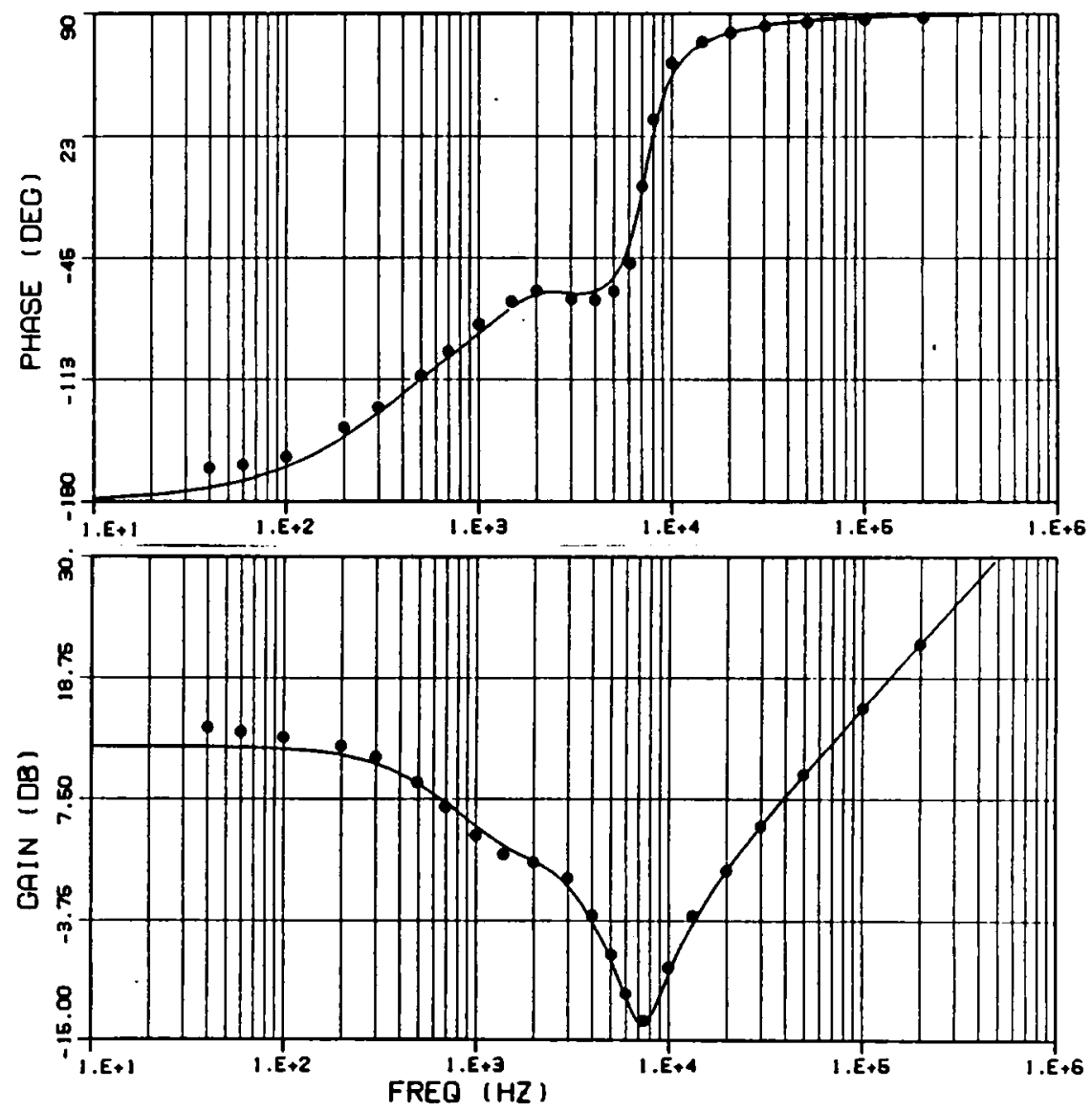

Figure 7.2 Curve fitting for $z_{i 1}$

... : empirical data

: curve fitted analytical model 


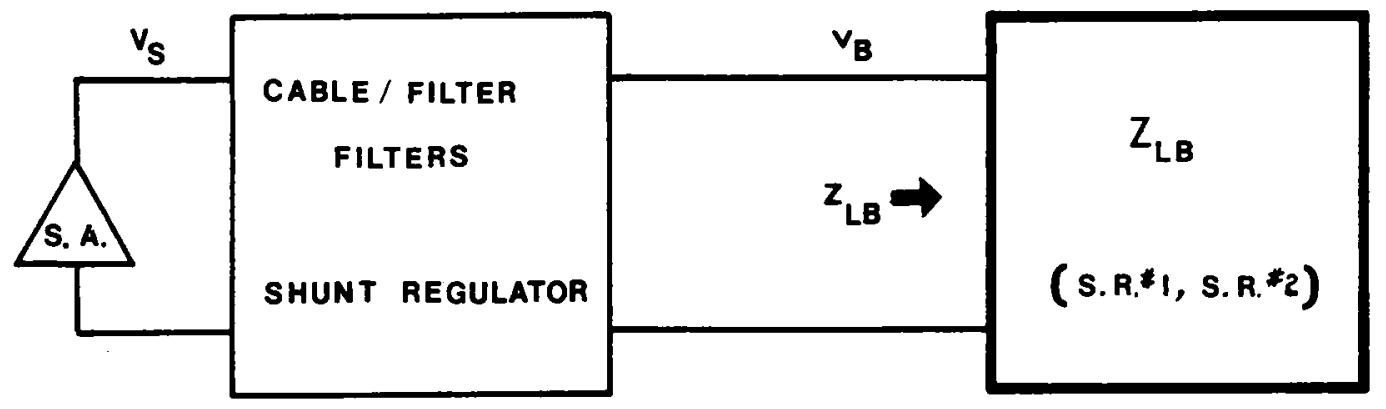

Figure 7.3 DET system with the reduced order model ( $z_{L B}$ for S.R.\#1 and S.R.\#2)

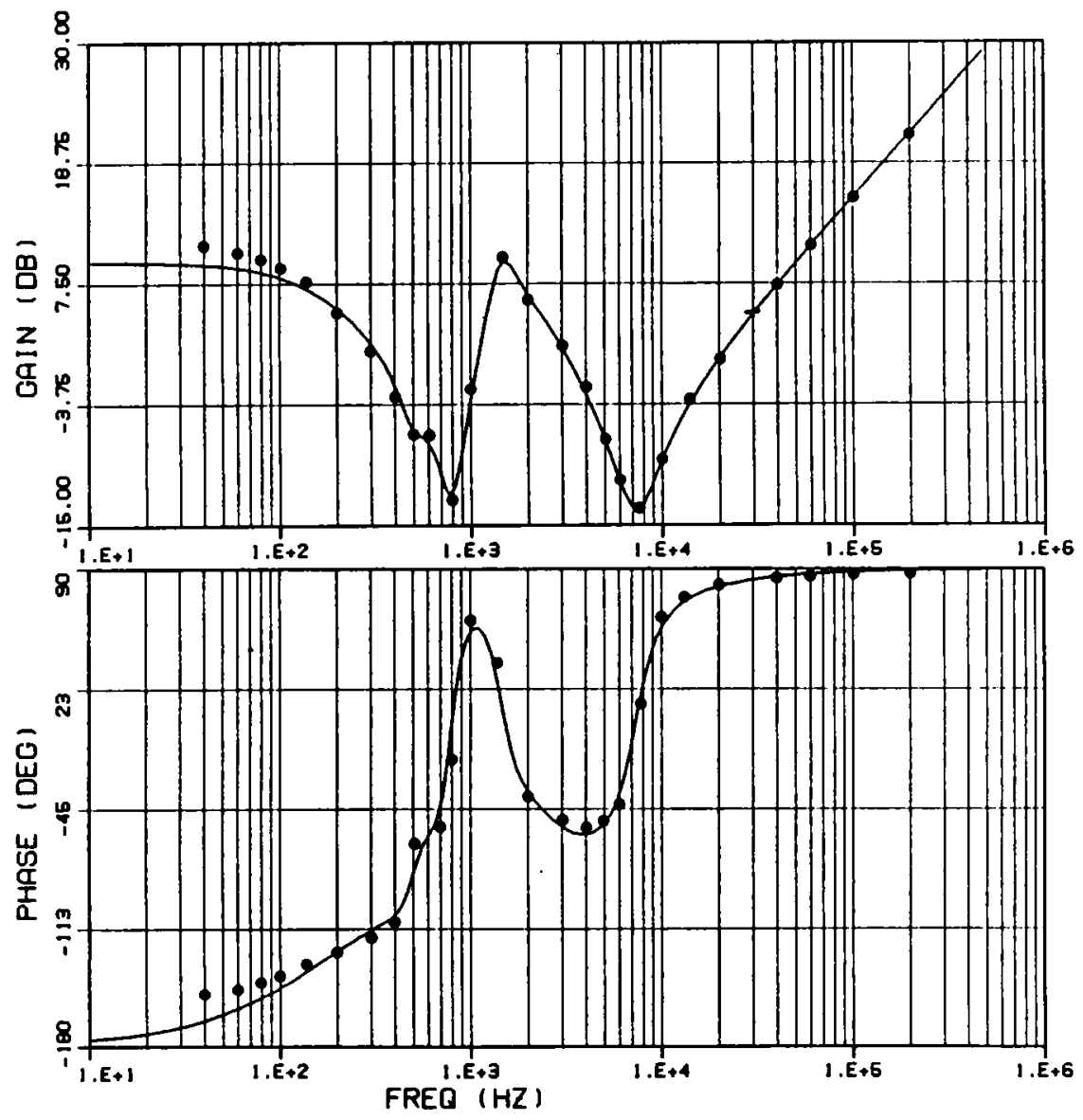

Figure 7.4 Curve fitting for $z_{L B}$

.... empirical data of the $16^{\text {th }}$ order circuit model

- curve fitted $4^{\text {th }}$ order analytical model 


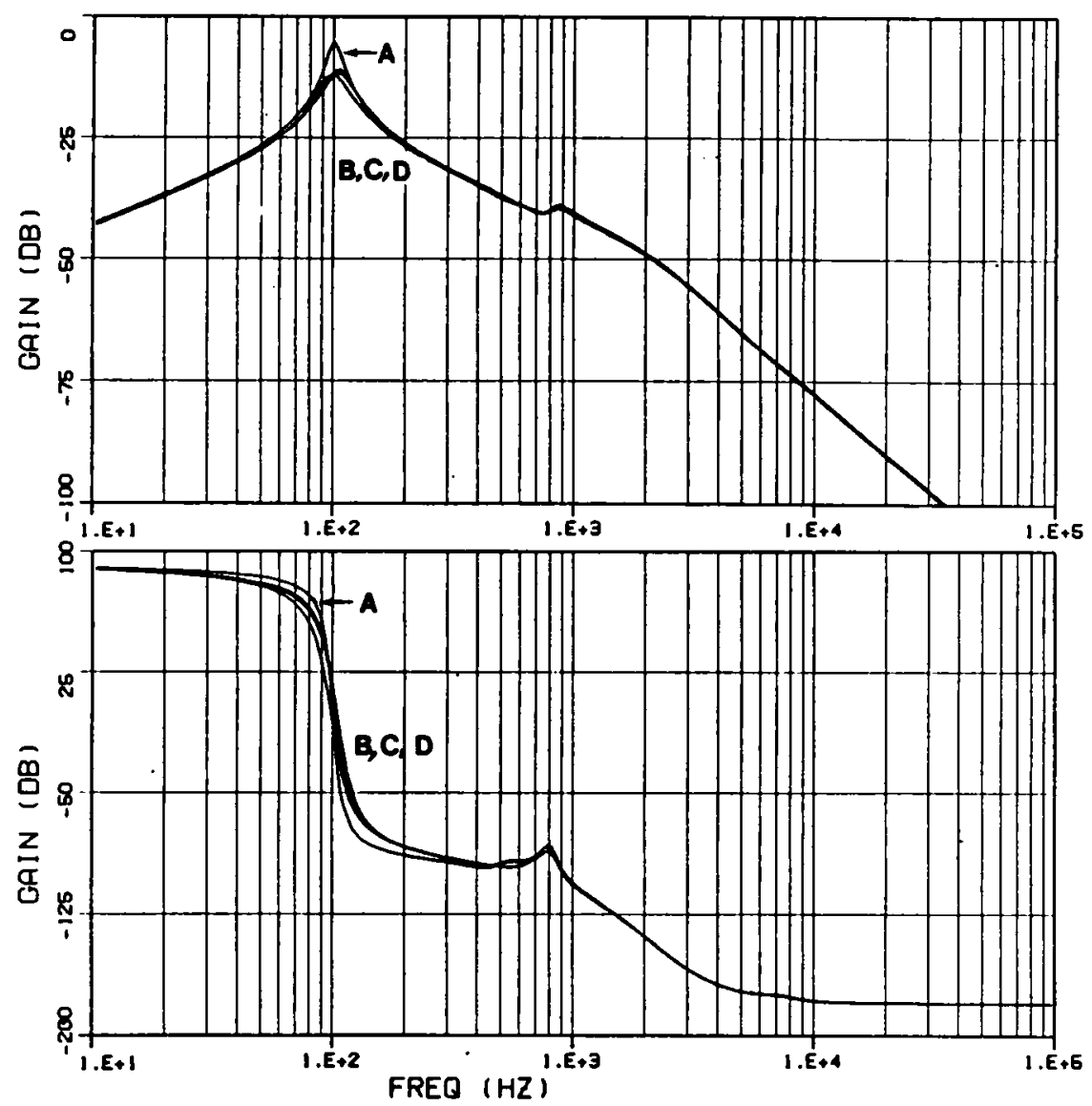

Figure 7.5 Frequency response from $v_{s}$ to $v_{B}$
A : The exact system model
$B$ : with empirical data model for $z_{i 1}$ in Fig.7.1
$C$ : with reduced order model for $z_{L B}$ in Fig.7.3
$D$ : with two-port fitted model for the cable in Fig.7.7 

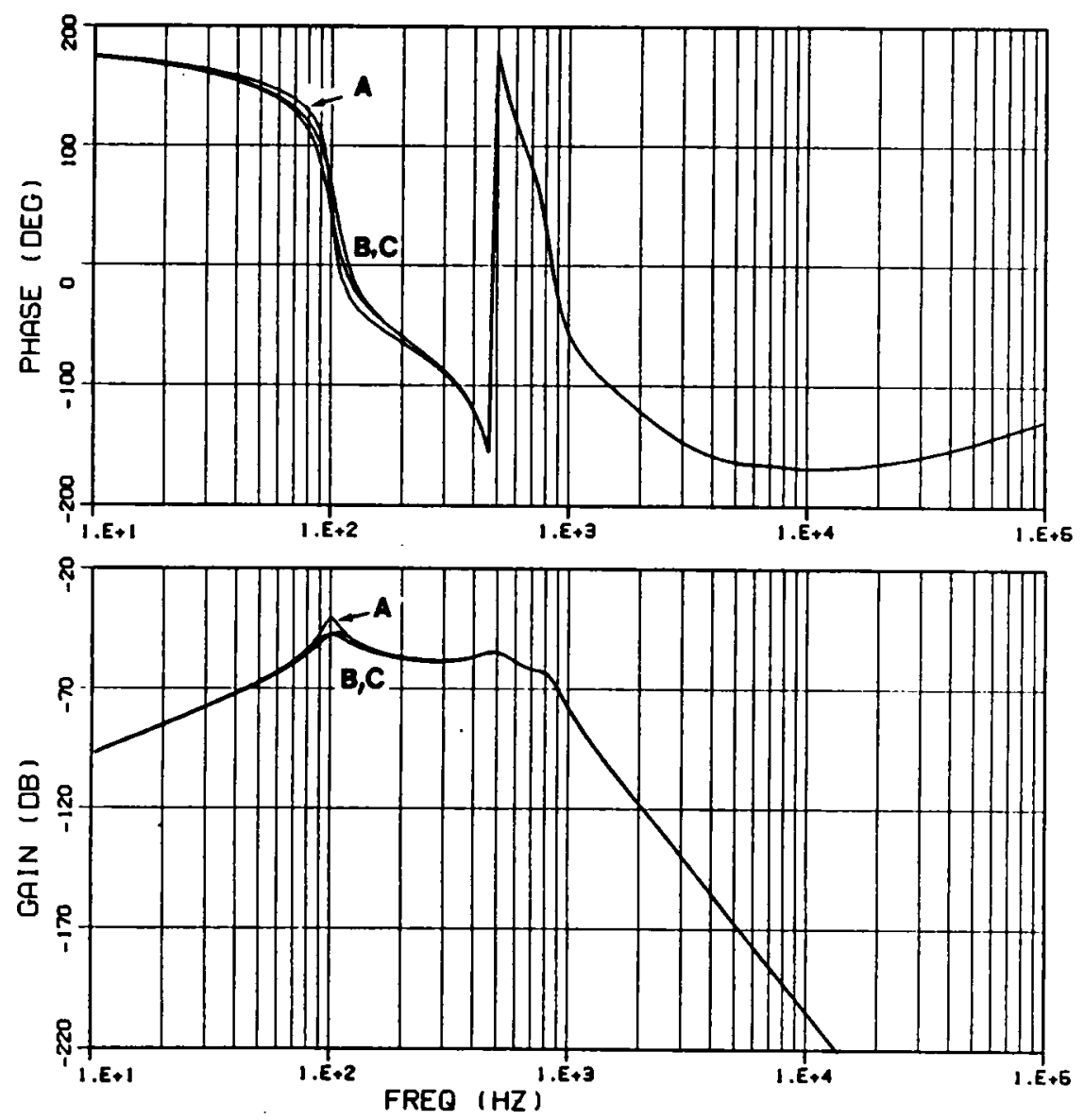

Figure 7.6 Frequency response from $v_{s}$ to $v_{02}$

$A$ : The exact system model

$B$ : with empirical data model for $z_{i 1}$ in Fig.7.1

$D$ : with two-port fitted model for the cable in Fig.7.7 


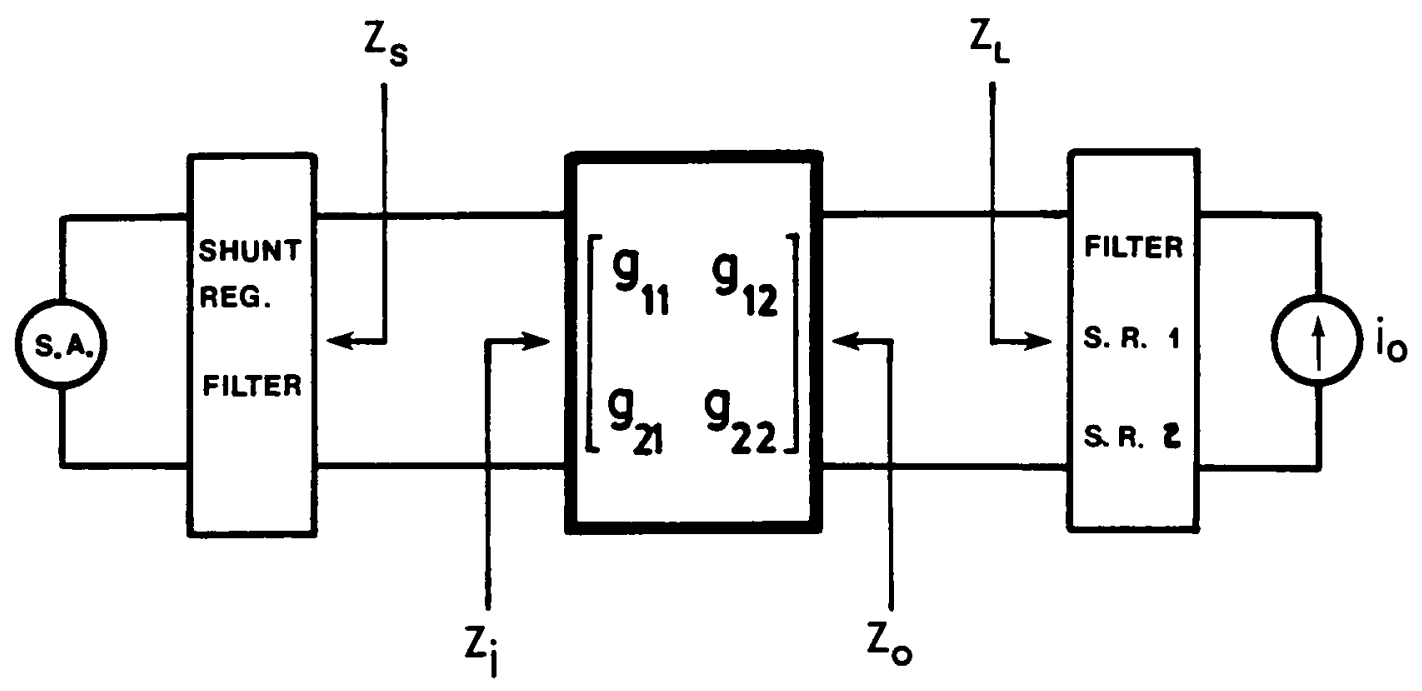

Figure 7.7 Empirical data modeling of two-port component (Cable) 
The G-matrix model fully characterizes the two-port black box. The model is connected to the system according to the interconnection law which results in a doubly terminated two-port system.

As shown in Fig.7.7, the two-port cable/filter model in the DET system is modeled using empirical data. The frequency responses in Figs.7.5 and 7.6 using these fitted models again match very well with the exact model.

\subsection{Conclusions}

In this chapter, empirical data modeling and analysis of power systems is discussed. The analytical transfer function model of a one-port and two-port black box can be developed based on the measured frequency response of port characteristics.

A reduced order modeling technique is also implemented using the complex curve fitting method. This technique allows the elimination of non-dominating poles and zeros as well as poles and zeros in close proximity, which can be ignored for all practical purposes. The reduced order model greatly facilitates analysis and trouble-shooting of complex spacecraft power system and with much improved computational efficiency. 


\section{Chapter 8}

\section{CONCLUSIONS}

The principal objective of this dissertation is the development of a comprehensive large scale power system modeling that facilitates the design and analysis of present and future spacecraft power systems. Methodologies of model building and analysis of large scale power system are presented.

The principal goal is accomplished by utilizing the modular concept such that the total system is configured by interconnecting individual component modules. The hybrid g-parameter two-port subsystem coupling method is employed as the standard interconnection law. This provides bi-directional information flow among components so that the maximum flexibility can be achieved in system model building. The flexibility in model building gives more than convenience but the success of the system level modeling depends largely on it due to the difficulties involved in large scale system modeling as stated in Chapter 1 . The modular approach also allows the system performance parameters to be expressed as both analytically and empirically measurable quantities. Thus, the model can be verified at various component levels. Also, since the system performance parameters such as stability, audiosusceptibility, impedance characteristics, etc. are derived with a focus on either local or global behavior, the model allows one to predict and trouble-shoot the undesired interactions that often occur among various subsystems. 
The success of the system level model building reduces the uncertainties in the model to the component level which implies the accuracy of the system model depends now on that of component models. As stated in Chapter 1, behaviors of individual components of spacecraft power system are mostly well understood and new and more powerful techniques for component level modeling are currently under development. In this study, existing component modeling techniques are adapted to develop unterminated component models (two-input, two-output). Each model consists of large signal time-domain model and small signal model. The large signal model simulates the actual behavior of the system including various nonlinearities in the practical circuit. The small signal model characterizes dynamics of the system about a steady state operating condition. The integration of the component models is done by the host software system, EASY5, which was identified from the preliminary study of applicable existing software systems, as the most suitable general purpose program. It provides maximum flexibility in component model building such that each component can be modeled with state differential or difference equations, transfer functions, empirical data and combination of these.

The comprehensive analysis of a spacecraft power system, the secondary goal of the study, is performed using the Direct Energy Transfer (DET) power system as an example. Also, the large signal behavior of the general solar array spacecraft power system is analyzed.

The nonlinear solar array output characteristic coupled with a constant power load is shown to result in multiple equilibrium points. The state plane analysis shows that the equilibrium points in the region where 
the solar array behaves as a constant current source are unstable. There exists a separatrix in this region which separates the state plane trajectories into two distinct patterns that ultimately lead to two quite discernible operating points. However, only one, corresponding to the solar array constant voltage source region, is the desirable system operating point. Thus, the system's initial start-up condition must be set in such a way that it leads to the desired operating point.

The behavior of the operating point near the solar array maximum power point is analyzed. Regarding stability and transient response, system operation in this region is less commendable than in the region where the solar array behaves as a stiffer voltage source. One way of improving dynamics of a system that utilizes the solar array maximum available power is to dissipate some power in a shunt circuit. This makes the source characteristic a stiff voltage source.

Since the battery discharger in the DET system operates over a range from a no-load condition through the discontinuous conduction mode (DCM), to the continuous conduction mode (CCM), the feedback compensator of the battery discharger regulator must be designed carefully to ensure the stability for all conditions. A proportional gain control is most appropriate for a converter operating in DCM to speed up the system response. In CCM, a phase lead type compensator is required to compensate the excessive phase lag from the complex pole. Thus, integrator is not suitable for a compensator in the battery discharger regulator because of the power stage open loop pole near the origin. 
One of the major difficulties in large scale power system analysis is the subsystem interaction problem. The unterminated two-port component modeling allows one to investigate the interactions at the component, subsystem and system level viewed from either the source or the load.

For a spacecraft power system that uses many switching regulators connected in parallel on a common bus, such as in the DET system, interactions should be examined both at the local level and the system level. Analysis shows that as the number of regulators increases, the margin of stability of the system tends to diminish due to the parallel interactions.

As stated in Chapter 7, due to the lack of an assessment of certain parameter values in some spacecraft component models, experimentation is . the only effective means of obtaining models with acceptable accuracy. Employing the complex curve fitting technique, the analytical transfer function model of a one-port and two-port black box is developed based on the measured frequency response of the port characteristics. If the fitted model reasonably matches (over the frequency range of interest) the frequency response of the black box, the two subsystems are expected to be dynamically equivalent. This is verified by performing a system level analysis using both the fitted model and the actual model for a subsystem.

A reduced order modeling technique is also implemented using the complex curve fitting method. This technique enables one to eliminate nondominating poles and zeros as well as poles and zeros in close proximity which can be ignored for all practical purposes. The reduced order model 
greatly facilitates the analysis and trouble-shooting of a complex spacecraft power system with much improved computational efficiency. 


\section{REFERENCES}

1. J. Cassineli, et al., "Analytical Modeling of Spacecraft Power System," Final Report to NASA GSFC, 1982, TRW, Redondo Beach, CA

2. D. L. Sommer, I. S. Mehdi, "High Voltage DC Electrical System Stability Analysis," Final Report to Naval Air Development Center, Oct. 1979, Boeing Military Airplane Company, Seattle, WA

3. R. D. Middlebrook, S. Cuk, "A Generalized Unified Approach to Modeling Switching Converter Power Stages," IEEE PESC 1976

4. D. J. Short, "An Improved Switching Regulator Power Stage Model using Discrete-Average Techniques," Ph.D. Dissertation, 1982, VPI\&SU

5. D. J. Packard, "Discrete Modeling and Analys is of Switching Regulators," Ph.D Theis, CIT Pasadena Calif., May 1976

6. B. H. Cho, F. C. Lee, "Feasibility Study of Analytical Modeling of Spacecraft Power System," Final Report to Naval Research Lab., Aug. 1983 , VPI\&SU

7. J. M. Voss, J. G. Gray, "A Regulated Solar Array Model - A Tool For Power System Analysis," IEEE PCSC 1970 pp.12-17

8. A. Capel, et al., "Power System Simulation of Low Orbit Spacecraft: The EBLOS Computer Program," IEEE PESC 1982, pp.272-285

9. R. D. Middlebrook, S. G. Kimble, "Design of the HEAO Main Bus Shunt Regulator," IEEE Trans. AES, March 1976

10. W. Burns, III, et al., "A Digital Simulation and Study of Direct Energy Transfer Power Conditioning System," IEEE PESC, 1975 pp.138-149

11. D. K. Decker, "Methods for Utilizing Maximum Power from a Solar Array," JPL Quarterly Technical Review, April 1972, pp.37-47

12. E. N. Costogue, S. Lindena, "Comparison of Candidate Solar Array Maximu Power Utilization Approaches," IECEC 1976, pp.1449-1456

13. R. D. Middlebrook, "Input Filter Considerations in Design and application of Switching Regulator," IEEE IAS 1976

14. E. C. Levy, "Complex-Curve Fitting," IRE Trans. Auto. Cont., May 1959

15. C. Sanathanan, J. Koerner, "Transfer Function Synthesis as a Ratio of Two Polynomials," IEEE Tran. on Auto. Cont., Jan. 1963, pp.56-58 
16. R. Seidal, "Transfer Function Parameter Estimation from Frequency Response Data," NASA tm x3286, 1975

17. R. Fletcher, C. M. Reeves, "Function Minimization by Conjugate Gradients," Computer J., vol. 7, No. 2, July 1964, pp.149-154

18. D. Zerbel, D. Decker, "AC impedance of Silicon Solar Cells," IECEC, 1970

19. P. Chen, et al., "Experimental Determination of Series Resistance of $\mathrm{p}-\mathrm{n}$ Junction Diodes in Solar Cells," IEEE Tran. on Elec. Devices, vol.ED-25, march 1978 , pp.386-388

20. C. Fang, J. Hanser, "Comparison of Theoretical and Experimental Solar Cell Performance," IECEC 1978

21. P. Chetty, et al., "Microprocessor Controlled Digital Shunt Regulator," IEEE Tran. on AES, March 1980, pp.191-200

22. F. C. Lee, et al., "Generalized computer Aided Discrete Time Domain Modeling Analysis of DC-DC Converters," IEEE PESC 1977

23. F. C. Lee, et al., "Modeling of Switching Regulator Power Stage with or without Zero-Inductor-Current Dwell Time," IEEE IECI Trans. Aug. 1979

24. W. M. Rintala, et al., "Intelsat V Power Control Electronics System," IECEC 1979, pp.1349-1349

25. E. Doebelin, System Modeling and Response, John Wiley \& Sons, 1980 


\section{The vita has been removed from the scanned document}

